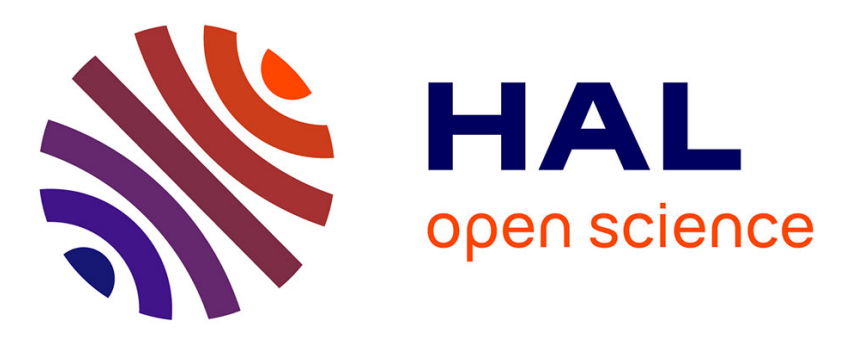

\title{
Le réveil des classes moyennes ivoiriennes? Identification, caractérisation et implications pour les politiques publiques
}

Jean-Philippe Berrou, Dominique Darbon, Christian Bouquet, Anne Bekelynck, Matthieu Clément, François Combarnous, Eric Rougier

\section{To cite this version:}

Jean-Philippe Berrou, Dominique Darbon, Christian Bouquet, Anne Bekelynck, Matthieu Clément, et al.. Le réveil des classes moyennes ivoiriennes? Identification, caractérisation et implications pour les politiques publiques. [Rapport de recherche] AFD - Agence Française de Développement. 2018, pp.2018 - 71. hal-02147840

\author{
HAL Id: hal-02147840 \\ https://hal.science/hal-02147840
}

Submitted on 6 Sep 2019

HAL is a multi-disciplinary open access archive for the deposit and dissemination of scientific research documents, whether they are published or not. The documents may come from teaching and research institutions in France or abroad, or from public or private research centers.
L'archive ouverte pluridisciplinaire HAL, est destinée au dépôt et à la diffusion de documents scientifiques de niveau recherche, publiés ou non, émanant des établissements d'enseignement et de recherche français ou étrangers, des laboratoires publics ou privés. 


\section{Papiers de Recherche | Research Papers}

\section{Le réveil des classes moyennes ivoiniennes? Identific ation, caractérisation et implic ations pour les politiques publiques}

J ean-Philippe BERROU*

Dominique DARBON ${ }^{\ddagger}$

Christia n BOUQ UET ${ }^{*}$

Anne BEKELYNCKł‡

\author{
Matthieu CLÉMENT \\ Franç ois COMBARNOUS§ \\ Éric ROUGIER ${ }^{+\dagger}$
}

J uillet 2018

Pourciter ce papier:

Contact à l'AFD :
BERROU, J.-P., D. DARBO N, C. BOUQUET, A. BEKELYNCK, M. CLEMENT, F. COMBARNOUS et E. ROUGIER (2017), “Le réveil des classes moyennes ivoiriennes? Identification, caractérisation et implications pour les politiques publiques ", Papiers de rec herc he AFD, $n^{\circ} 2018-71$, J uillet.

Anda David (davida@afd.fr)

LAM CNRS, Sciences Po Bordeaux GREThA C NRS, Université de Bordeaux LAM CNRS, Sciences Po Bordeaux GREThA CNRS, Université de Bordeaux LAM CNRS, Sciences Po Bordea ux

GREThA CNRS, Université de Bordeaux

PAC-CI, ANRSCôte d'Ivoire

Avec la participation et l'appui technique de l'École Nationale Supérieure de Statistique et d'Ec onomie Appliquée - ENSEA (Abidjan) 


\section{Papiers de Recherche de l'AFD}

Les Papiers de Recherche de l'AFD ont pour but de diffuser rapidement les résultats de travaux en cours. Ils s'adressent principalement aux chercheurs, aux étudiants et au monde académique. Ils couvrent l'ensemble des sujets de travail de l'AFD : a nalyse économique, théorie économique, a nalyse des politiques publiques, sciences de l'ingénieur, sociologie, géographie et anthropologie. Une public ation dans les Papiers de Recherche de l'AFD n'en exc lut auc une autre.

L'Agence Française de Développement (AFD), institution financière publique qui met en œuvre la politique définie par le gouvemement français, agit pour combattre la pauvreté et favoriser le développement durable. Présente sur quatre continents à travers un réseau de 72 bureaux, l'AFD finance et accompagne des projets qui améliorent les conditions de vie des populations, soutiennent la croissance économique et protègent la planète. En 2014, l'AFD a consacré 8,1 milliards d'euros au financement de projets dans les pays en développement et en faveur des Outre-mer.

Les opinions exprimées dans ce papier sont celles de son (ses) auteur(s) et ne reflètent pas nécessairement celles de l'AFD. Ce document est publié sous l'entière responsabilité de son (ses) auteur(s).

Les Papiers de Recherche sont téléchargeables sur: https://www.afd.fr/fr/ressources

\section{AFD Research Papers}

AFD Research Papers are intended to rapidly disseminate findings of ongoing work and mainly target researchers, students and the wider academic community. They cover the full range of AFD work, including: economic analysis, economic theory, policy analysis, engineering sciences, sociology, geography and anthropology. AFD Research Papers and other public ations a re not mutually exc lusive.

Agence Française de Développement (AFD), a public financial institution that implements the policy defined by the French Govemment, works to combat poverty and promote susta inable development. AFD operates on four continents via a network of 72 offices and finances and supports projects that improve living conditions for populations, boost economic growth and protect the planet. In 2014, AFD earmarked EUR 8.1bn to finance projects in developing countries and for overseas France.

The opinions expressed in this paper are those of the author(s) and do not necessarily reflect the position of AFD. It is therefore published under the sole responsibility of its author(s).

AFD Research Papers can be downloaded from: https://www.afd.fr/en/ressources

AFD, 5 rue Roland Barthes

75598 Paris Cedex 12, France

ResearchPapers@afd.fr

ISSN 2492 - 2846 


\section{Le réveil des classes moyennes ivoiriennes? \\ Identification, caractérisation et implications pour les politiques publiques}

Jean-Philippe Berrou, LAM CNRS, Sciences Po Bordeaux

Dominique Darbon, LAM CNRS, Sciences Po Bordeaux

Anne Bekelynck, PAC-CI, ANRS Côte d'Ivoire

Christian Bouquet, LAM CNRS, Sciences Po Bordeaux

Matthieu Clément, GREThA CNRS, Université de Bordeaux

François Combarnous, GREThA CNRS, Université de Bordeaux

Éric Rougier, GREThA CNRS, Université de Bordeaux

\section{Résumé}

Le cas de la Côte d'Ivoire présente une historicité de longue durée du processus de différenciation sociale voire d'émergence de catégories « moyennes ». Dans ce papier, à partir de l'analyse de la distribution des revenus des ménages ivoiriens en 2015, la classe intermédiaire de revenu est isolée sur la base d'un intervalle mixte combinant une borne inférieure absolue, fixée à 4 dollars par jour et par tête, et une borne supérieure relative, fixée au $95^{\mathrm{e}}$ percentile de la distribution. Ainsi calibrée, la classe moyenne monétaire ivoirienne, «les gens du milieu », représente finalement une masse relativement limitée de 26,4\% de la population. 48,1\% des chefs de ménage de cette catégorie est sans éducation et, si elle est majoritairement urbaine (à près de $60 \%$ ), seul $16 \%$ de cette classe moyenne est abidjanaise. Un fort dualisme de cette classe moyenne se dégage avec, d'un côté, en minorité (21\%), une strate haute et stabilisée constituée plutôt d'héritiers des groupes intermédiaires des années 1960-1970 et, de l'autre, une strate basse majoritaire $(79 \%)$ composée de ménages en situation de petite prospérité et de grande vulnérabilité et composés d'indépendants informels urbains et d'agriculteurs. Enfin, les classes moyennes ivoiriennes sont très peu engagées politiquement. Elles peuvent d'autant moins s'exprimer que leur forte hétérogénéité interne et leur faible expérience de mobilisation collective réduisent fortement leur capacité de faire pression sur le politique, sauf pour des groupes qui interviennent alors plus sur la base de leur identité socio professionnelle.

Mots-clés : Classe-moyenne, Politique publique, Côte d'Ivoire, Mobilité sociale

Classification JEL : I3, D31, O2

Version originale : Français

Acceptée : $\quad$ Juillet 2018 


\section{Introduction}

L'émergence des « classes moyennes » ${ }^{1}$ en Côte d'Ivoire présente des caractéristiques historiques, sociologiques et politiques très particulières pour le continent africain. Inscrit dans la longue durée, le processus ivoirien de différenciation des stratifications sociales est marqué par des temporalités et des mécanismes d'accumulation spécifiques propres au pays : ancienneté du bouleversement de la structure sociale et des formes de transmission intergénérationnelle des positions sociales depuis la colonisation, diversité des configurations économiques d'une économie de plantation et instabilité du régime de croissance (du miracle ivoirien des années 1960 et 1970 à celui des années 2010 en passant par 30 ans d'instabilités socioéconomiques), complexité des modes de régulation politique (du parti unique jusqu'en 1990 à un multipartisme stabilisé sur la période récente, en passant par une guerre des héritiers dans les années 90, la question de l'ivoirité, la crise militaro politique et la partition du pays dans les années 2000). Audelà du narratif de la " manufactured controversy » commun à toutes les sociétés africaines (Darbon, 2018 ; Melber, 2016), le cas ivoirien interroge de manière exemplaire la question de la réalité de l'émergence de classes moyennes et impose donc de resituer l'analyse dans le temps long des régimes de croissance et des compromis sociopolitique du pays et de travailler aussi bien en termes de flux que de stocks (de néo classes moyennes accédant à ce statut que d'héritiers de processus intergénérationnels), en identifiant les mécanismes d'accumulation qui portent les modifications des statuts sociaux.

La Côte d'Ivoire est un pays africain de taille moyenne, qui n'est pas enclavé, et qui s'étend du sud au nord entre les 4ème et 10ème parallèles. Située en Afrique de l'Ouest, le pays est bordé par le Mali, le Burkina Faso, le Libéria, la Guinée et le Ghana. Devenue colonie française en 1893, elle est libérée du joug colonial en 1960 sous l'égide de Félix Houphouët-Boigny (1905-1993). La Côte d'Ivoire s'est ensuite imposée comme un leader historique de la sous-région: son dynamisme a longtemps contrasté avec les difficultés rencontrées par ses voisins avant que le pays ne connaisse lui-même une longue période de crise sociale, économique et politique.

L'économie ivoirienne est caractérisée par l'importance de son secteur agricole, à la fois en termes de production (plus de $25 \%$ du PIB en moyenne depuis 1960) et surtout d'emploi (près de la moitié de la population). Aujourd'hui l'agriculture contribue à $22 \%$ du PIB, derrière le secteur des services $(46 \%)$ et de l'industrie (33\%). La trajectoire socioéconomique du pays a été et reste encore profondément marquée par l'évolution et les modalités de gestion et de redistribution de sa « rente forêt » (du bois au binôme cacao-café) (Cogneau et Mesplé-Somps, 2002). Fortement extravertie sur le plan économique, notamment du fait de sa forte dépendance aux exportations agricoles, la Côte d'Ivoire l'est aussi sur le plan démographique. Peuplée d’à peine 3,5 millions d'habitants en 1960, la Côte d'Ivoire a franchi le palier des 10 millions en 1988, puis des 15 millions en 1998. Aujourd'hui elle compte plus de 22 millions d'habitants, dont près de

1 Par mesure de facilité, nous utiliserons dans ce rapport le terme de "classes moyennes » comme synonyme de « catégories » ou " groupes intermédiaires de revenus » sans lui donner de signification sociale prédéfinie mais en retenant qu'il y a derrière cette étiquette bien plus qu'un phénomène statistique. 
5 millions d'étrangers (RGPH, 2014). En effet, son littoral maritime forestier initialement inhospitalier en raison du climat est demeuré longtemps sous-peuplé, au point que les colonisateurs ont dû organiser des transferts importants de populations venant de la zone soudano-sahélienne, davantage peuplée, vers cette zone forestière afin, dans un premier temps, d'exploiter le bois et de construire le chemin de fer reliant Abidjan à Ouagadougou puis, dans un second temps, de travailler dans les plantations de café et de cacao. Si la géographie humaine a donc été sensiblement modifiée au moment de la colonisation, ces mouvements de populations externes (arrivée massive de populations venant des pays voisins du nord) et internes (déplacement dus, entre autres causes, au développement des cultures de rente du café et du cacao) ont perduré après les indépendances (notamment durant la période du miracle ivoirien) (Bouquet, 2011). Longtemps supérieur à $3 \%$, le taux naturel d'accroissement reste donc élevé à 2,6\%. De ce fait, les jeunes de moins de 35 ans représentent $78 \%$ et les moins de 15 ans $42 \%$ du total. Le taux d'urbanisation qui était de $32 \%$ en 1975 a franchi la barre des $50 \%$ lors du dernier recensement de 2014 (RGPH, 2014).

L'histoire récente du peuplement est donc singulière, les brassages et les mélanges auraient pu constituer un avantage par rapport aux replis identitaires que connaissent nombre de pays africains. On a pu le croire tant que le pays a été dirigé par Félix Houphouët-Boigny dont les choix économiques et compromis sociopolitiques ont permis de faire cohabiter les communautés autochtones, allochtones et allogènes. Avec un itinéraire peu banal, ce fis de chef coutumier, militant fondateur (dès 1932) et leader du plus grand syndicat des planteurs, puis du Parti Démocratique de Côte d'Ivoire (PDCI), a connu (et généré ?) durant les deux premières décennies de son mandat l'euphorie socioéconomique du modèle ivoirien ${ }^{2}$. C'est d'ailleurs dans ce contexte que les cultivateurs de café et de cacao ont pu constituer, autour des années 1960 et après, une catégorie sociale assimilable à une classe moyenne.

Ce succès a commencé à s'effriter à partir des années 1980 lorsque, à l'instar de nombreux pays en développement, la Côte d'Ivoire connait une sévère crise économique et financière (crise de la dette extérieure puis période d'ajustements structurels). Plus encore, peut être faute d'avoir su désigner clairement son successeur avant de mourir en 1993, le pays traverse ensuite une crise de succession opposant notamment Henri Konan Bédié, qui en tant que président de l'Assemblée nationale succède à Houphouët Boigny, et Alassane Ouattara, le Premier ministre qui jouissait pourtant de la confiance du « vieux ». Dès lors, la cohésion nationale que le vieux leader avait réussi à maintenir va commencer à se lézarder et le pays va connaitre de longues années d'instabilités politiques, sociales et économiques. À partir de 1996, l'émergence du concept d'« ivoirité », qui distingue les «Ivoiriens de souche multiséculaire » des autres - c'est-à-dire essentiellement les populations du nord assimilées à des étrangers, génériquement nommées

2 La trajectoire politique de Félix Houphouët-Boigny est en effet originale: un temps proche du parti communiste français dans le cadre du RDA (Rassemblement démocratique africain, fâttière du Parti démocratique de Côte d'Ivoire qu'il avait créé en 1946), il a effectué une carrière ministérielle française si proche du général de GAULLE qu'il est allé jusqu'à défendre l'Algérie française et à résister à l'idée des indépendances africaines. Après son accession au pouvoir suprême en 1960, il fut l'un des rares parmi les nouveaux chefs d'État africains à ne pas choisir l'alignement sur le modèle soviétique, promettant même à son voisin Kwamé NKRUMAH qu'un jour la Côte d'Ivoire dépasserait le Ghana. 
« dioulas »- met à mal la culture du vivre ensemble. La montée des tensions conduit au coup d'État du général Robert Gueï en décembre 1999, événement par lequel la Côte d'Ivoire retombe dans la catégorie des pays africains ordinaires où l'on chasse du pouvoir par la force un président élu démocratiquement. Une modification constitutionnelle permet ensuite d'écarter Alassane Ouattara de la candidature à l'élection présidentielle suivante, et c'est l'opposant historique Laurent Gbagbo, fondateur du FPI (Front populaire ivoirien) qui accède à la magistrature suprême en octobre 2000, dans des conditions qu'il qualifiera lui-même de "calamiteuses ». Reprenant à son compte la doctrine ivoiritaire, il est immédiatement contesté, voire menacé dans sa fonction puisqu'une tentative de coup d'État aboutit en septembre 2002 à la partition du pays en deux moitiés, dont la partie nord restera contrôlée pendant près de cinq années par les « rebelles » dirigés par l'ex-leader étudiant Guillaume Soro. Il faudra attendre dix ans pour que soit organisée une nouvelle élection présidentielle, au cours de laquelle l'accord de 2005 entre les deux anciens frères ennemis du RDR (Ouattara) et du PDCI (Bédié) pour créer l'alliance RHDP (Rassemblement des Houphouëtistes pour la Démocratie et la Paix) permet au premier de remporter la victoire avec $54 \%$ des suffrages exprimés au second tour (28 novembre 2010). On pense alors que la sortie du tunnel est proche, mais la contestation par Laurent Gbagbo des résultats du scrutin déclenche une violente crise postélectorale qui ne s'achève que le 11 avril 2011 par la reddition de l'ex-chef de l'État et un bilan de plus de 3000 victimes.

La Côte d'Ivoire a depuis lors peu à peu retrouvé une certaine sérénité et une orientation économique claire. Malgré un environnement international incertain, le pays a renoué avec une croissance économique spectaculaire (en moyenne supérieure à $8 \%$ par an) portée par le secteur agricole, des plans d'investissement ambitieux et une amélioration du climat des affaires. En octobre 2015, Alassane Ouattara est réélu pour un second mandat et, fort d'une majorité RDR au Parlement, il peut espérer poursuivre ses chantiers visant à faire de la Côte d'Ivoire un pays "émergent » en 2020. Mais, au début de 2017, il est confronté à une triple crise : socioéconomique avec les grognes des planteurs de cacao, face à la chute des prix, et des fonctionnaires qui réclament le versement des arriérés de salaire qui leur ont été promis ; politique avec l'éclatement de l'alliance RHDP et la reconstitution de groupes parlementaires séparés ; et militaire, avec les ex-rebelles qui se mutinent dans l'armée et réclament des primes exorbitantes.

C'est dans ce contexte que s'inscrivent les enjeux relatifs à l'émergence ou la réémergence de ce qui est appelé par certains acteurs (magazines économiques, études de marché ou rapports d'organismes internationaux) de manière aussi contestée que contestable la ou les classes moyennes ivoiriennes. L'essor de ces classes moyennes est souvent présenté dans la littérature comme déterminant dans le processus de développement et d'émergence. Il permet l'accroissement des dépenses de consommation et l'expansion du marché domestique et, de fait, favorise la croissance du PIB. Il contribue à la diversification de la demande sociale et de la consommation de biens et services (Banerjee et Duflo, 2008). Il diversifie la structure sociale et révèle le potentiel de mobilité ascendante entre les strates de la société. Il redistribue enfin les enjeux politiques, en rendant possible à la fois une stabilisation politique accrue autour d'une masse importante de la population et la possibilité d'alliances alternatives favorisant le contrôle 
du pouvoir et l'inclusion politique (Lipset, 1959 ; Easterly, 2001; Loayza et al., 2012), ou en diversifiant et renforçant les sources de conflits et de revendication au risque de favoriser une déstabilisation (Huntington, 1968 ; Villegas, 2012). On appelle ainsi émergence des classes moyennes un mouvement indifférencié de sortie de pauvreté à la marge et d'enrichissement venant briser l'opposition classique dans la plupart des sociétés africaines entre riches et pauvres. Ce mouvement n'est ni général dans tous les pays, ni homogène sur chaque territoire national et peut donner lieu à des interprétations très différentes allant de l'émergence à la constance de la pauvreté massive en passant par la recomposition de la structure sociale matérialisée par des niveaux différenciés d'accès à la prospérité (Darbon et Toulabor, 2014).

Dans le cas de la Côte d'Ivoire, la question de l'essor de ces classes moyennes est d'autant plus complexe que le pays accumule des situations associant continuité historique du mouvement de transformation de la stratification sociale au profit de catégories intermédiaires et rupture de la linéarité du processus : du miracle économique des années 1960-1970 avec l'émergence avant la lettre d'une grande et d'une petite bourgeoisie d'Etat et d'entrepreneurs, aux crises politico économiques à partir des années 1980 générant sur 30 ans une réduction de moitié de son PIB par habitant. La Côte d'Ivoire est depuis lors en quête d'un retour sur le trend des années 1960. Elle est sur la période récente perçue par les investisseurs comme l'un des meilleurs risques du continent, vantée notamment pour la puissance et la qualité de sa classe moyenne abidjanaise (PwC, 2015). La Côte d'Ivoire présente ainsi tous les paradoxes voire les apories qui caractérisent le discours sur les classes moyennes africaines ce qui permet à la fois d'en retirer la part de mythologie et d'inexactitudes tout en essayant de dévoiler les dynamiques sociales à l'œuvre et de comprendre les enjeux en cours.

La question de l'essor des classes moyennes en Côte d'Ivoire, en plus d'être particulièrement d'actualité, intéresse donc tout autant la communauté scientifique que les décideurs politiques. Analyser comment se compose cette catégorie intermédiaire de la société, quels sont ses comportements, attentes et aspirations notamment en matière de consommation mais aussi comprendre comment le développement supposé de ce groupe influence le design des politiques publiques sont les questions auxquelles cette étude se propose de répondre. Partant de là, celle-ci poursuit quatre objectifs principaux : (i) proposer une synthèse de la littérature sur les classes moyennes ivoiriennes en lien avec la trajectoire socioéconomique et politique du pays ; (ii) proposer une méthode d'identification des classes moyennes ivoiriennes permettant notamment d'en souligner l'hétérogénéité ; (iii) analyser les comportements et aspirations de ces classes moyennes et (iv) évaluer les implications de l'expansion des classes moyennes en matière de politiques publiques et inversement l'impact éventuel des politiques publiques sur leur émergence et leur configuration.

D’un point de vue méthodologique, nous adoptons une démarche séquentielle combinant analyse de la littérature académique et institutionnelle et méthodologies quantitatives et qualitatives. Dans un premier temps, nous proposons une analyse d'économie politique, institutionnaliste et historique des processus de stratifications sociales et de formation des classes moyennes dans le pays au regard des régimes de croissance dans la longue période. Dans un second temps, nous 
mettons en œuvre une analyse quantitative basée sur les données de l'enquête ENV 2015 (Enquête Niveau de Vie des ménages) collectées par l'INS (Institut National de la Statistique) afin d'identifier les classes moyennes ivoiriennes contemporaine dans toute leur hétérogénéité. Ensuite, les données quantitatives de l'ENV et les données collectées par une enquête qualitative auprès des ménages des classes moyennes permettent de les caractériser plus finement, de comprendre leurs dynamiques et de mettre en évidence leurs principales aspirations, notamment en matière de politiques publiques. Dans un dernier temps, l'analyse de la littérature institutionnelle et les résultats d'une enquête qualitative auprès d'institutions représentatives des domaines clés des politiques publiques permettent d'évaluer la contribution de ces politiques à la montée en puissance des classes moyennes et comment, en retour, ces classes moyennes peuvent en influencer l'orientation et le contenu.

Cette étude se structure de la façon suivante. La première partie dresse un état des lieux de la littérature sur les classes moyennes en Côte d'Ivoire en recadrant les principales évolutions de la stratification sociale dans la trajectoire économique du pays (régime de croissance de long terme). La deuxième partie est consacrée à l'identification et à la caractérisation des classes moyennes ivoiriennes et se propose d'exposer les principaux résultats issus de l'analyse quantitative et de l'enquête qualitative auprès des ménages. Dans la troisième partie, nous nous focalisons sur les implications de l'essor des classes moyennes sur les politiques publiques en mobilisant notamment les résultats de l'enquête qualitative auprès des institutions.

\section{Les instabilités du régime de croissance et les processus de différenciations sociales en Côte d'Ivoire : les classes moyennes dans la tourment}

L'émergence des classes moyennes en Côte d'Ivoire s'inscrit dans le temps long des instabilités du régime de croissance. Du miracle ivoirien des «vingt glorieuses » aux incertitudes de la période dite de la «conjoncture», en passant par des épisodes de reprise jusqu'au renouveau contemporain de la croissance, ces différentes phases de croissance ont accompagné les processus de stratification sociale selon des modalités spécifiques et non continues. Aujourd'hui, alors que la Côte d'Ivoire renoue avec un cycle de forte croissance économique, les enjeux relatifs aux classes moyennes sont autant sociaux (cohésion sociale dans un contexte de réconciliation nationale), économiques (développement d’un marché interne nécessaire aux objectifs de diversification de l'économie) que politique (légitimité et stabilité politique dans un contexte de fortes attentes sociales). La mise en perspective historique des régimes de croissance en Côte d'Ivoire (1.), tant sur le plan de la description des caractéristiques des différentes phases de croissance que de l'analyse de leurs déterminants économiques et politiques fondamentaux, permettra de mieux saisir les discontinuités et instabilités des processus de stratifications sociales au sein desquelles peut être analysée la formation de ces catégories intermédiaires (2.). 


\subsection{Une mise en perspective historique des régimes de croissance en Côte d'Ivoire}

Depuis 2011, la Côte d'Ivoire connaît un renouveau économique suscitant tant l'attrait des investisseurs privés que l'enthousiasme des institutions internationales. Il s'agit ici de revenir brièvement sur les caractéristiques de ce succès économique et ses enjeux (notamment sociaux) (1.1.) avant d'en faire l'analyse plus précise, tant en le resituant dans le temps long des différentes phases de croissance (1.2.) qu'en identifiant les transformations structurelles réellement à l'œuvre au regard des déterminants fondamentaux du modèle de croissance ivoirien (1.3.).

\subsubsection{Un nouveau miracle économique ivoirien (2011-)}

Malgré un environnement international incertain, depuis 2011 et la fin des violences postélectorales la Côte d'Ivoire renoue avec un cycle de forte croissance économique. Cette croissance se fait à un rythme particulièrement élevé : après une chute du PIB de 4,1\% en 2011, celui-ci-bondit de 9,2\% en 2012 et continue à évoluer sur un rythme de croissance moyen avoisinant les 8-9\% par an jusqu'en 2016 (Banque Mondiale, 2017 ; OCDE, 2016). A tel point que sur cette dernière année, la Côte d'Ivoire devient le deuxième pays du continent africain au taux de croissance le plus élevé, derrière l'Ethiopie (Graphique nº1). Malgré un ralentissement récent, les perspectives des institutions internationales restent bonnes, la Banque Mondiale estimant que le taux de croissance du PIB devrait converger autour de 7,5\% en 2019.

Du côté de la demande les moteurs de ce renouveau de la croissance ont progressivement évolué sur la période. Dans l'ensemble, c'est la demande intérieure qui joue un rôle central. Du côté de l'investissement, de 13,1\% du PIB en 2012, la FBCF passe à 19\% du PIB en 2013 et se maintient jusqu'en 2016 autour de 17\% du PIB (Banque Mondiale, 2017 ; OCDE, 2016). L'investissement public tient ici une place prépondérante dans un contexte de reconstruction des infrastructures dégradées après plusieurs années de crises et de violences. Les plans nationaux de développement (PND) 2012-2015 et 2016-2020 attestent de programmes d'investissement ambitieux. Dans un climat politique plus apaisé l'investissement privé a répondu à la dynamique de l'investissement public et a lui aussi nettement augmenté. Il est ainsi attendu qu'il prenne progressivement plus significativement le relais de l'investissement public. Dans ce contexte politique plus stable, la consommation des ménages joue elle aussi un rôle moteur sous l'impulsion d'une augmentation des revenus soutenue notamment par plusieurs mesures politiques (revalorisation du salaire des fonctionnaires, accroissement significatif du SMIG, réforme du secteur cacao). Cette expansion de la consommation pourrait certainement préfigurer la consolidation d'une classe moyenne si elle concernait une plus grande partie de la population et pas seulement celle relevant des emplois salariés du secteur officiel public et privé. Du côté de l'offre, les principaux secteurs d'activité du pays ont contribué à cette croissance. L'agriculture, vivrière (riz, plantain, maïs, manioc) comme d'exportation (cacao, café, noix de cajou, caoutchouc, huile de palme), a connu une progression particulièrement forte portée par des prix internationaux incitatifs (notamment les prix du cacao) et des récoltes en hausse à la faveur de bonnes conditions climatiques (le retournement de ces deux facteurs pour certaines productions agricoles expliquant le ralentissement récent de la croissance). Le secteur secondaire a été un bénéficiaire assez direct des grands programmes 
d'investissement publics à travers les activités de constructions (autoroute entre Abidjan et Yamoussoukro, hôpital Moscati, et troisième pont Marcory-Riviera). Le secteur des services, qui représente le secteur le plus important de l'économie (50\% du PIB), a joué un rôle moteur au travers de la dynamique du secteur des télécommunications (réorganisation du secteur de la téléphonie mobile), de l'énergie (effort d'extension de la distribution d'électricité dans le pays), du commerce et des transports.

\section{Graphique $\mathrm{n}^{\circ} 1$ - Le second pays avec la croissance économique la plus rapide sur le continent africain en 2016}
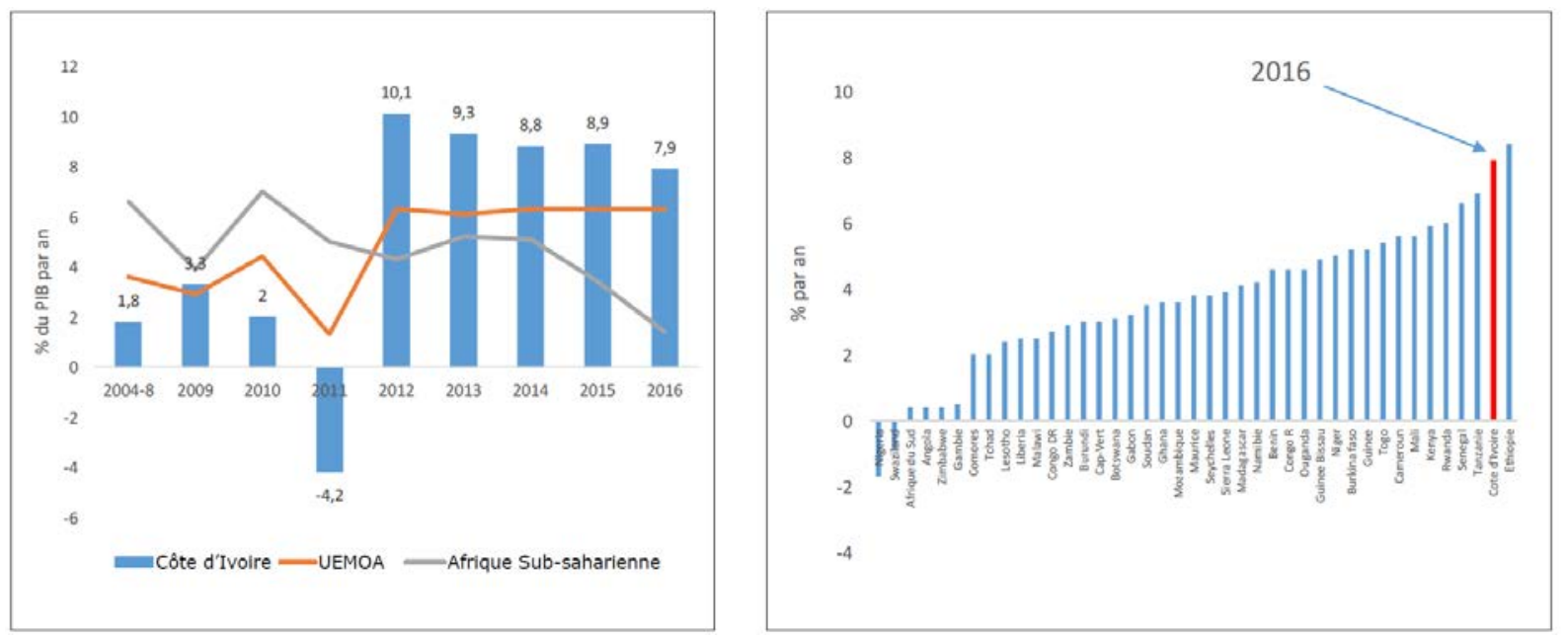

Source: Banque Mondiale (2017, p. 2)

Ces dynamiques s'inscrivent dans un climat des affaires et un environnement macroéconomique favorables. Le rapport Doing Business de 2017 classe ainsi la Côte d'Ivoire à la 2ème place au niveau ouest africain derrière le Ghana (120e). Le pays gagne aussi 14 places à l'Indice MoIbrahim sur la gouvernance Africaine entre 2014 et 2015 et se classe au 21ème rang sur 54 (AEO, 2017). Sur le plan macroéconomique, l'inflation reste stable en dessous des 3\% annuelle et la politique budgétaire relativement maitrisée grâce notamment à des efforts de recouvrement fiscaux. Le stock de la dette publique avoisine les $42 \%$ du PIB fin 2015, et le risque de surendettement sur la dette extérieure semble rester modéré (Banque Mondiale, 2017 ; AEO, 2017).

A l'instar de ce qui est parfois qualifié de « miracle » de la croissance en Afrique depuis le début des années 2000 (Young, 2012), ce renouveau de la croissance ivoirienne entraîne avec lui son lot d'espoirs en termes d'émergence du pays. Pour autant, les mêmes incertitudes qui alimentent le débat sur la situation du continent africain trouvent une résonance particulière dans le cas de la Côte d'Ivoire. Les progrès sur le plan de l'amélioration des conditions de vie, de la réduction de la pauvreté et des inégalités sont en effet très lents. Avec un faible nombre d'emplois créés dans les secteurs dits «modernes» (privés et publics), le marché de l'emploi en Côte d’Ivoire est dominé par le « secteur informel ». Selon les estimations les plus récentes, ce dernier représente autour de 90\% de l'emploi (AGEPE, 2014 ; INS, 2015) et contribue à hauteur de 30 à 40\% au PIB (FMI, 
2017). Dans ce contexte, il n'est pas étonnant que la situation sur le plan des conditions de vie (dans ses différentes dimensions, monétaires et non monétaires) ne se soit guère améliorée. Ainsi l'incidence de la pauvreté (la proportion d'individus sous le seuil de pauvreté), est de 46,3\% en 2015 (INS, 2015). Autrement dit près d'un ivoirien sur deux vit sous le seuil de pauvreté national (soit une dépense de consommation journalière inférieure à 737 FCFA). Cela ne représente qu'une baisse très légère par rapport à 2008 (48,9\%). Selon un sondage Gallup en 2013, à peine un tiers des ivoiriens se déclarent satisfait de leurs conditions de vie, le taux le plus faible parmi les pays du panel enquêté (graphique n²). De même, en 2014, l'enquête de la CAPEC révèle que la moitié des chefs de ménage interrogés considèrent que le niveau de vie de leur famille s'est dégradé depuis 2011 (CAPEC, 2014).

\section{Graphique $n^{\circ} 2$ - Satisfaction à l'égard des conditions de vie}

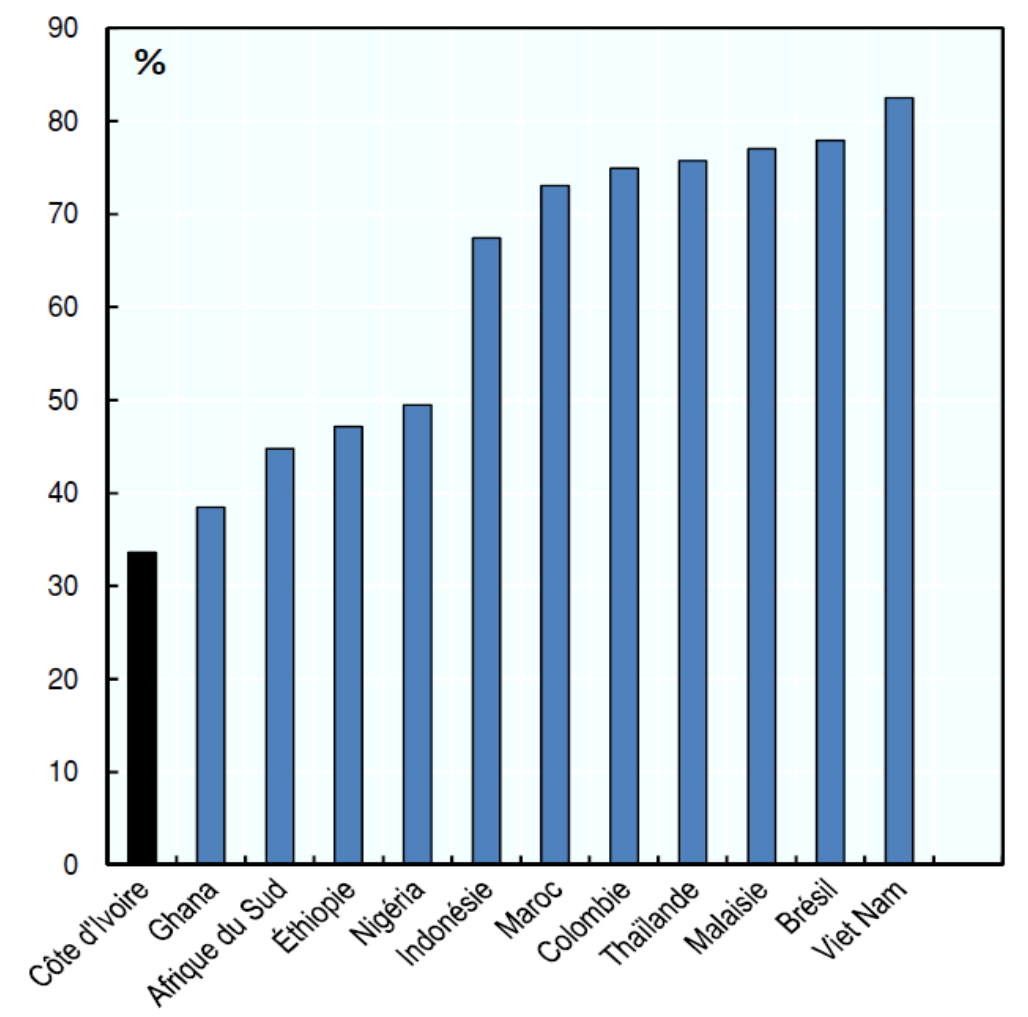

Note: Le graphique "Satisfaction à l'égard des conditions de vie » montre le pourcentage de personnes interrogées répondant « satisfaits » à la question "Êtes-vous satisfaits de votre niveau de vie?".

Source: Gallup 2013, in OCDE (2016a, p. 124)

Le défi d'une croissance inclusive permettant de réduire plus significativement la pauvreté et de constituer une large classe moyenne est ainsi un enjeu de taille pour la Côte d'Ivoire (Banque Mondiale, 2015; Ehrhart, 2015), en particulier dans le contexte récent de nouvelles tensions politiques. Dans ce cadre, il est nécessaire de mettre en perspective ce «nouveau miracle économique » ivoirien au regard des phases de croissance précédentes afin de distinguer dans cette période contemporaine ce qui relève de simples phénomènes de rattrapage ou d'effets 
conjoncturels exogènes de ce qui traduit de véritables transformations structurelles du modèle de croissance susceptibles d'assurer une plus large redistribution sociale.

\subsubsection{Retour sur les grandes phases du régime de croissance ivoirien depuis les indépendances}

Depuis son accession à l'indépendance jusqu'à 2011 la Côte d'Ivoire a connu une trajectoire de croissance particulièrement instable que l'on peut scinder en 4 grandes phases de durées différentes et qui se distinguent assez nettement au regard de l'évolution du taux de croissance du PIB par habitant (graphique $\left.n^{\circ} 3\right)^{3}$.

Graphique $n^{\circ} 3$ - PIB par tête et production agricole (cultures de rente) par tête $(1960-2010)$

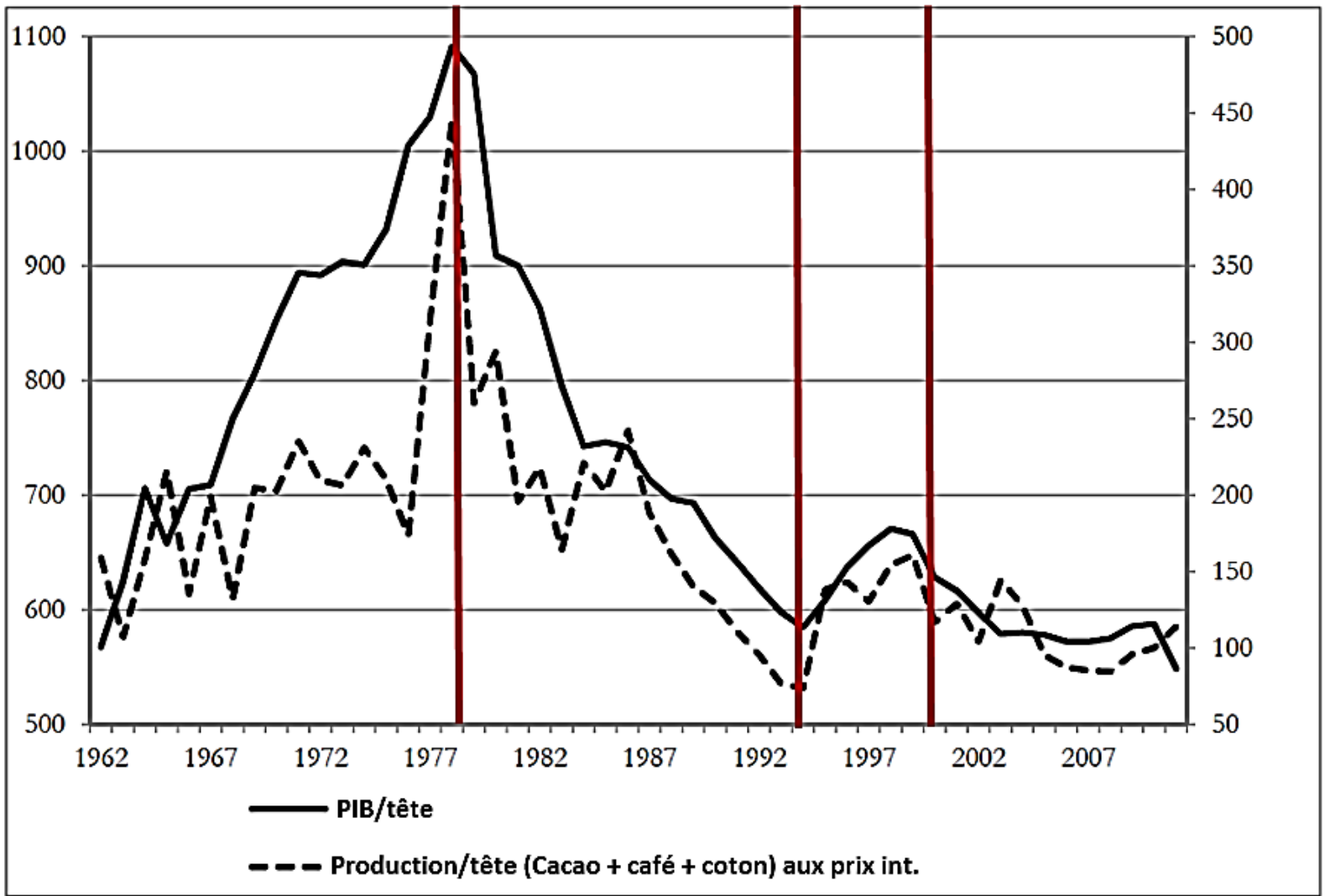

Note: En trait plein et sur l'échelle de gauche, le PIB par tête en dollars constant de 2000. En trait pointillé et sur l'échelle de droite, la production totale de cacao, café et coton, évaluée aux prix internationaux décalés d'un an, déflatés par le déflateur de PIB et convertis en dollars constant de 2000.

Source : Cognean et al. (2016)

3 On retrouve, à quelques spécificités et ajustements près, ces 4 grandes phases dans plusieurs travaux (Cogneau et al., 2016 ; Cogneau et Mesplé-Somps, 2002, Boussou, 2017 ; Kouassi, 2014) 
Au lendemain de son indépendance la Côte d'Ivoire va connaître une période faste de près de 20 ans avec une croissance économique soutenue, son PIB réel évoluant à un rythme moyen proche de $8 \%$ par an (Cogneau et Mesplé-Somps, 2002). Alors même que sur la période sa croissance démographique est aussi très forte (plus de 4\% par an), la Côte d'Ivoire parvient dans les années 1970 au statut de pays à revenu intermédiaire. Le succès est tel que la Côte d'Ivoire devient un exemple international, au point que le président américain Johnson l'évoque en ces termes : "To those that tell us that developing countries are really doomed (...) we have a simple answer. We say to them: Look at the Ivory Coast » (in Bamba, 2016, p. 117). Comme le soulignent Bouquet (2011) et Bamba (2016), les origines du « miracle ivoirien » remontent à la période coloniale et notamment la période de l'après seconde guerre mondiale. A cet égard, la stratégie de développement fondée sur l'exploitation de l'avantage comparatif agricole et forestier du pays s'inscrit dans cette continuité. Le développement extensif des agricultures d'exportation va alors bénéficier d'un environnement international favorable marqué en particulier par le «boom » des prix du café et du cacao. La production et les exportations agricoles (et de bois) vont croître à des rythmes très élevés (entre 7 et $10 \%$ par an) et représenter près de $80 \%$ des exportations sur la période (Berthélemy et Bourguignon, 1996). L'encadrement des prix aux producteurs à travers l'organisme de la Caistab (Caisse de stabilisation et de soutien des prix des produits agricoles - CSSPPA) a permis de leur assurer des revenus conséquents et relativement stables. Mais la Caistab a aussi permis à l'Etat de capter des parts considérables du surplus agricole. Ces surplus ont notamment servi à financer une politique volontariste de grands investissements dans les infrastructures, la santé et l'éducation et une politique salariale et de recrutement avantageuse dans la fonction publique (avec notamment dans les années 1970 le "décrochage » du salaire des enseignants, militaires et magistrats de la grille de rémunération officielle). Ainsi, tous les ivoiriens se sont sentis bénéficiaires du miracle. La stratégie consistait également à diversifier progressivement les structures productives à travers une politique d'industrialisation par substitution aux importations qui connaitra son apogée dans les années 1970 : en 1974 l'agriculture et l'exploitation forestière ne constituent plus que 30\% du PIB (contre 60\% dix ans auparavant (Berthélemy et Bourguignon, 1996).

Ce modèle de croissance extensif impulsé par Houphouët Boigny a ceci de particulier qu'il repose sur un savant équilibre entre ouverture libérale et planification keynésienne ${ }^{4}$. Son keynésianisme est tout incarné dans la régulation des marchés et des prix ainsi que dans un développement planifié au moyen de plans quinquennaux. Son libéralisme et son ouverture au capitalisme se traduisent par une forte extraversion. Une extraversion commerciale d'abord à travers les exportations agricoles, une extraversion des facteurs de production ensuite à travers sa dépendance aux capitaux étrangers (dette et aide publique au développement) et à une main d'œuvre étrangère (venue des voisins du Nord, Burkina Faso et Mali).

4 Au moment des indépendances, Houphouët Boigny fut l'un des rares parmi les nouveaux chefs d'État africains à ne pas choisir l'alignement sur le modèle soviétique mais l'ouverture au capitalisme. 
Dès la fin des années 1970 les signaux de la crise à venir apparaissent. Les germes de cette crise sont probablement intrinsèques au modèle de croissance de la période lui-même, croissance qualifiée de fragile par Samir Amin dès 1967 (Amin, 1967). Cette fragilité est tout autant exogène, liée à l'extraversion du modèle l'exposant aux retournements de conjoncture, qu'endogène, la logique extensive finissant toujours par atteindre ses limites (raréfaction des terres disponibles) et certains choix politiques s'avérant désastreux. Ainsi, la stratégie d'industrialisation par substitution aux importations bute sur un marché intérieur trop exigü et dérape dans une multiplication de créations industrielles, sociétés d'Etat et entreprises publiques, pour la plupart mal gérées, aux politiques d'investissement incohérentes et aux emprunts inconséquents (Bouquet, 2011; Cogneau et Mesplé-Somps, 2002). Le retournement de situation au niveau international, avec de manière concomitante la chute des cours des matières premières et la hausse des taux d'intérêt (suite à la réorientation de la politique monétaire américaine), conduit d'une part à une sévère dégradation des termes de l'échange pour le pays et, d'autre part, à un alourdissement de la charge de sa dette extérieure qui s'était creusée à la fin des années 1970. L’effet de ciseau est radical : le pays doit rembourser plus avec moins de recettes extérieures. Et, alors même que l'investissement privé réagit vite en se contractant, paradoxalement l'investissement public s'accroit, le gouvernement décidant d'augmenter ses dépenses d'investissement courantes qui atteignent 25,2\% du PIB en 1981 (Berthelemy et Bourguignon, 1996). La dette explose et de 1975 à 1980 le déficit public passe de 2,2\% à 11,8\% du PIB. Quant aux intérêts sur la dette, ils passent de 3,2\%, en 1980, à 8,3\% du PIB en 1983 (Bossou, 2017). Le pays entre alors dans une phase de succession de plans d'ajustements structurels (PAS) impliquant diverses mesures de restrictions budgétaires et de restructuration du secteur public. Le court rebond des cours internationaux en 1985-1986 a pu laisser penser à un début de stabilisation macroéconomique. Il n'en était rien, et le retournement des termes de l'échange fin 1986 marque le début d'une période de six ans (19871993) qui à maints égards peut être considérée comme le point culminant de la crise économique et financière et le point de départ des instabilités sociales et politiques. Après un dernier baroud d'honneur face aux institutions internationales et aux marchés internationaux (la fameuse "guerre du cacao » de 1987 à 1989), les autorités nationales finissent par céder et baissent pour la première fois depuis 25 ans le prix aux producteurs de café et cacao. L'échec est terrible. Alors qu'au milieu de l'année 1988 un planteur de cacao recevait 400 FCFA par kilo de fèves et que la Côte d'Ivoire représentait $40 \%$ du marché mondiale, fin 1989 ce même planteur ne reçoit plus que 150 FCFA par kilo et le pays ne représente plus que 20\% du marché mondial (Bouquet, 2011).

Dans le même temps, le pays s'engage dans la voie du multipartisme. Les élections de 1990 reconduisent à la tête du pays F. Houphouët Boigny, et surtout marquent la nomination d'un premier ministre, A. Ouattara. Surnommé le "FMI's boy », en raison de son passé au sein des institutions internationales, ce dernier à la charge de mettre en œuvre une sévère cure d'austérité, dans le cadre du quatrième PAS, caractérisée notamment par le gel des salaires des fonctionnaires, le raccrochage des salaires des enseignants, la compression des effectifs... L'incidence de la pauvreté bondit de 20 à 38\% (au seuil de 1.25\$) sur la période (1988-1993) (Cogneau et al., 2016). 
C'est le début des instabilités sociales et politiques avec des mouvements de mécontentement forts (jeunes, étudiants, enseignants descendent dans la rue, des mutineries apparaissent dans l'armée, des manifestations d'opposition en février 1992 sont violemment réprimées...).

\section{Une guerre de succession dans un contexte post-dévaluation (1993-1999)}

Félix Houphouët Boigny décède le 7 décembre 1993. C’est Henri Konan Bédié, président de l'Assemblée nationale et ancien de la Banque Mondiale qui lui succède. Lorsqu'il arrive à la tête du pays, la situation économique est calamiteuse (le PIB par habitant est retombé à son niveau de 1960). Nul n’ignore que l'ancien président était sans doute le dernier rempart à la mise en place de la dévaluation du franc CFA. Les conditions politiques étant réunies, celle-ci interviendra quelques jours après son décès, le 12 janvier 1994 (dévaluation de 50\% du F. CFA). L'ajustement monétaire vient ainsi s'ajouter aux mesures d'austérité budgétaires drastiques entamées depuis 1990 et aux réformes de libéralisation de l'économie qui s'accélèrent sur la même période (privatisations, nouveau code d'investissement, flexibilité accrue du marché du travail, abaissement du contrôle des prix avec en point d'orgue le démantèlement de la Caistab en 1999). Les effets de la dévaluation sont assez ambigus. A certains égards, c'est un succès. On assiste à une relance des exportations et une amélioration des équilibres extérieurs, le pays renoue avec la croissance économique (de l'ordre de 4\% de 1994 à 1998) et les comptes publics se redressent (le déficit global passe de 13,5\% du PIB en 1993 à 2,1\% en 1996) (Cogneau et Mesplè-Somps, 2002). Un nouveau plan de développement est défini pour la période 1995-2000. Baptisé «Les douze travaux de l'éléphant d'Afrique », il prévoit des programmes d'investissements ambitieux dans les domaines de l'énergie et du transport notamment financés et réalisés dans le cadre de nouvelles formes de partenariat public-privé sur le principe du «Build-Own-Operate Transfer» (BOOT). Pour autant, une partie de ce succès relève de facteurs conjoncturels favorables : tout d'abord, la remontée des cours du café et du cacao; ensuite, le nouveau dialogue instauré avec les institutions internationales favorise le retour de financements extérieurs publics (multilatéraux comme bilatéraux) et privés. Les réformes en cours ont donc très peu modifiés en profondeur le modèle de croissance (Cogneau et Mesplè-Somps, 2003). Plus encore, le retour de la croissance n'aura pas les effets de ruissellement escomptés sur la population qui auraient pu atténuer les problèmes sociaux engendrés par les PAS. Si l'accroissement de la pauvreté connaît un léger coup d'arrêt, les tensions sociales elles s'accélèrent avec la perspective des élections présidentielles de 2000. La loi sur le foncier rural de 1998 qui réforme complètement les droits coutumiers sur la terre fait reposer le principe de la propriété individuelle sur le droit du sang (les premiers occupants) et non sur droit du sol (ceux qui travaillent la terre). Elle instaure ainsi une inégalité de droits entre nationaux et étrangers qui attise les conflits fonciers et exacerbe les ressentiments identitaires. Dans le même temps, la corruption s'affiche à tous les niveaux (du quotidien des citoyens (Roubaud, 2003) au scandale du détournement des aides européennes, en passant par l'audit de la Caistab dévoilant une gestion très opaque de la filière café-cacao). En arrière-plan, la guerre des héritiers fait rage. Le 24 décembre 1999, le Général Robert Gueï s'empare du pouvoir à la faveur d'un coup d'Etat militaire. 
Le coup d'Etat de 1999 est le point de départ d'une longue période d'instabilités socioéconomiques et politiques en Côte d'Ivoire. Ces dix années de crise seront marquées par plusieurs évènements conflictuels illustrés dans la figure $\mathrm{n}^{\circ} 1$. Parmi ceux ci, on peut citer la période électorale de 2000, la seconde tentative de coup de d'Etat en 2002, les affrontements en 2004 entre les jeunes «patriotes » et les Français vivant en Côte d'Ivoire et la crise postélectorale de 2011. Aux élections de 2000, L. Gbagbo l'emporte face au Général R. Gueï (A. Ouattara ayant été une nouvelle fois exclu, et H.K. Bédié s'étant exilé en France). Son arrivée au pouvoir aurait pu laisser supposer que le modèle économique ivoirien allait être infléchi dans la mesure où le nouveau président se réclamait d'une idéologie plutôt opposée à l'économie de marché (son parti, le FPI, appartenait à l'Internationale socialiste). En fait, les turbulences politico-militaires qui marquèrent les dix années de sa présence à la tête de l'État l'empêchèrent probablement de peser véritablement sur l'économie. Il s'attacha néanmoins à mettre en place un mécanisme de régulation des prix du café et du cacao pour tenter de protéger les planteurs, mais le système s'avéra vite corrompu. Il en fut de même pour le contrôle de la production pétrolière, dont le pays attendait beaucoup puisque les réserves prouvées avoisinaient 225 millions de barils/jour, mais dont l'exploitation et les exportations demeurèrent opaques. Une des conséquences immédiates de ces instabilités sur le plan économique fut le ralentissement des investissements privés comme publics. Les grands projets d'investissement ont été suspendus et l'aide internationale s'arrêta. Les équilibres budgétaires furent mis à mal. Durant cette période, la croissance économique fut très largement interrompue avec plusieurs années marquées par des récessions. Le PIB par tête moyen retombera à un niveau proche de celui des années 1960, le Nord fut particulièrement touché. 
Figure $\mathrm{n}^{\circ} 1$ : Principaux évènements conflictuels et nombre de violences contre les civils entre 1997 et 2011

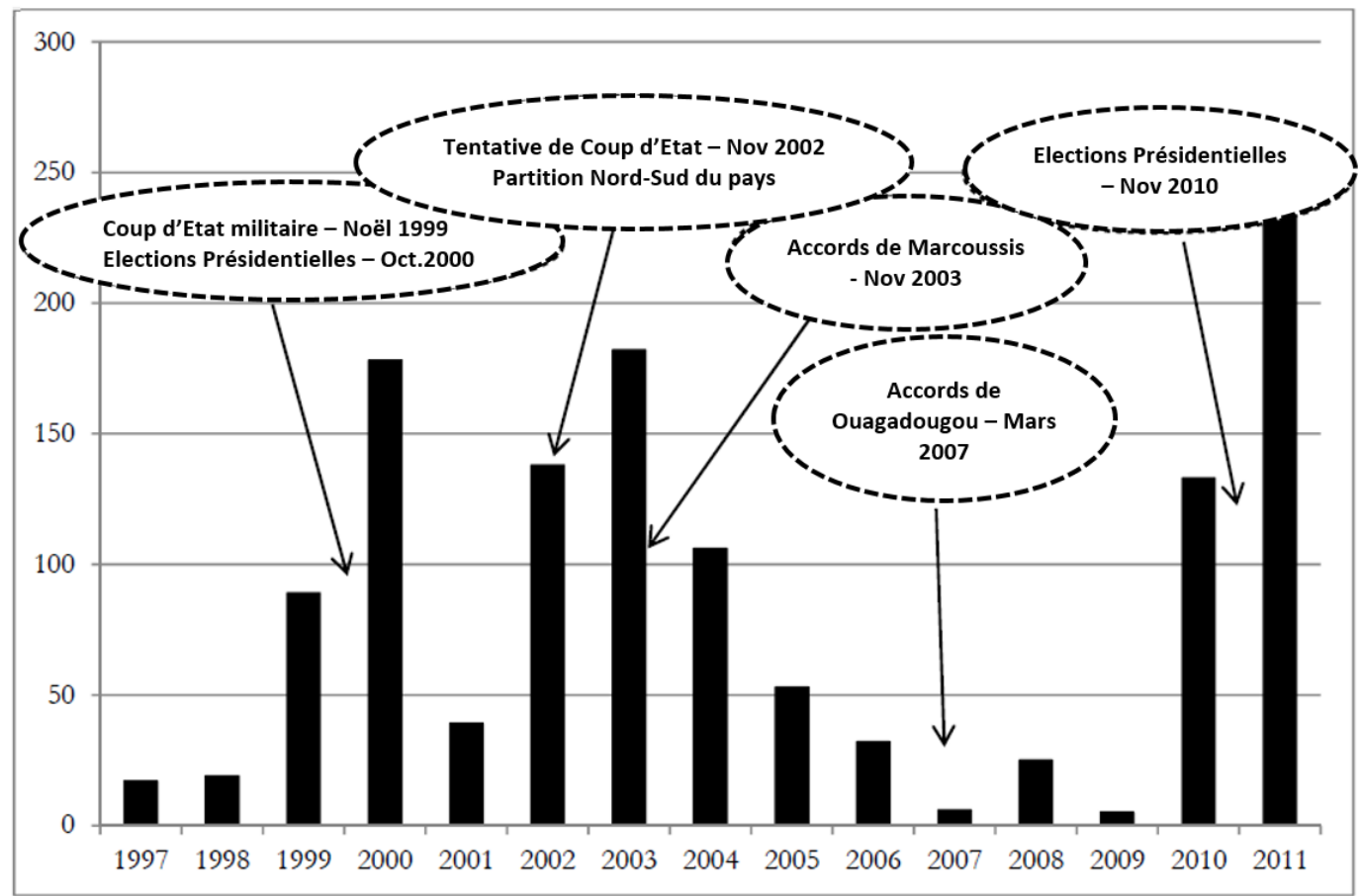

Note: Décompte des violences politiques contre les civils à partir des données de l'Armed Conflict Location and Event Data (ACLED). Les événements de violence politique signalés sont les violences contre les civils qui surviennent pendant les conflits civils, les combats, les émeutes ou les manifestations.

Source: Cognean et al. (2016)

Au moment où A. Ouattara prend ses fonctions en avril 2011, après dix ans de crise larvée et quatre mois de crise postélectorale suite aux élections de novembre 2010, l'économie du pays n'est pas totalement en ruine, puisque les principales matières premières d'exportation (café, cacao, pétrole) continuent d'être produites presque « naturellement» et que l'économie informelle a certainement assuré un rôle de "soupape »; mais la situation n'en est pas loin. Le taux de croissance cette année-là sera de $-4,2 \%$.

\subsubsection{Les fondamentaux du modèle de croissance ivoirien : transformations structurelles et enjeux de la période contemporaine}

Caractériser les fondements du régime de croissance ivoirien et ses transformations suppose d'articuler les facteurs premiers de la croissance, que sont les facteurs de production (terre, travail et capital) et leur productivité, avec les transformations structurelles qui les accompagnent (et/ou les soutiennent), tant au regard des structures productives que des modalités de l'insertion internationale et des compromis sociopolitiques. 
Bénéficiant d'une « rente forêt » importante (Cogneau et Mesplé-Somps, 2002, p.23), les richesses naturelles et agricoles ont toujours joué un rôle clé dans l'économie ivoirienne, de la période coloniale à aujourd'hui. A cet égard, le discours prononcé en 1987 par H. Boigny en introduction au plan directeur forestier 1988-2015 est éloquent : "La forêt a toujours été au centre des préoccupations de la Côte d'Ivoire depuis la création de notre pays. Tout est parti de la forêt. (...) Si une génération a disposé de la forêt ivoirienne pour assurer l'essor économique et social du pays, celle qui lui succède doit s'attacher désormais à la reconstituer. (...) Faire de la Côte d'Ivoire une nation forte dont le succès et la prospérité reposent et continuerons de reposer sur l'agriculture et son ambiance forestière » (cité par Bouquet, 2011, p.256). Le modèle de croissance ivoirien, qui peut être qualifié de modèle d'économie de plantation, repose donc sur l'exploitation extensive de cette rente forêt permise par une main d'œuvre abondante (croissance démographique et afflux de main d'œuvre étrangère). Il a finalement très peu changé depuis les indépendances. Certes, le poids de l'agriculture dans l'économie a diminué au cours des vingt-cinq dernières années, mais le secteur en lui-même s'est peu diversifié : "Le poids des cultures traditionnelles (patate douce, manioc, canne à sucre, huile de palme, plantain et cacao) est pratiquement resté inchangé entre 1990 et 2013, comptant pour approximativement 3/4 de la production totale 》 (Banque Mondiale, 2017, p.15). Si une stratégie de diversification agricole est en cours et que de nouvelles filières (re)voient le jour (caoutchouc, anacarde, coton, riz) (Ehrhart, 2015), la Côte d'Ivoire reste aujourd'hui fortement dépendante de l'exportation de quelques matières (le binôme cacao-pétrole a supplanté l'ancien binôme café-cacao) et vulnérable aux chocs sur ces produits (évolution des cours mondiaux, chocs climatiques et niveau des récoltes) ${ }^{5}$. Le ralentissement récent de la croissance et la grogne sociale chez les planteurs depuis la fin 2016 en sont une parfaite illustration. Le graphique $\mathrm{n}^{\circ} 3$ plus haut montre d'ailleurs très bien la forte corrélation entre l'évolution des revenus agricoles et celle du PIB par habitant. Les enjeux sont donc les mêmes, ils sont à la fois intra et intersectoriels. Sur le plan intra-sectoriel il s'agit de passer d'une logique d'exploitation extensive à une logique plus intensive et de montée en gamme par la transformation des produits. Sur le plan intersectoriel il s'agit d'encourager la diversification productive, notamment vers les secteurs industriels qui après une vague d'expansion jusque dans les années 1990 sont depuis restés stagnants (FMI, 2016). Ces enjeux sont particulièrement centraux afin de favoriser la création d'emploi de qualité à même d'absorber les flux de main d'œuvre à venir et de soutenir l'émergence d'une classe moyenne.

\section{Une main d'cuvre abondante mais un capital humain et des opportunités d'emplois formels limités}

Le modèle de croissance fondé sur l'exploitation extensive des ressources agricoles s'est effectué grâce à une main d'œuvre abondante. Cette dernière est le résultat d'une politique migratoire très ouverte et d'une croissance démographique naturelle dynamique (cf. supra). Si le taux de fécondité diminue depuis les années 1970, le taux de croissance de la population reste ainsi élevé

5 "En 2012, le cacao et le pétrole représentaient à eux deux $60 \%$ des exportations et $25 \%$ du PIB. En raison de la dépendance croissante du PIB vis-à-vis des exportations, qui représentent $53 \%$ du PIB en 2013 contre seulement $25 \%$ en 1965, selon la Banque centrale des États d'Afrique de l'Ouest (BCEAO), l'économie ivoirienne est de plus en plus exposée aux chocs sur ces deux produits 》 (OCDE, 2016, p. 52) 
et est même reparti à la hausse depuis 2005 (OCDE, 2016) (Graphique nº4). Cette croissance démographique s'accompagne d'une urbanisation croissante. La Côte d'Ivoire bénéficie aussi d'une population jeune importante et en forte croissance (en 2014, 78\% de la population a moins de 35 ans). Pour autant, le taux de dépendance n'est pas encore suffisamment réduit pour bénéficier du dividende démographique.

\section{Graphique $n^{\circ} 4$ - Le dynamisme démographique de la Côte d'Ivoire}
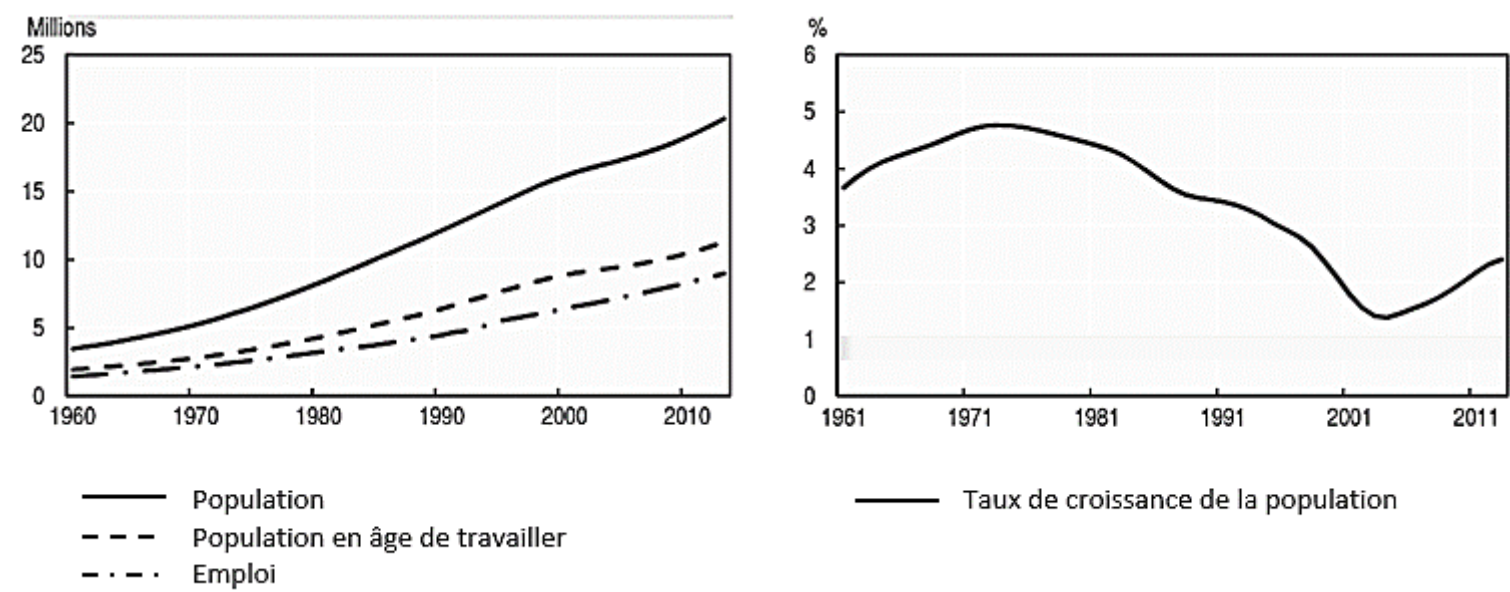

Source : OCDE (2016a, p. 41)

Les enjeux en termes d'emplois sont donc cruciaux et concernent à la fois l'accès à l'emploi via le capital humain (éducation et formation) et la création d'emploi. Sur ces deux points, des fragilités structurelles persistent. Concernant le premier, héritant à l'indépendance d'un système éducatif destiné à former des élites urbaines, la Côte d'Ivoire a entrepris des efforts importants en matière d'éducation, efforts visibles sur la période des "vingt glorieuses » (la fameuse « République des bons élèves »). Pour autant, au regard des efforts entrepris les résultats seront mitigés (Cogneau et Mesplé-Somps, 2002). Plus encore, les longues années d'instabilités et les dix années de crise politico-militaire ont ébranlé le système scolaire ivoirien et limité l'accroissement du stock de capital humain (on parle d’une génération sacrifiée) (Banque Mondiale, 2017 ; OCDE, 2016). Le niveau d'éducation reste faible et les systèmes de formation souvent inadaptés aux besoins des entreprises. Concernant le second, nous l'avons déjà souligné, le secteur informel reste le principal pourvoyeur d'emploi en Côte d'Ivoire (graphique $\mathrm{n}^{\circ}$ 5). Or les conditions de travail y sont souvent précaires et les niveaux de productivité très faibles. Cette prédominance de l'informel traduit par ailleurs la forte vulnérabilité des travailleurs dont très peu bénéficie de situation d'emploi stable et d'accès à la protection sociale. La création d'emplois de qualité est une condition sine qua non à la croissance des revenus des ménages et l'émergence d'une solide classe moyenne. 


\section{Graphique n ${ }^{\circ} 5$ - Situation de l'emploi en Côte d'Ivoire (2012-2015)}

Répartitition de l'emploi par branche d'activité

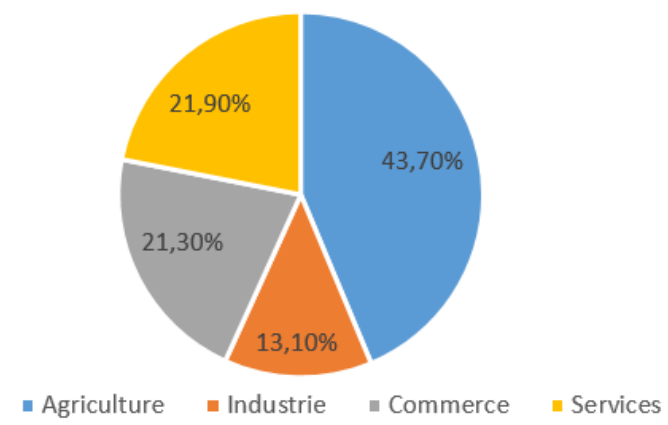

Source : ENV(2015) et ENSETE (2013)
Répartitition de l'emploi par secteur institutionnel

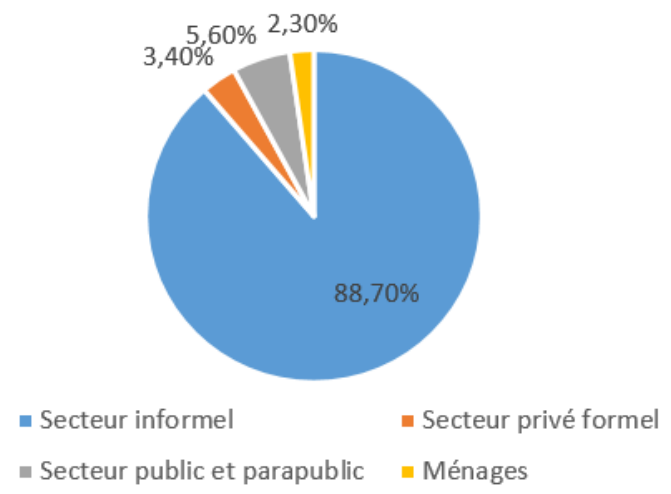

Un niveau d'investissement loin des standards des émergents : les enjeux du financement du capital

Ce dualisme du secteur productif, marqué par la prédominance de petites unités de production informelles (indépendants et micro et petites entreprises) au côté de quelques grandes entreprises n'est pas favorable à un investissement privé dynamique (les unités de production informelles investissant peu ou alors de manière extensive plus qu'intensive) ${ }^{6}$. Les stratégies des différents plans de développement ont ainsi souvent reposé sur d'importants investissements publics. Et, la prise de relais par les investissements privés, nationaux ou étrangers, s'est quant à elle généralement plus difficilement concrétisée. Aujourd'hui, l'essentiel du stock de capital ivoirien (immeubles, infrastructures de transport, télécommunications, énergie...) a en réalité été accumulé au moment des «vingt glorieuses »(OCDE, 2016). Sur ces vingt années, financée par les excédents de la rente cacao-café, l'accumulation du capital s'est faite à un rythme très élevé, notamment vers la fin des années 1970 (Cogneau et Mesplè-Somps, 2002). Une grande partie d'entre eux s'étant avérés être d'une rentabilité douteuse (mauvaise gestion), cet excès fut une des causes de la crise à venir. Et, déjà à l'époque, malgré l'ouverture aux capitaux étrangers, la dynamique des investissements privés n'avait pas été à la hauteur des attentes. A partir des années 1980, dans un contexte d'instabilités économiques, sociales et politiques, et de contraintes financières fortes les investissements publics comme privés commencent à diminuer. La dynamique d'accumulation est interrompue et le stock de capital va décroittre de manière quasi continue pendant 30 ans. L'ensemble des secteurs sont touchés, manufacturiers comme agricoles. Depuis 2011, dans un contexte de reconstruction post-conflit, on assiste à un redémarrage de la dynamique d'accumulation du capital portée une nouvelle fois par des plans d'investissement public (Banque Mondiale, 2015). Ces derniers bénéficient d'une situation financière assainie suite à différents allègements de dettes et à un soutien retrouvé auprès de la communauté internationale, y compris les investisseurs privés internationaux. Ces niveaux d'investissement, autour de $17 \%$ du PIB restent néanmoins insuffisants au regard des pays émergents dont les taux d'investissement se situent entre 25 et 35\% du PIB (OCDE, 2016). Pour pérenniser cette

\footnotetext{
${ }^{6}$ Voir Berrou (2014).
} 
dynamique, il faut que les investissements en cours privilégient les secteurs porteurs de diversification et d'accroissement de la productivité. Il faut aussi développer le financement endogène de l'investissement. Cela suppose notamment de renforcer les capacités d'épargne nationale en soutenant l'accroissement des revenus des ménages et en facilitant l'accès au secteur bancaire (réforme du secteur financier). On comprend alors aisément l'importance de l'émergence d'une classe moyenne étendue, tant la demande intérieure, moteur principal de la croissance, est aujourd'hui dépendante de flux financiers extérieurs (exportation, aide publique au développement, investissements directs étrangers).

Vers un modèle de croissance intensif? La productivité globale des facteurs

Les limites du modèle de croissance extensif de la Côte d'Ivoire ont déjà été soulignées. Le passage à une croissance plus intensive représente un enjeu de taille. Une manière d'évaluer ce degré d'intensivité de la croissance est de mesurer la contribution de la productivité globale des facteurs à cette croissance ${ }^{7}$. Si la période du premier miracle de la croissance ivoirienne avait été portée essentiellement par l'accumulation extensive des facteurs de production, le renouveau récent de la croissance à ceci de particulier qu'il repose à la fois sur des facteurs extensifs et intensifs (OCDE, 2016). L'accélération de la croissance sur la période récente ne semble donc pas pouvoir s'expliquer uniquement par un phénomène de rattrapage post-conflit (accumulation du capital). Il s'explique aussi, et presque à part égale, par une augmentation de la productivité globale des facteurs (Banque Mondiale, 2015a ; FMI, 2016). Cette dernière peut venir tout autant de progrès technologique que de transformations structurelles (réaffectation des ressources, en particulier le travail, des secteurs moins productifs vers les secteurs plus productifs). Il est encore trop tôt pour le dire mais, quoiqu'il en soit, pérenniser la dynamique de croissance en cours suppose de soutenir ces progrès de productivité (ce qui passe notamment par l'éducation et la formation, des infrastructures efficaces et des emplois de qualité). A cet égard, nul doute que la réduction des inégalités et l'émergence et la consolidation d'une large classe moyenne fait partie intégrante de ces enjeux (Berg, Ostry et Zettelmeyer, 2012).

\section{Des équilibres politico-économiques fragiles}

La dimension institutionnelle et les équilibres politico-économiques et sociopolitiques sont centraux pour comprendre un régime de croissance (Acemoglu et al., 2001, Rodrik, 2003). Il ne s'agira pas ici de rentrer dans une analyse approfondie de ces déterminants, dont une partie relève des mécanismes de redistribution et de stratifications sociales abordés dans la section suivante, mais juste de souligner quelques éléments saillants.

Le succès de la période des « vingt glorieuses » fut autant économique que politique. La stabilité du pays jusqu'au début des années 1980 semble en effet pouvoir être attribué au compromis politico économique mis en place par son premier Président Félix Houphouët-Boigny. Sorte de

\footnotetext{
Elle mesure l'efficacité combinée du travail et du capital et peut être considérée en ce sens comme une mesure du progrès technique et de l'efficacité de l'organisation du travail.
} 
"clientélisme éclairé» (Conte, 2004), ce "complexe politico économique » tel que le nomment Fauré et Médard (1982) reposait sur un équilibre subtilement entretenu entre élites du Nord et du Sud, Etat et milieux des affaires, bourgeoisie d'Etat et planteurs. "On était en plein dans la néopatrimonialisation de l'Etat, où la tête se servait largement, tout en redistribuant habilement autour d'elle pour mieux s'attacher les alliances» (Bouquet, 2011, p.254). Ces années fastes ont incontestablement permis de redistribuer la rente jusqu'aux niveaux les plus bas de la hiérarchie sociale tout en cherchant à réduire certains déséquilibres territoriaux. Ce compromis fragile reposait ainsi à la fois sur l'habilité politique du président dans le maniement du "dialogue à l'ivoirienne » (Fauré et Médard, 1982) et sur la disponibilité d'une large rente agricole principalement issue des filières cacao-café. A partir des années 1980, la crise économique et les ajustements structurels finissent par déliter le système clientéliste. Le point de rupture se situe très probablement dans la décision résignée de F. Houphouët Boigny de réduire drastiquement le prix garanti aux producteurs de cacao, décision mettant à mal la clé de voûte du système clientéliste fondé sur l'alliance historique du pouvoir avec les planteurs (Losch, 1997). Comme le souligne Dozon (2011), alors que le pays s'engage sur la voie du multipartisme, les compromis sociaux et politiques sur lesquels s'était façonnée la Côte d'Ivoire à l'époque de sa prospérité se fragilisent les uns les après les autres. Dans un contexte de crise économique et d'austérité sévères, la contestation sociale se généralise, chaque groupe voyant les fondements de son allégeance au «modèle ivoirien » se déliter.

Après le décès d'Houphouët Boigny, la guerre de succession qui s'engage entre les deux héritiers H.K. Bédié (Baoulé) et A. Ouattara (Dioula) consacre la fin de l'équilibre Houphouëtiste reposant sur l'alliance politique entre les élites du Nord et les élites Baoulé du Centre-Sud (Cogneau et al., 2016). La période qui s'ensuit marquée par un tarissement de la rente et "une libéralisation au pas de charge » (Cogneau et Mesplé-Somps, 2003, 90) n’a fait qu'accentuer les tensions et fragiliser les équilibres politiques. Après une période d'embellie économique suite à la dévaluation, le rétrécissement des marges de manœuvre à partir de 1996 conduit Bédié à réduire le périmètre de la redistribution clientéliste à un cercle de plus en plus restreint. Instrumentalisant le thème de l'«ivoirité » pour fédérer les populations du sud, notamment au moyen de la loi foncière de 1998, il ne fait que réveiller le sentiment de xénophobie et accroittre la division du pays (Conte, 2004). Après le coup d'Etat du Général Gueï, l'élection «calamiteuse » de L. Gbagbo en 2000 aurait pu ouvrir la voie à un nouveau compromis sociopolitique plus équilibré. Las, il reprendra largement le thème de l'« ivoirité » et entreprendra de former une alliance des autochtones du Sud (Cogneau et al., 2016). En 2002, le territoire est scindé en deux et le pays s'enfonce dans la crise politicomilitaire.

Il faudra attendre 2010 pour que soit organisée une nouvelle élection présidentielle, au cours de laquelle l'accord de 2005 entre les deux anciens frères ennemis du RDR (Ouattara) et du PDCI (Bédié), renouant avec le compromis Houphouëtiste pour créer l'alliance RHDP (Rassemblement des Houphouëtistes pour la Démocratie et la Paix), permet à A. Ouattara de remporter la victoire. Il est réélu en 2015 pour un second mandat. L'observation de la composition du gouvernement en 2016 témoigne assez manifestement d'un mode de recrutement des élites très fermé fruit des tractations clientélistes entre les deux partis. Cet équilibre est aujourd'hui très instable puisqu'au sein même du RDR des tensions se manifestent publiquement entre les élites pro-Alassane 
Ouattara et les pro-Guillaume Soro, actuel président de l'Assemblée nationale et ancien patron des Forces nouvelles (ex-rébellion). Ces derniers reprochent aux premiers de les avoir lentement mais sûrement mis sur la touche, "à tous les échelons de l'Etat, mais aussi dans le milieu des affaires »".

La fragilité et l'instabilité de ces équilibres politico-économiques dépendent pour beaucoup de la redistribution et de la répartition des richesses. L'analyse des processus de stratifications sociales en Côte d'Ivoire en donnera l'illustration et permettra de mieux comprendre la dynamique discontinue de formation des classes moyennes.

\subsection{Les dynamiques discontnues du processus de stratification sociale en Côte d'Ivoire : les classes moyennes, un espoir déçu plus qu'une classe constitué}

Le cas de la Côte d'Ivoire permet de déceler une historicité de longue durée du processus de différenciation sociale voire d'émergence de catégories " moyennes » depuis la colonisation, ce qui le rapproche des cas sud-africains et ghanéens en Afrique, voire de certaines situations asiatiques. Pour autant, les mutations erratiques du pays depuis les années 1960 n’ont cessé de briser ces processus de transformations sociales faisant de la classe moyenne ivoirienne plus un projet qu'une réalité, plus un espoir déçu qu'une classe constituée, plus un cumul de situations disparates qu'un ensemble cohérent disposant d'une capacité de mobilisation sociale et économique autonome. En s'appuyant sur les phases du régime de croissance identifiées précédemment, il s'agira d'analyser les fondements et les évolutions de ces processus de stratifications sociales en Côte d'Ivoire en portant une attention particulière aux catégories moyennes. Si la période post-indépendance s'inscrit dans une certaine continuité avec la période coloniale au regard l'émergence de catégories sociales intermédiaires (1.2.1.), les années 19802010, marquées par les instabilités économiques, sociales et politiques consacrent le déclin des classes moyennes ivoiriennes (1.2.2.). Le renouveau de la croissance économique sur la période contemporaine ouvre quant lui l'espoir de leur redéploiement sur fond d'inégalités criantes (1.2.3.).

\subsubsection{Les fondamentaux du modèle de croissance ivoirien: transformations structurelles et enjeux de la période contemporaine}

\section{L'émergence d'une petite bourgeoisie de planteurs ivoiriens durant la période coloniale}

Pendant la période coloniale, des ivoiriens éduqués ont été recrutés dans l'administration pour servir d'intermédiaires entre colonisateurs et colonisés, comme partout ailleurs en Afrique. Le choix colonial de faire de la Cote d'Ivoire un territoire dédié à la production agricole a ouvert la possibilité pour des planteurs, notamment dans le domaine du cacao, de s'investir dans ces activités et d'en retirer à la fois un revenu significatif et des capacités organisationnelles et

8 "Côte d'Ivoire : "Nous sommes ouverts à préparer l'alternance à M. Ouattara en 2020 », entretien avec Meïté Sindou ", Propos recueillis par Haby Niakaté, LE MONDE, consulté le 02.10.2017 à 17h46, http://www.lemonde.fr/afrique/article/2017/10/02/cote-d-ivoire-nous-sommes-ouverts-a-preparerl-alternance-a-m-ouattara-en-2020 $5195083 \quad 3212 . \mathrm{html}$ 
relationnelles notamment via le syndicat des planteurs de cacao et de café (Syndicat Agricole Africain 1944, Rassemblement Démocratique Africain 1946).

Ce début de processus de différenciation sociale est fondé à la fois sur des critères économiques et financiers et sur l'accès à des positions politico administratives. Il se constitue à la fois en rupture et en continuité avec les formes locales précoloniales de stratifications sociales et donne naissance à des élites africaines comme partout ailleurs. Emerge alors non pas une classe moyenne (l'expression étant souvent dévoyée pour dire intermédiaire entre le colon et les «indigènes ») mais bien ce qui a été qualifiée de "petite bourgeoisie de planteurs ivoiriens » (Chauveau et Dozon, 1988). Le développement de ces nouvelles activités économiques liées aux choix coloniaux génère de nouvelles situations et positions sociales (petits planteurs, fonctionnaires indigènes, politiques locaux au sein du RDA, syndicalistes locaux au sein du SAA...) qui modifient les modalités de la stratification sociale dans le pays. Toutes ces positions sociales constituent des catégories innovantes par rapport aux formes traditionnelles de segmentation, donnant accès à des niveaux de ressources monétaires inédits et à des ressources relationnelles et de pouvoir puisant dans l'ordre colonial imposé et dans leurs alliances ponctuelles (petite bourgeoisie de planteurs et petite bourgeoisie politico administrative) (Gastellu \& Affou, 1982 ; Fauré et Médard, 1982). Ces catégories sociales, ces «élites » sous la colonisation, sont « en suspension », leurs enjeux n'étant ni structurant, ni expression de ce que vit le reste de la population. Elles se construisent à la marge du système colonial et toujours en lien avec les appartenances communautaires, dans un mouvement d'accumulation progressif et différencié de ressources. Cette petite bourgeoisie de planteurs aisés, essentiellement rurale et fondée sur des intérêts fonciers va commencer à investir les emplois de l'administration et de la politique dans le monde urbain, notamment via les organisations syndicales et la promotion par l'école. De petits planteurs locaux à faible capacité profitent quant à eux des retombées de ce mouvement. Ils s'enrichissent mais sans disposer ni des capacités d'émergences ni des capacités d'action collective de cette petite bourgeoisie. Si Loucou (2012) identifie bien les dynamiques de transformation de la structure sociale à l'œuvre à cette époque, on ne peut en aucun cas le suivre dans son analyse identifiant l'émergence d'une structure de classe en trois groupes (ouvriers, classe moyenne, bourgeoisie), sauf peut-être à la réserver au monde de l'économie moderne.

Ainsi, à partir des années 1940 émergent en Côte d'Ivoire des groupes sociaux en voie de différenciation par leur capacité à investir à la fois le foncier et les activités agro industrielles, les activités bureaucratiques et politiques et à tirer parti d'un mouvement global d'expansion économique. Dans ces groupes, seul celui de la petite bourgeoisie de planteur peut être perçu comme disposant d'une identité collective active appuyée sur une capacité de mobilisation effective.

\section{Le miracle ivoirien et le développement d'une large «classe moyenne » aux contours hétéroclites}

Avec les indépendances cette petite bourgeoisie de planteur, encore très largement rurale, va continuer sa montée vers la ville et vers le statut d'élite dirigeante constitutive d'une véritable "bourgeoisie d'Etat», associant toujours davantage intérêts agricoles, économiques et politiques par 
des stratégies d'alliances familiales et de cumul de différentes positions stratégiques (Fauré et Médard, 1982). Elle a pu saisir alors l'ouverture des fonctions politiques et administratives (Chauveau, 2006), pour investir les postes de l'administration d'état (gouvernement, haute administration, entreprises publiques) et ainsi amplifier ses investissements dans l'agro-alimentaire et les diversifier dans d'autres secteurs. Cette bourgeoisie politico administrative ou ce que Gastellu et Affou (1982) qualifient de "bourgeoisie agraire», ne se construit en aucun cas comme une classe moyenne mais bien comme une «bourgeoisie d'Etat», un groupe dirigeant relativement fermé et aux intérêts toujours plus croisés. Ce groupe cumule les postes dominants de la politique, de l'administration d'Etat et du complexe agroindustriel et constitue la frange des 5 à $10 \%$ les plus riches du pays.

Mais d'autres transformations sociales sont à l'œuvre. Durant ces deux premières décennies postindépendance, le PIB réel par habitant croît à un rythme annuel moyen de près de 4\% (Cogneau et Mesplé-Somps, 2002). Au côté de la bourgeoisie d'Etat, le miracle économique ivoirien entraîne alors dans son sillage ce que Savina et al. (1986) ou Le Pape (1997) qualifient, avec plus ou moins de précautions, de «classes moyennes ». Bossuroy et Cogneau (2013) montrent que les deux premières décennies suivant l'indépendance ont permis une forte croissance des emplois salariés non-agricoles bien rémunérés. Les mobilités du secteur rural vers les emplois salariés urbains ont alors fortement augmenté, beaucoup d'enfants d'agriculteurs basculant vers ces nouveaux secteurs. Cette expansion du nombre d'emplois urbains, tout en profitant à la bourgeoisie d'Etat, a aussi profité à tout un ensemble de petits planteurs et de jeunes éduqués accédant à des postes intermédiaires de la fonction publique et à des emplois qualifiés et de techniciens des entreprises privées. Ces «classes moyennes» bénéficient par ailleurs d'un appui direct de l'Etat qui en favorise le développement en les soutenant via un système de redistribution clientéliste systématique de la rente (Conte, 2004). Cela se traduit notamment par une politique salariale et de recrutement généreuse dans la fonction publique (avec notamment le recrutement automatique des diplômés, puis le « décrochage» de certaines catégories), des prêts bonifiés pour leur faciliter l'accès à la propriété immobilière urbaine (et leur permettant également de diversifier leur revenus), et des politiques d'infrastructures visant à l'amélioration générale des conditions de vie (Cohen 1974 ; Savina et al., 1986 ; Marie 1988 ; Dubresson, 1989). L’Etat a aussi soutenu l'émergence d'une classe moyenne de planteurs en incitant à l'investissement dans les plantations via notamment une politique d'encadrement des prix avantageuse. En milieu rural, les cultivateurs de café et de cacao ont ainsi pu constituer à cette époque une catégorie sociale assimilable à une classe moyenne, sans compter les "planteurs absentéistes》 de la fonction publique ou du secteur privé cherchant à diversifier leurs revenus (Affou Yapi, 1987). Se construit ainsi un début de différenciation sociale dépassant l'opposition classique minorité de riches et océan de pauvreté au profit de l'émergence d'une masse intermédiaire issue d'une pauvreté en forte réduction et fondée sur la diversification des activités économiques et une politique de soutien explicite de la part de l'état. Dans le même temps se dessinent aussi des rapports de classe fondés sur une différenciation de genre : tandis que les emplois salariés et de petits entrepreneurs du secteur moderne sont monopolisés par les hommes, les femmes (et les étrangers) sont confinées dans les emplois de l'informel (petit commerce, artisanat, multiactivité) (Vidal, 1991). Durant cette période «heureuse » du miracle ivoirien les bases de classes moyennes en devenir sont donc posées 
comme l'illustre à merveille la Bande Dessinée Aya de Yopougon. Savina et al. (1986), estiment qu'en 1978 à Abidjan ces « classes moyennes » représentent environ 345000 personnes, soit 28\% de la population de la métropole, et sont composées de cadres, d'enseignants, diplômés et qualifiés du secteur tertiaire, de travailleurs qualifiés, d'entrepreneurs et commerçants du secteur moderne. Cette classe moyenne apparait très brouillée, hétéroclite et hétérogène comme l'analyse avec finesse Marie (1988) qui en souligne les nouvelles pratiques (consommation, épargne, éducation, habitat...) qui affectent la structuration sociale d'ensemble, la physionomie de l'espace urbain et les trajectoires sociales futures.

Durant ces années fastes de nouveaux rapports sociaux émergent et modifient le mode de production ivoirien. D’une part, la catégorie de la bourgeoisie d'Etat associée aux grands intérêts économiques étrangers localisés, ne cesse de se différencier du reste de la population avec laquelle elle n'entretient plus que des rapports clientélistes fortement rattachés à des appartenances villageoises historiques (soutien politique contre accès privilégiés aux emplois et petits marchés publics) particulièrement manifestes lors des funérailles ou des visites de personnalités politiques (Durand Lasserve et al., 1985). D’autres part, des catégories intermédiaires massives, en voie d'enrichissement, au mode de vie très occidentalisé (Touré, 1981) et qui aspirent à suivre les riches, dont ils sont proches par les origines mais dont l'enrichissement des premiers ne cesse de les éloigner, tendent à former un «monde du milieu » très hétéroclite et sans unité sociale bien plus qu'une classe moyenne. La période du miracle ivoirien, avec un système de redistribution de la rente savamment orchestré, a pu laisser penser à une continuité de situation entre ces « nouveaux » gens du milieu et les riches, à une « compatibilité d’intérêt » (Le Pape, 1997), mais ce n'était probablement qu'un leurre. Deux arguments au moins peuvent être soulignés ici. Tout d'abord, comme le montrent Durand Lasserve et al. (1985), si les consommations de tous les jours devenaient relativement proches en revanche le contrôle du capital économique et du foncier agricole ne cessait de souligner le creusement des écarts, de même que la nature et la qualité des habitats urbains (ce qu'on peut clairement voire aussi dans Aya de Yopougon). Ensuite, les travaux de Cogneau et al. (2006), Cogneau (2007) et Bossuroy et Cogneau (2013) montrent qu'une fois isolés les effets de mobilités structurelles liées aux transformations de l'emploi accompagnant la croissance économique (création d'emplois urbains), la mobilité sociale nette en Côte d'Ivoire sur cette période est en réalité extrêmement faible, bien inférieure à celle que l'on peut observer à la même époque au Ghana ou en Ouganda (deux anciennes colonies britanniques). Ces auteurs montrent que cette mobilité sociale nette en Côte d'Ivoire s'est réduite entre les générations d'avant et d'après les indépendances. Dans les générations d'avant l'indépendance, pour un individu dont le père était agriculteur et pour un individu dont le père ne l'était pas, la probabilité de reproduire les positions paternelles est près de six fois supérieure à la probabilité de les échanger. Ce coefficient de reproduction passe à douze après l'indépendance.

Ce décrochage entre riches et catégories intermédiaires deviendra finalement flagrant avec la différenciation des situations économiques des deux catégories face à la crise, entraînant des conséquences en termes de mobilisation politique non négligeables. 


\subsubsection{Le long déclin des classes moyennes ivoiriennes et la fin du mythe de l'assimilation aux riches}

La crise, les ajustements structurels et le phénomène des «conjoncturés 》 (1980-1993)

«L'ébranlement » des années 1980 va mettre un coup d'arrêt à ce processus d'émergence des classes moyennes et "conjoncturer »" les aspirants à l'émergence sociale tout comme une partie des classes moyennes stabilisés (Dubresson, 1989 ; Le Pape, 1997). La longue période de crise économique et financière sévère que va connaitre le pays et la succession de programmes de stabilisation et de réformes structurelles (restrictions budgétaires, restructuration du secteur public, libéralisation du secteur privé) vont impacter durablement les conditions de vie des populations. La chute du niveau de vie de l'ensemble de la population ivoirienne est drastique: la dépense moyenne nationale par tête s'est réduite presque de moitié entre 1985 et 1993 (Cogneau et Mesplé-Somps, 2003)

Une des manifestations de cette crise est la dégradation de la situation sur le marché du travail. Avec la compression des effectifs de la fonction publique, la réduction des salaires et la destruction de l'emploi salarié dans le secteur privé formel, le marché du travail devient de plus en plus segmenté et on assiste à une prolifération des activités informelles. Cette explosion du secteur informel exprime très bien la crise que traversent ces catégories sociales « moyennes » sevrées du soutien de l'état et directement affectées par sa restructuration. La protection d'une partie stratégique de ces gens du milieu (enseignants, militaires, magistrats) par leur décrochage de la fonction publique décidée en 1976 par Félix Houphouet-Boigny cessera d'ailleurs en 1991. Les classes moyennes, directement ou par l'intermédiaire de leurs héritiers ainsi que les candidats naturels à leur ralliement (les diplômés) sont frontalement « conjoncturées » dans tous les secteurs de leurs investissements et espaces de revenus. Pour une bonne partie, ces gens du milieu, effectifs ou aspirants, sont contraints de se lancer dans des "micro et petites activités urbaines de production et de commerce 》 (Fauré et Labazée, 2000). Ces activités informelles, fonctionnant essentiellement sur fonds propres, s'inscrivent dans une logique d'accumulation extensive fondée sur la pluriactivité plutôt que sur l'accumulation intensive du capital et la croissance interne (Fauré et Labazée, 2000; Socko, 2011). Ce délitement du mode de production ivoirien s'accompagne ainsi d'une fragilisation des catégories intermédiaires dont certaines retombent dans la pauvreté tandis que sont bloqués les nouveaux flux d'émergence sociale.

Il en résulte une expansion considérable de la pauvreté et des inégalités, expansion particulièrement marquée sur la fin de la période (1988-1993) où les effets de la crise économique et financière s'étendent à tout le pays et à toutes les catégories sociales (y compris les planteurs suite à la diminution des prix garanties) (Graphique n⿳5 et tableau nº ${ }^{\circ}$ ). Entre 1985 et 1993 l'incidence de la pauvreté au seuil de 1,25\$ triple passant de $10 \%$ à plus de $30 \%$. La transmission intergénérationnelle des statuts sociaux est entravée, pour la première fois le taux de scolarisation

9 Expression de la rue reprise notamment par Contamin (1997). Les Ivoiriens avaient même surnommé «Conjoncture » la nouvelle bouteille de bière que la brasserie locale vendait sans augmentation de prix mais avec un tiers de liquide en moins (Bouquet, 2011). 
primaire diminue et on constate un impact sérieux sur la nutrition des enfants (en particulier chez les agriculteurs) (Cogneau et Mesplé-Somps, 2002, 2003). Si la région d'Abidjan reste encore protégée (5\% de la population sous le seuil de pauvreté en 1993), la pauvreté urbaine s'accroit considérablement dans les villes secondaires (de $8 \%$ à plus de $30 \%$ ).

Graphique n 6 - Evolution de l’incidence de la pauvreté entre 1985 et 2015 (\%)

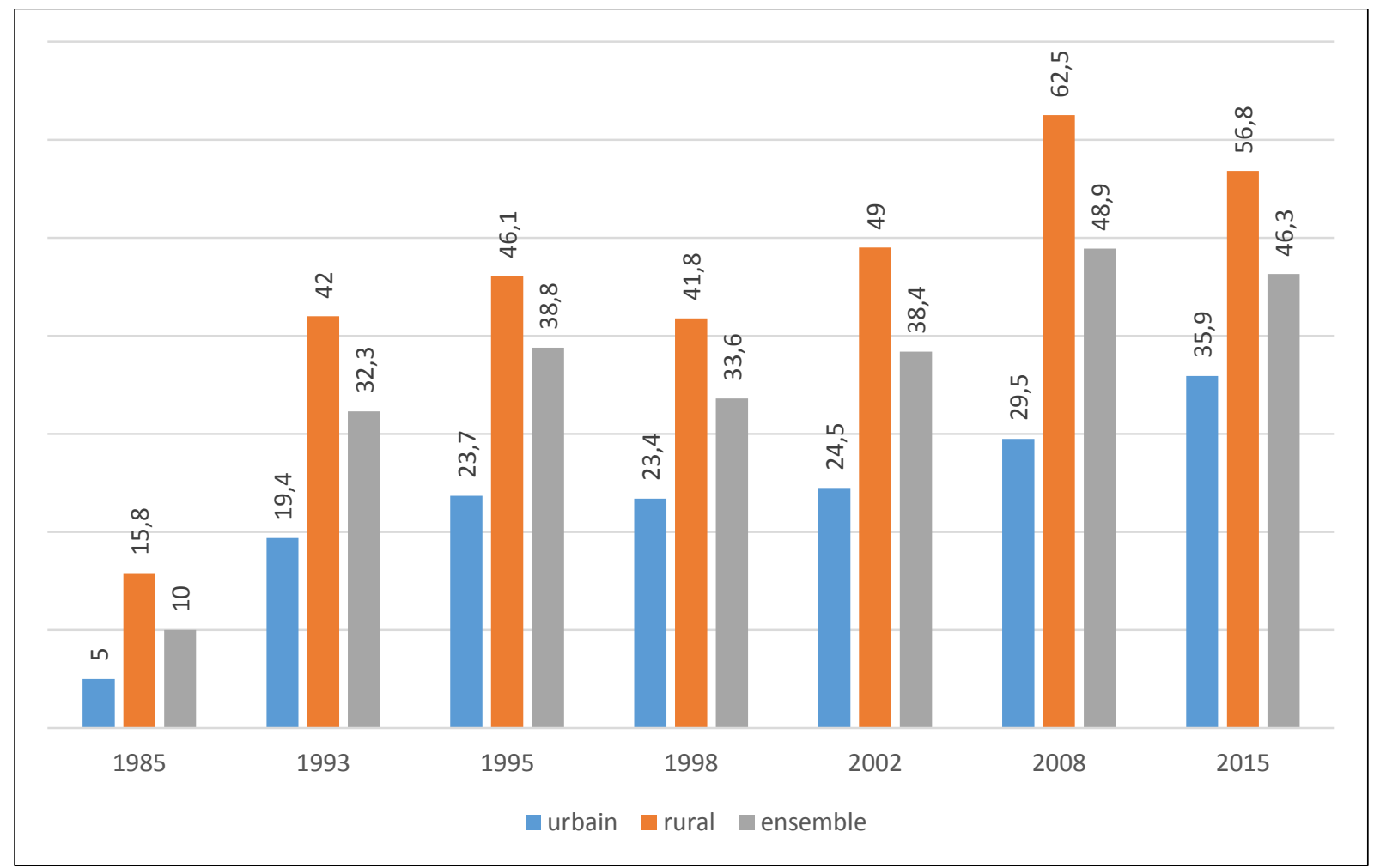

Source : ENV (2015)

Les inégalités de revenus s'accroissent également fortement. Sur la période 1985-1988 l'indice de GINI de la Côte d'Ivoire est estimé à 0,56 en comparaison de celui du Ghana qui estimé à 0,46 (Cogneau et al., 2006 ; Cogneau, 2007). Aux inégalités intersectorielles consécutives au dualisme rural (agricole) / urbain (non agricole) particulièrement prononcé dans les pays francophones, s'ajoute l'accroissement des inégalités intrasectorielles. En milieu urbain d'abord, avec comme on vient de la voir la segmentation croissante du marché du travail et l'urbanisation de la pauvreté (le coefficient de GINI du revenu des non agriculteurs est estimé à 0,59). Mais en milieu rural également avec des écarts croissants entre agriculture de rente et petite paysannerie dans un contexte de baisse des prix agricole et de libéralisation de la filière. Ces inégalités vont s'accroître de manière encore plus criante dans le contexte post-dévaluation, consacrant de manière presque irrémédiable la rupture en cours entre les riches et les classes moyennes émergentes. 
Tableau n ${ }^{\circ} 1$ - Evolution de l'incidence de la pauvreté selon le statut socioéconomique du chef de ménage $(\%)$

\begin{tabular}{|l|l|l|l|l|l|}
\hline Statut du chef de ménage & $\mathbf{1 9 8 8}$ & \multicolumn{1}{|c|}{$\mathbf{1 9 9 3}$} & $\mathbf{1 9 9 8}$ & $\mathbf{2 0 0 2}$ & $\mathbf{2 0 0 8}$ \\
\hline Agriculture & 30 & 52 & 48 & 51 & 59 \\
\hline Indépendant (auto-emploi) & 8 & 26 & 22 & 26 & 32 \\
\hline Secteur public & 6 & 10 & 7 & 10 & 7 \\
\hline Secteur privé formel & 2 & 17 & 14 & 18 & 21 \\
\hline Sans emploi & 18 & 35 & 22 & 22 & 31 \\
\hline
\end{tabular}

Note: Le senil de pauvreté utilisé par Cognean et al. (2016) pour la période 1988-2008 est celui de 1,25\$ PP A par jour

Source : Cogneau et al. (2016)

Les effets de la dévaluation et la « séparation irréversible » entre l'élite dominante et les autres (1994-1999)

Comme le remarque Le Pape (1997), après vingt années de miracle économique ivoirien qui avait laissé penser aux classes moyennes qu'elles étaient assimilables aux dominants, l'évidence d'une rupture de destin et d'identité s'est imposée. "La stratégie d'ajustement suivie depuis 1994 a impliqué un changement des règles du jeu qui prévalaient depuis vingt ans en matière de répartition du produit. La "république des bons élèves" semble avoir puni ses enfants, c'est-à-dire les fonctionnaires et les salariés instruits du secteur moderne. »(Cogneau et Mesplé-Somps, 2002, p. 86).

Comme nous l'avons vu dans la première section, la situation économique post dévaluation a pu à certains égards être considérée comme un succès, notamment au regard des critères des institutions internationales (croissance, équilibres internes et externes). Pour autant, si l'extension de la pauvreté sous ses différentes formes, monétaires et non monétaires (revenu, conditions d'existence, nutrition, scolarisation), a connu un léger coup d'arrêt, cela ne s'est pas traduit par un retournement significatif de tendance (Cogneau et Mesplé-Somps, 2003). Plus encore, les réformes libérales engagées n'ont en rien modifié les fondamentales sociopolitiques du modèle de croissance. Les évaluations empiriques des effets de la dévaluation le montrent de manière éloquente, la croissance retrouvée entre 1995 et 1998 s'est surtout traduite par une amélioration du niveau de vie des ménages les plus riches, en milieu urbain comme rural (Grimm et al., 2002 ; Grimm, 2004, Cogneau et al., 2016). Si le coefficient de GINI au niveau national passe de 0,49 à 
0,51 entre 1993 et 1998, les inégalités augmentent fortement à Abidjan (de 0,49 à 0,53) comme en milieu rural (de 0,42 à 0,49) (Cogneau et Mesplé-Somps, 2003).

D’un côté, les riches réussirent à sauver leurs intérêts en se lançant dans ce que Conte (2004) décrit comme un processus de "partage des dépouilles du clientélisme houphouétiste ». Cette élite dominante qui détient le foncier rural ou du capital dans les grandes entreprises, notamment étrangères et exportatrices, va à la faveur de la dévaluation bénéficier d'une forte appréciation de ses avoirs extérieurs et profiter pleinement des processus de libéralisation et privatisation en cours pour étendre ses investissements dans les entreprises nouvellement privatisées. La bourgeoisie politico administrative continue donc à recycler ses intérêts grâce à sa capacité à cumuler positions dominantes dans les secteurs politico-administratifs et économiques et à en jouer pour construire des alliances autour des privatisations avec des intérêts économiques étrangers notamment (Conte et Sindzingre, 2002 ; Contamin et Memel Foté, 1997). Cela vaut pour l'économie de plantation où se sont les «gros planteurs» qui ont bénéficié du rebond de croissance et des prix des cultures de rentes. Plus largement, cette élite profite des pratiques de corruption ou de redistribution clientéliste de la rente. La rente cacaoyère d'une part, pour laquelle l'audit de la Caistab, démantelée en 1999, soulèvera le voile sur ces pratiques. La Banque Mondiale le rapporte en ces termes : "L'Etat qui prélève entre le quart et le tiers du prix de vente du cacao à sa sortie de Côte d'Ivoire, continue de capter l'essentiel de la manne cacaoyère, ainsi qu'une élite paysanne qui se remplit les poches à travers une multitude d'institutions inutiles et cô̂teuses au rôle non justifié, qui abusent de l'utilisation du prélèvement parafiscal » (cité par Bouquet, 2011, p.267). La rente de l'aide publique au développement d'autre part, avec le scandale du détournement des aides européennes. D'un autre côté, les autres catégories sociales, et notamment les classes moyennes largement dépendantes de la structure de l'Etat néopatrimonial houphouetiste (Médard, 1991) ne disposent pas des mêmes opportunités et voient leur situation se dégrader. Le nombre des retours vers la pauvreté s'accroit tandis que les processus de transmission intergénérationnelle de la position sociale moyenne se grippent.

Ce contexte d'accroissement des inégalités et d'appauvrissement va alimenter les tensions sociales sur fond d'instrumentalisation identitaire à travers le concept d'ivoirité. Les disparités spatiales entre villes et campagne et entre nord et sud en matière d'éducation, de santé, mais aussi de transport, de communications, d'électricité et d'eau, vont devenir criantes. L'accroissement de la pauvreté dans les zones rurales (au Nord notamment) et dans l'informel urbain va accroitre les tensions en natifs ivoiriens et immigrés d'origine africaine. La raréfaction des terres et l'accroissement des inégalités de revenus en milieu rural vont participer de la montée des conflits autour de l'accès au foncier entre autochtones et allochtones. Conflits que la loi de 1998 ne fera qu'exacerber. "En conséquence, les frustrations n'ont cessé de croître : celles de la petite bourgeoisie citadine souffrant d'une "séparation irréversible" (Vidal 1997) entre elle et l'élite dominante; celles de jeunes générations citadines éduquées, sans espoir d'embauche dans le secteur formel ; enfin, celles des petits paysans, insuffisamment bénéficiaires de la croissance, souffrant de carences importantes en matière de services publics et de plus en plus mobilisés par des conflits d'accès aux ressources. » (Cogneau et Mesplé-Somps, 2003, p.103) 
Cette rupture dans la formation d'une classe moyenne dans les années 1990 ne peut être sousestimée dans la compréhension des contestations sociales et les conflits de la fin des années 1990 et des années 2000 .

\section{La paupérisation globale de la société ivoirienne dans un contexte de crises militaro politiques}

La succession et l'accumulation de crises économiques, politiques, et militaires des années 2000 a paralysé une économie atone et conduit à une paupérisation globale de la société ivoirienne. Entre 2002 et 2008, le PIB par tête tombe en dessous des $600 \$$ (\$ constant de 2000), autrement dit revient à un niveau proche du PIB par tête du début des années 1960 (Cogneau et al., 2016). L'incidence de la pauvreté passe dans le même temps de 38,4\% à 48,9\% (Graphique nº). Dans ce processus, le Nord du pays fut particulièrement touché.

Les groupes qui étaient assimilables aux classes moyennes sont en voie de disparition. Les crises à répétition ont bloqué la poursuite du processus de différenciation sociale, affecté la reproduction sociale au sein des classes moyennes en fragilisant, voire en empêchant, le transfert intergénérationnel de capitaux financiers, sociaux et culturels. Une forme de redistribution vers le bas des catégories sociales de la «classe moyenne » s'est mise en œuvre. Elle est marquée par une paupérisation des fonctionnaires et des salariés du privé (Bouquet, 2013), une baisse significative du nombre de salariés des secteurs privé et public et en contrepartie une explosion des initiatives dans le secteur informel avec en 2002 dans l'agglomération d'Abidjan 610000 unités de production informelles (UPI), employant 950000 personnes dans les branches marchandes non agricoles (INS, 2005). Fauré et Labazée (2000) analysent en particulier la situation des petits patrons, promoteurs et entrepreneurs qui sont très proches de ces catégories en marge de la bourgeoisie politico administrative et d'affaires mais en partie connectée à elle dans le secteur commercial.

\subsubsection{Le retour des classes moyennes ivoiriennes? (2010-)}

Depuis 2011, la reprise très rapide de l'économie, dopée par des plans d'investissements ambitieux et un soutien retrouvé de la communauté internationale et des investisseurs internationaux, contribue à booster le pouvoir d'achat et la consommation des ménages. Impulsée également par des mesures politiques volontaristes, la croissance de la consommation des ménages peut préfigurer ou laisser penser à la reconstitution d'une classe moyenne. Le PIB par habitant croît sur la période à un rythme proche des $6 \%$ par an (WDI, 2017). Le gouvernement a commencé en 2014 à lever le gel des salaires pour les fonctionnaires de l'administration publique qui avaient exprimé leur mécontentement lors de plusieurs mouvements de grève en 2012 et en 2013 (FMI, 2013). Il a également soutenu un accroissement significatif du SMIG passant de 36607FCFA à 60000CFA, soit un quasi doublement. La réforme du secteur café-cacao s'inscrit aussi dans cet objectif de soutenir au mieux le revenu des ménages agricoles (même si la période actuelle en montre toute les limites). Des programmes d'accès au logement d'envergure sont lancés. Du côté des investisseurs privés, internationaux notamment, la 
réémergence de ces classes moyennes ne fait alors presqu'aucun doute, en particulier pour des marchés concentrés sur quelques villes et notamment Abidjan. En témoigne, par exemple, la construction d'un mall flambant neuf conçu par CFAO, l'ex-Compagnie française de l'Afrique occidentale, en partenariat avec Carrefour. Le PlaYce Marcory a été inauguré le 18 décembre 2015, avec la présence du Président de la République Alassane Ouattara, et ne compte pas moins de 46 boutiques commerciales venue répondre aux besoins anticipés d'une classe moyenne supposée en plein essor. Carrefour vient même d'inaugurer en juin 2017 son second centre commercial dans le quartier de Cocody-Riviera Palmeraie.

Pour autant, le recul analytique manque pour délimiter précisément l'ampleur du phénomène et décrire en profondeur les dynamiques de stratifications sociales à l'œuvre. Il est bon de rappeler que ces dynamiques s'inscrivent dans un contexte de croissance économique forte mais peu inclusive (cf. 1.1.). A cet effet, les travaux récents de Cogneau et Szajka (2016) et Sazjka (2017) montrent que le niveau des inégalités de revenus en Côte d'Ivoire sur la période récente est particulièrement élevé. Les auteurs, dans une démarche méthodologique et technique originale, combinent les données habituelles issues des enquêtes ménages (ENV, 2015) et les données issues des administrations fiscales collectant l'impôt sur le salaire (pour l'année 2014) ${ }^{10}$. Les premiers résultats de ces travaux tendent à confirmer que les enquêtes auprès des ménages et les évaluations des inégalités par les dépenses de consommation plutôt que les revenus conduisent à une sous-estimation du niveau des inégalités en Côte d'Ivoire comme dans nombre de pays d'Afrique subsaharienne. Les données ENV (2015) montrent que les 10\% les plus riches de la population représentent 32,5\% du revenu national, et les $1 \%$ les plus riches, $8 \%$. Les données fiscales sur les salariés du secteur public et privé formel montrent que ce groupe de la population est extrêmement inégalitaire (en particulier dans le privé). Dans ce groupe, les 10\% les plus riches représentent près de $40 \%$ du revenu total du groupe, les $1 \%$ les plus riches, $15 \%$. Ces derniers chiffres témoignent d'un niveau d'inégalité pas si éloigné de niveaux de pays très inégalitaires comme les Etats Unis où sur la dernière décennie, les $1 \%$ les plus riches captent $17,5 \%$ du revenu total. S'ils sont encore à prendre avec précautions, ces travaux pointent le doigt sur les risques d'un sérieux problème de répartition des fruits de la croissance. Or, dans le même temps, les données de l'ENV 2015 conduisent à une estimation de la pauvreté toujours aussi élevée avec près de la moitié de la population sous le seuil de pauvreté. Une pauvreté qui frappe particulièrement les agriculteurs, puis les travailleurs des secteurs formels et informels dont les niveaux de pauvreté sont assez proches.

\footnotetext{
${ }^{10}$ Ces données fiscales concernent donc les 361172 salariés (180669 dans le secteur public, 180503 dans le privé) qui payent leurs impôts et taxes à la CNPS. Ces derniers ne représentent évidemment seulement $4.2 \%$ de la population active et $5.6 \%$ de la population en emploi.
} 


\section{Graphique $\mathrm{n}^{\circ} 7$ - Incidence de la pauvreté selon statut socio-économique du chef de ménage en $2015(\%)$}

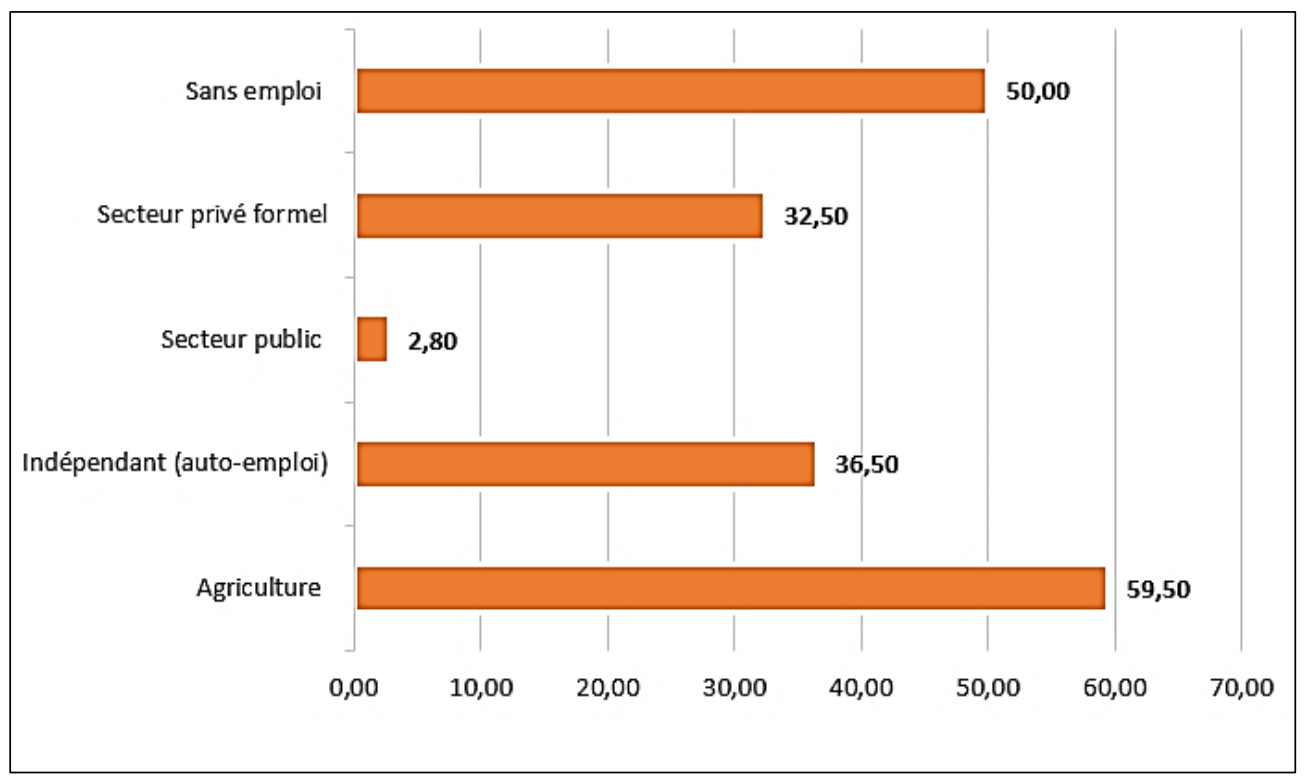

Source : ENV (2015)

Ainsi, sur le plan des logiques de stratification sociale à l'œuvre, il semble probable que la bourgeoisie d'Etat, protégée contre les effets de la crise par le haut niveau de capitaux de toutes natures accumulés, retrouve le chemin de l'enrichissement sur les mêmes bases que précédemment. Pour cette dernière, le cumul des positions dominantes dans les différents espaces d'activité (économique, politique et bureaucratique) demeure essentiel. Cela se traduit par exemple, comme le montrent Tarouth et Colin (2016) et Colin et Tarouth (2017) à propos de l'hévéaculture, par des investissements importants dans l'agro-industrie. Ces investissements sont fortement enchâssés dans des réseaux et logiques sociopolitiques pouvant s'accompagner de formes de corruption élevées ou de collusions de forme corporatistes. Ces dernières pouvant tout autant déboucher sur une captation et une concentration fortes des ressources au profit des seuls riches, que sur une expansion économique et sociale bénéficiant en cascades à toute la population et en premier lieu aux classes moyennes.

Concernant les catégories sociales intermédiaires, le renouveau économique récent semble pouvoir contribuer au redéploiement des gens du milieu dont les caractéristiques sont probablement partiellement nouvelles. Pour l'heure les études manquent pour décrire avec précision les dynamiques en cours. Deux études récentes sur les classes moyennes coordonnées par CFAO (2015) et UCT-UNILEVER (2017) et conduites par IPSOS, ont intégré dans leur panel de pays la Côte d'Ivoire (plus spécifiquement Abidjan). Les résultats de ces études tendent à montrer que ces groupes intermédiaires semblent composés essentiellement de professionnels du public et du privé mais avec proportionnellement plus de salariés du secteur privé que du secteur public et plus de petits et moyens entrepreneurs. Selon l'étude UCT-UNILEVER (2017), les indépendants représentent 38\% de la classe moyenne abidjanaise. De plus, près de $87 \%$ de cette classe moyenne déclare disposer de plusieurs sources de revenus. Dans l'ensemble, cette étude remet un peu en cause l'idée d'une large classe moyenne urbaine disposant de niveaux de vie très 
confortable. Elle tend au contraire à pointer des éléments de vulnérabilité et de précarité. Concernant les processus de différenciation sociale, ces derniers restent probablement fondés sur des bases similaires à celles de la période 1960-1980. Pour les classes moyennes l'enrichissement passe par la captation de revenus provenant de différentes sources (ménages à pluri producteurs de revenus et multi activités des producteurs de revenus) et par des investissements variant avec les capacités. En Côte d'Ivoire, au-delà des formes classiques d'investissement dans de petites entreprises individuelles (transport, commerce...), les catégories moyennes et supérieures des classes moyennes (cadres moyens du public, de l'armée et du privé) investissent également dans le foncier rural soulignant à quel point la culture agricole est centrale dans les processus de développement et demeure un enjeu économique et statutaire important en RCI (Tarouth et Colin, 2016 ; Colin et Tarouth, 2017).

Les processus d'émergence des classes moyennes en Côte d'Ivoire sont donc à mettre en regard des dynamiques de croissance et de stratification sociale de longue période que nous venons d'expliciter. Il s'agit maintenant de travailler à l'identification et la caractérisation d'une ou plusieurs classes moyennes ivoiriennes dans une démarche méthodologique originale combinant les données quantitatives de l'enquête ENV 2015 et des données qualitatives issues d'entretiens auprès des ménages.

\section{A la recherche de(s) la classe(s) moyenne(s) ivoirienne(s) : des gens du milieu de la distribution des revenus}

L'objectif de ce second chapitre est à la fois d'identifier et de caractériser les classes moyennes ivoiriennes dans leur diversité. Ce travail repose sur une méthodologie originale combinant des méthodes quantitatives et qualitatives. Concernant les aspects quantitatifs, les données mobilisées sont celles issues de l'enquête ENV (Enquête Niveau de Vie des ménages) conduite par l'INS (Institut National de la Statistique) en $2015^{11}$. Ces données quantitatives sont utilisées pour délimiter une classe moyenne de revenu au sein de la population, révéler son hétérogénéité en distinguant différents sous-groupes homogènes et en identifier les principales caractéristiques. La méthode adoptée est séquentielle et suit, dans les grandes lignes, la démarche adoptée par Bonnefond et al. (2015). Elle combine une approche économique basée sur le revenu et une approche sociologique mobilisant des informations sur l'emploi et l'éducation, deux dimensions centrales pour caractériser plus qualitativement les classes moyennes (Thurlow et al., 2015). Les données qualitatives sont issues d'entretiens semi-dirigés conduits sur les périodes de mai-juin et octobre-novembre 2017 auprès d'un échantillon de ménages représentatifs des classes moyennes identifiées lors de l'analyse quantitative (cf. encadré $\left.\mathrm{n}^{\circ} 1\right)^{12}$. Les données qualitatives ainsi récoltées permettent alors à la fois de caractériser des éléments transversaux commun à l'ensemble de la classe moyenne mais aussi d'approfondir la compréhension de la dynamique des différents

11 Les données dont nous disposons plus précisément ne couvrent pas l'ensemble des variables disponibles dans la base données ENV 2015 mais seulement celles indispensables à notre analyse.

12 Les entretiens qualitatifs ont été conduits par l'ENSEA sur la période de mai-juin 2017 et par A. Bekelynck (PAC-CI, site ANRS de Côte d'Ivoire - Chercheure associée au CEPED - Université Paris Descartes/IRD) sur la période d'octobre-novembre 2017. 
groupes de classe moyenne ainsi que de leurs principales aspirations. Après être revenu sur les enjeux de l'identification empirique des classes moyennes en Côte d'Ivoire (2.1.), nous présentons notre méthodologie et nos résultats aux regard de l'identification des "gens du milieu" de la distribution des revenus (2.2.). Enfin, nous rentrons plus spécifiquement dans l'analyse de l'hétérogénéité de cette classe moyenne en identifiant et caractérisant les sous-groupes homogènes qui la composent (2.3.).

\subsection{Les dynamiques discontnues du processus de stratification sociale en Côte d'Ivoire : les classes moyennes, un espoir déçu plus qu'une classe constitué}

\subsubsection{Parler de classe moyenne en Côte d'Ivoire : l'invention d'une classe moyenne par évocation}

La Côte d'Ivoire comme la quasi-totalité des pays d'Afrique subsaharienne (exception faite de l'Afrique du sud), se trouve dans une situation paradoxale: la littérature scientifique consacrée à la classe moyenne est finalement assez réduite et ne place pas du tout ce thème au cœur des analyses de la société ivoirienne. L'utilisation non discutée du terme classe moyenne (par exemple Le Pape, 1997) ou sa non utilisation systématique et tout aussi non discutée (par exemple Fauré et Labazée, 2000) témoignent jusque dans les années 2010 de son rejet dans la littérature scientifique dédiée aux sociétés africaines. L'inscription de cette problématique de l'émergence des classes moyennes sur l'agenda public et politique est réalisée au début des années 2010 par les organisations internationales et plus particulièrement la BAD (2011) et la Banque Mondiale et est relayée et amplifiée par des travaux de cabinets internationaux qui se succéderont pour affirmer que "lions are on the move » (MacKinsey Global Institute ; enquêtes IPSOS-Bearing point pour la CFAO en Côte d'Ivoire...) et qu'en dépit d'un début de retournement de conjoncture en Afrique ils sont "(still) on the move» (pour reprendre l'expression du rapport McKinsey\&Company de 2017). Les magazines économiques, les sociétés de marketing et d'études de marché et de consultance, ont emboité le pas à cette innovation et ont fait de ce thème de l'émergence irrésistible des classes moyennes un enjeu central et incontournable érigé en « buzzword » depuis les années 2010. Ainsi le rapport PWC (2015) sur les "continent's cities of opportunity » fait d'Abidjan la ville leader en Afrique pour la croissance des classes moyennes, sans il est vrai vraiment expliquer le mode de calcul permettant d'arriver à cette conclusion.

Les publications des sociétés de consultance internationales et de la $\mathrm{BAD}$ présentent deux intérêts principaux. D'une part, elles permettent de comprendre que la notion de classe moyenne est définie avant tout par des niveaux de revenus et des capacités de consommation de biens et services (le dernier rapport McKinsey\&Company de 2017 parle d'ailleurs de "consumer class » plutôt que de "middle class») Au point que le critère déterminant de leur existence et de leur différenciation d'avec les autres catégories sociales et notamment les pauvres, est avant tout cette capacité de consommation. D'autre part, le principal intérêt de la publication de la BAD (2010) qui va lancer le débat en Afriques (sur des bases aussi fragiles que discutables) est d'avoir attiré l'attention sur les transformations sociales massives en cours sur le continent et notamment sur des mouvements de sortie de pauvreté. Une lecture attentive des chiffres de la BAD permet de voir que ce qui se joue n'est pas tant l'émergence des classes moyennes mais des mouvements ou 
dynamiques confus de sortie de pauvreté à la marge d'une masse d'individus tentant de saisir des opportunités fragiles et constituant la «floating class» ou des ilots remontant ou descendant de l'échelle de la petite prospérité (graphique $\mathrm{n}^{\circ}$ 8). Ces éléments soulignent déjà la grande fluidité d'une catégorie aux contours incertains et aux significations improbables.

\section{Graphique $n^{\circ} 8$ - Une évolution de la classe moyenne en Afrique subsaharienne portée essentiellement par la floating class (classification de la BAD)}

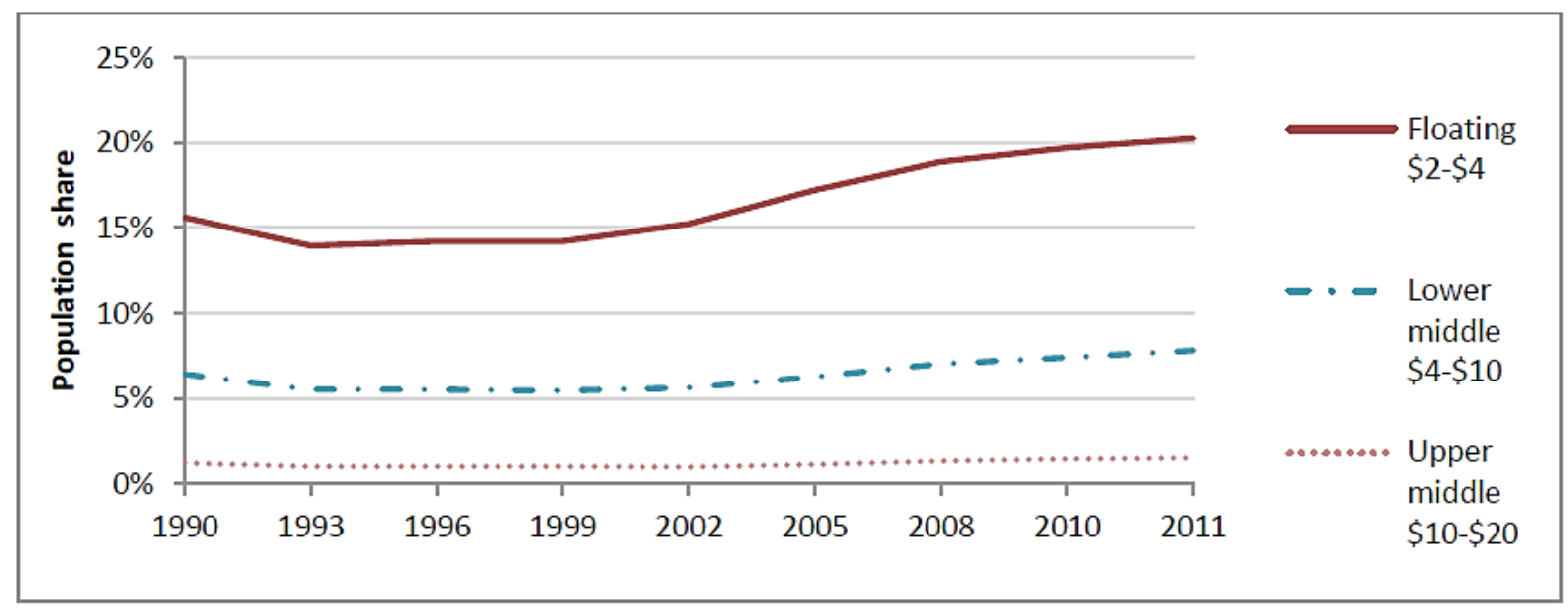

Source: Giesbert and Schotte (2016, p.4), PovCal data 2011

\subsubsection{L'identification des mutations sociales structurelles en cours en Côte d'Ivoire ou comment mesurer les classes moyennes.}

Les plans nationaux de développement (PND 2012-2015 et 2016-2020), identifiant les conditions de l'émergence ivoirienne, ne donnent qu'une importance très relative à la notion de classe moyenne. L'expression «classe moyenne » n'est d'ailleurs utilisée qu'une fois dans le PND 20162020, au sein du discours introductif du président Ouattara. L'emphase sur les classes moyennes en RCI depuis 2011 ne semble donc pas à l'évidence provenir de leur existence démontrée ou du poids de leur capacité économique ou politique, mais bel et bien d'un effet du buzz « african middle class » localement repris et validé à partir des critères et marqueurs locaux ${ }^{13}$. La déconstruction de la fabrication des classes moyennes ivoiriennes conduit à s'intéresser à un ensemble d'indices qui soulignent des mutations socioéconomiques profondes des structures économiques et sociales de la Côte d'Ivoire, à partir desquels il devient possible de commencer à dresser les contours d'une éventuelle classe moyenne. Comme le souligne le rapport CFAO (2015), se côtoient dans cette perspective deux grandes approches : l'une porte sur des indices traduisant des comportements spécifiques, avec une attention particulièrement poussée sur les comportement de consommation, l'autre porte sur une identification des classes moyennes par les niveaux de revenus ${ }^{14}$.

\footnotetext{
${ }^{13}$ Avec quelques réserves comme le montre le rapport CFAO (2015).

14 Les approches «comportementales» s'intéressent également aux avoirs patrimoniaux (actifs possédés) (cf. Shimeless et Ncube, 2015 ; Johnston et Abreu, 2016 ; Schotte, 2017).
} 
La notion de classes moyennes en Afrique telle qu'elle est construite dans les magazines économiques est essentiellement associée à celle de progression du marché et de la consommation intérieure. Dans le rapport Lions (still) on the move de McKinsey \& Company (2017), la notion est directement liée à celle d'un marché de la consommation se montant à 1,4 trillion US\$ en Afrique concentré pour plus de 50\% sur trois pays : Nigeria, Egypte, Afrique du sud. Or cette présentation occulte derrière l'image unique d'un modèle de consommateurs aisés, urbains, éduqués et occidentalisés, un ensemble de phénomènes de croissance de la consommation extrêmement disparates. Trois phénomènes différents se cachent derrière l'expansion du marché de la consommation intérieure privée.

Il y a d'abord un mouvement de croissance urbaine qui maille le territoire et concentre à la fois les compétences, les consommations, les investissements et les besoins. Douze villes ont désormais plus de 100000 habitants (dont Divo, Soubré, Abengourou) drainant autour d'elles une partie de la population périurbaine. Le district d'Abidjan (en raison du poids de la ville d'Abidjan) avec ses 4707404 habitants abrite le cinquième de la population totale (20,8\%) et est classé numéro un en Afrique pour la qualité et la densité de ses «classes moyennes par PWC (2015). Ce mouvement d'urbanisation accompagne une expansion démographique (cf. chapitre 1) qui ne cesse d'augmenter mécaniquement les besoins mais aussi les dépenses de consommation et notamment dans les zones urbaines. Pour autant, rien ne garantit le prolongement dans le temps de cette capacité de consommation menacée d'une part par les coûts d'infrastructures considérables que génère un processus d'urbanisation rapide et d'autre part par l'absence de relai de ce moteur consommation par des capacités locales urbaines de production massive de valeur ajoutée (notamment manufacturière). Il y a ici un enjeu considérable que l'aporie du catéchisme causaliste voyant dans les classes moyennes des vecteurs naturels de l'entreprise privée ne peut suffire à traiter.

Ensuite, on assiste à l'expansion de la consommation d'un ensemble de consommateurs très aisés ou disposant de revenus élevés et constants. Dans la littérature classique et les travaux des instituts de marketing, l'essentiel de la preuve de l'existence de ces classes moyennes en Afrique est véhiculé par: (i) l'implantation de malls et des centres commerciaux; (ii) l'expansion des marchés immobiliers urbains et l'augmentation de prix des locations et (iii) l'amélioration des taux d'équipements des ménages en électro ménagers, automobiles etc. Ce type d'approche est risqué dans la mesure où ces types de consommation peuvent se révéler trompeurs. Ils présentent comme formes quotidiennes de la consommation des classes moyennes ce qui ne l'est pas. L'usage des malls pour les achats du quotidien ne concerne probablement qu'un nombre limité de ménages, ceux disposant déjà d'un niveau de revenus substantiels et qu'on peut qualifier de riches. Il s'agit très vraisemblablement d'une minorité de la population, la plus aisée, qui concentre la plus grande partie du revenu global et dont les comportements de consommation ressemblent à ceux des classes moyennes aisées occidentales mais qui ne constituent en aucun cas des classes moyennes au sens occidental du terme et ne forment en aucun cas le centre de la toupie ou du diamant de la distribution des revenus pour reprendre les métaphores consacrées. 
Localement, ces foyers font partie de la catégorie des riches allant au mieux jusqu'à la Upper middle class, mais jamais en deçà. Ponctuellement des foyers moins fortunés peuvent réaliser des achats spécifiques dans ces «temples de la consommation à l'occidental », tandis que le pari à terme des investisseurs de la grande distribution est d'optimiser leur investissement au moment de l'arrivée à maturation du marché à 10 ans des groupes de ménages à faibles revenus mais en voie d'enrichissement (CFAO, 2015, McKinsey\&Company, 2017)

Enfin, l'augmentation de la consommation provient aussi de la masse considérable des foyers moins fortunés mais en flux constant, oscillant constamment entre précarité et petite prospérité. Cette approche permet de prendre en compte les travaux portant sur l'émergence de nouveaux marchés de la consommation concernant toutes les catégories de la population y compris celles caractérisées par les marchés dits « BoP» ou bas de la pyramide (Prahalad, 2004). Peut-on définir ces foyers comme constitutifs de classes moyennes ? Le rapport McKinsey \& Company (2017) identifie pour quelques pays d'Afrique centrale et occidentale dont la Côte d'Ivoire les foyers dits " emerging consumers 》 dont les revenus annuels sont compris entre 5000 et 20.000 US\$ (responsables de 40\% de la croissance de la consommation d'ici 2025) et les foyers dits "global consumers » aux revenus annuels compris entre 20.000 et 50.000 US\$ (responsable pour $33 \%$ de la croissance de la consommation d'ici 2025). On voit ainsi poindre le poids de ces foyers en dynamiques, en sortie tendancielle de pauvreté et qui pourraient peut-être bien être en 2025 le gros des « classes moyennes » ivoiriennes.

Approcher les classes moyennes par leur niveau de vie : quid de la floating class?

En Côte d'Ivoire, comme dans la plupart des pays d'Afrique subsaharienne (hors Afrique du Sud), parler de classes moyennes suppose d'essayer de concilier un critère de masse de population avec un niveau significatif de revenu. Ceci s'avère particulièrement complexe, compte tenu du bas niveau de revenu de la population en général et du très fort niveau de pauvreté. Comme le remarquent nombre de travaux, il est particulièrement difficile de cibler des classes moyennes lorsque le revenu médian de la population est inférieur au seuil de pauvreté et lorsque l'application des normes utilisées pour les autres pays en développement hors Afrique, conduirait à réduire de manière considérable le groupe classe moyenne. Entre les «en-haut des en-bas, les en-bas des en-haut " pour reprendre l'expression de Savina et al. (1985), les ruptures ne sont pas franches et la démarcation d'autant plus complexe que la différenciation par les statuts, par les types d'emplois, et le salariat par exemple, ne fonctionnent qu'à la marge (sauf exceptions comme en Afrique du Sud ; cf. Visagie et posel, 2013). La classification des classes moyennes par les revenus est ainsi extrêmement périlleuse. Soit on tente d'englober un nombre élevé de la population mais au risque de descendre très bas en termes de revenus et de constituer des groupes statistiques d'une très grande hétérogénéité de milieux ; soit on privilégie une cohérence de groupes de revenus et alors le groupe classe moyenne apparaitra très réduit.

Le rapport de la BAD (2011) fixant les bornes inférieure et supérieur de la classe moyenne africaine respectivement à 2 et à $20 \$$ par tête et par jour, adopte finalement une curieuse position consistant à différencier plusieurs groupes pour in fine assimiler sans vraiment l'affirmer la floatting 
class à la classe moyenne pourtant constituée en groupes spécifique... (Figure $\mathrm{n}^{\circ} 2$ 2) $^{15}$. Les résultats de cette étude conduisent à évaluer à 37,1\% la part de la population relevant de la classe moyenne en Côte d'Ivoire en 2011 (la floating class représentant 18,9\% de la population). Une étude plus récente des mêmes auteurs avec des données actualisées conduit à modifier ces résultats puisque la classe moyenne ivoirienne y est estimée pour la même année 2010 à $55 \%$ de la population et $35 \%$ pour la floating class (Ncube et Lufumpa, 2015). Ainsi, plus de $60 \%$ de la classe moyenne relève de la floating class et la Upper middle class n'en représente qu'à peine $5 \%$.

Pour sortir de ce dilemme le rapport CFAO (2015) sur les classes moyennes en Afrique et notamment en Côte d'Ivoire, identifie une classe moyenne allant d'un revenu de 2,6 US\$ par tête et par jour à 10,5 US\$ (revenu moyen de 4,8US\$). Puis il se focalise sur la partie hors floating class (donc au-delà de la catégorie de revenu de 2 à 4 US\$) pour s'intéresser à une classe moyenne de 1,8 millions de personnes «effectivement consommatrices » à laquelle on rajoute 2 millions d'enfants de moins de 18 ans et constituant 19\% de la population. La classe flottante est donc partiellement intégrée à l'origine du raisonnement (dans la lignée des travaux de la $\mathrm{BAD}$ ) pour finalement être ignorée car trop fragile en termes de capacités de consommation. L'étude CFAO cible en réalité un potentiel de consommation solvable et des classes moyennes enrichies (stabilisées) plus que la totalité des catégories sociales émergentes et notamment celles oscillant entre pauvreté et petite prospérité (conservées en potentiel de marché en maturation).

Figure $\mathrm{n}^{\circ} 2$ - Part de la classe moyenne dans la population totale en Afrique selon la BAD

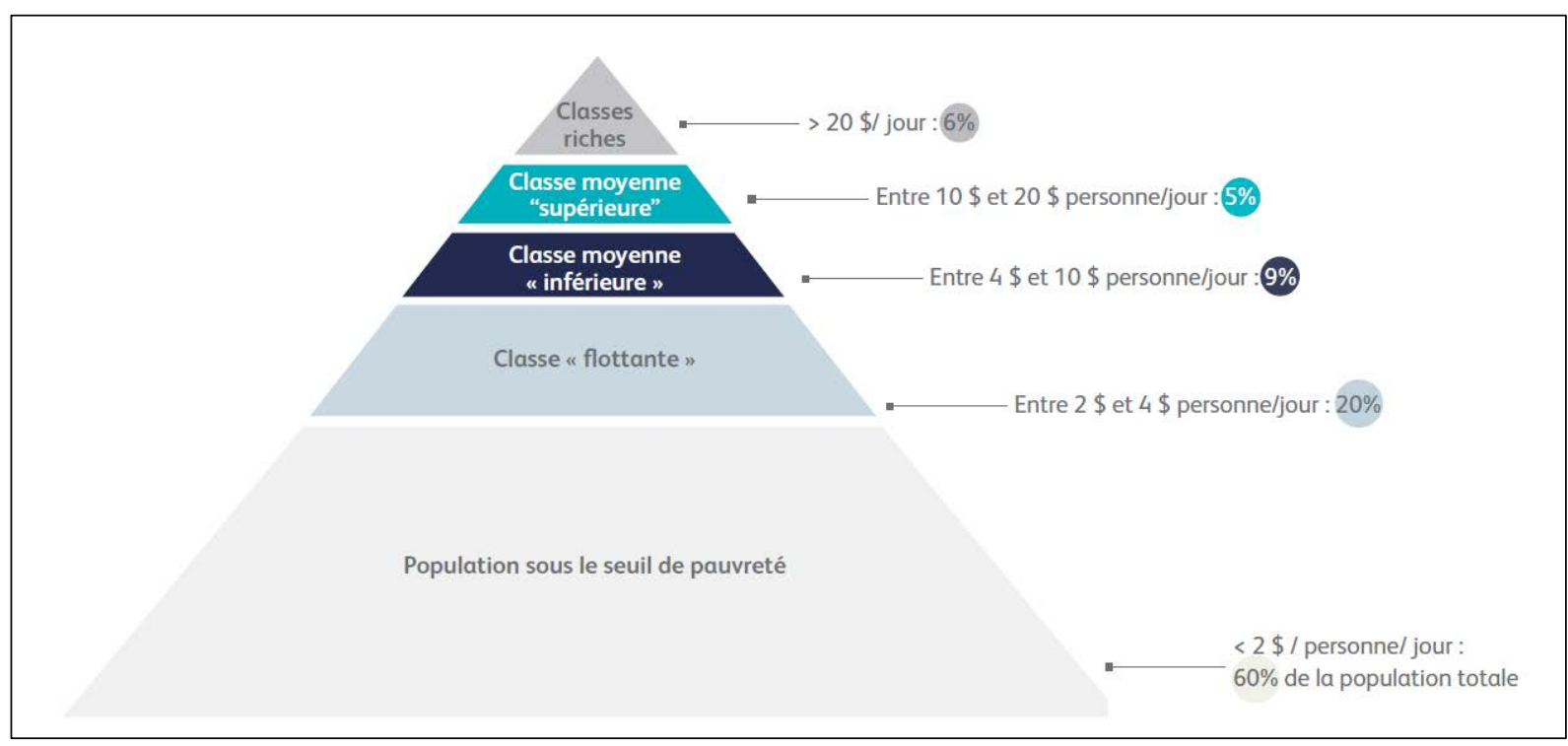

Source : Rapport CFAO (2015, p.9)

Cette approche par les revenus présente ainsi un problème majeur, celui de favoriser une confusion des « milieux » en rassemblant des types de foyers radicalement dissemblables en termes de revenus. A certains égards notre caractérisation quantitative n'est pas exempte de cette

15 Pour être exacte, la BAD retient dans cette étude comme mesure du niveau de vie la dépense de consommation par tête. 
critique ; mais d'une part c'est la condition pour délimiter un niveau de population significatif de ce qu'on appelle généralement classe moyenne (cf. 2.2.); et d'autre part nous accompagnons ce critère de revenu de critères plus qualitatifs relatifs à l'éducation et au statut d'emploi afin d'identifier des sous-catégories pouvant faire l'objet d'interprétations socioéconomiques et historiques (cf. 2.3.).

\subsection{Identifier et caractériser la classe moyenne ivoirienne : les gens du milieu en Côte d'Ivoire, une catégorie limitée de la population}

L'identification statistique des classes moyennes en Côte d'Ivoire se fonde sur une analyse quantitative des données d'enquête ENV (Enquête Niveau de Vie des ménages) collectées par l'INS (Institut National de la Statistique). L'année d'enquête de référence est 2015 et l'enquête couvre approximativement 13000 ménages. Notre but à ce stade de l'analyse est d'abord de délimiter une classe moyenne de revenu. Il ne s'agit pas d'identifier précisément une taille de classe moyenne (compte tenu de l'absence de consensus sur le choix de l'intervalle monétaire), mais plutôt de délimiter un ensemble d'individus situés au milieu de la distribution du revenu (i.e. les « gens du milieu »). Deux étapes structurent notre méthodologie ici. La première repose sur l'analyse de la distribution du revenu dans le pays afin de permettre de donner des "guides » pour le choix d'un intervalle monétaire pertinent. La seconde vise à l'identification et la caractérisation de la classe moyenne à partir du choix d'un critère monétaires pertinent et permettant la comparaison entre pays.

\subsubsection{La délimitation d'une classe moyenne revenu}

La première étape consiste à examiner la distribution des revenus en Côte d'Ivoire. Les outils privilégiés lors de cette étape sont l'estimation de fonctions kernel (permettant de reconstituer la distribution du revenu de manière continue) et l'estimation des différents seuils de pauvreté et d'inégalité habituels. L'indicateur de revenu est le revenu des ménages par tête et intègre toutes les composantes habituelles du revenu (revenus du travail formel et informel, revenus du patrimoine, transferts publics et privés, etc. $)^{16}$.

16 Nous avons retenu une mesure du niveau de vie par le revenu plutôt que par la dépense de consommation en raison de la dimension comparative du projet dans lequel s'inscrit cette étude (comparaison avec le Brésil, la Turquie et le Vietnam). Nous sommes évidemment conscients que cette démarche méthodologique dans le cas d'une économie africaine peut poser question compte tenu des difficultés connues à mesurer correctement les revenus (importance des revenus informels, instabilité des revenus...). Une grande partie des études utilisent ainsi plutôt la dépense de consommation comme mesure du niveau de vie. On sait que pour des niveaux bas de revenus, la différence entre dépense de consommation et revenu est très faible mais que cette différence s'accroit en revanche avec l'augmentation du revenu... De fait, notre approche par le revenu nous expose à un risque de de sousestimer le poids de la floating class. En revanche, il est fort probable que l'on mesure mieux la Upper middle class... En terme d'impact de ce choix méthodologique sur l'effet de structure et de composition de la classe moyenne, les résultats présentés plus bas semblent suggérer qu'ils sont peu probables au regard de la diversité des situations ouvertes avec nos bornes de revenus. 
La figure $\mathrm{n}^{\circ} 3$ suivante représente la distribution des revenus par tête mensuels des ménages ivoiriens en 2015, ainsi que la position sur celle-ci de plusieurs seuils utiles à l'identification de la classe moyenne de revenus. On le remarque nettement, cette distribution est extrêmement oblique à gauche du fait du haut niveau de la pauvreté et des inégalités de revenu. On note ainsi une concentration de la population à des niveaux de revenus globalement bas. Sur la base de cette distribution des revenus, nous mesurons la taille de la classe moyenne à partir d'un ensemble de treize intervalles utilisés dans littérature et regroupes en trois ensembles (approche absolue, relative ou mixte) ${ }^{17}$. Les poids des différents groupes (pauvres, classe moyenne, riches) pour la Côte d'Ivoire pour ces 13 intervalles différents sont reportés dans le tableau n³. On observe que les différents critères retenus produisent des résultats assez disparates en termes de poids de la classe moyenne celui-ci étant compris entre $1 \%$ et $46 \%$ de l'ensemble de la population selon le critère retenu pour l'année 2015. Ceci s'explique par la diversité des intervalles qui, dans de nombreux cas, ne se recoupent que très partiellement, voire pas du tout. Ainsi l'intervalle $2 \$-10 \$$ ne recoupe aucun des intervalles démarrant à $10 \$$. Concernant l'approche absolue (les six premiers intervalles), on notera que le choix d'une borne inférieure à 2 ou $4 \$$ conduit à modifier considérablement le poids de la classe moyenne (de l'ordre de 20 points de pourcentage), alors même que le choix d'une borne supérieure à 10 ou 20\$ a quant à lui moins d'impact (de l'ordre de 7 points de pourcentage). Cela traduit bien l'idée que le «gonflement» du poids de la classe moyenne dépend pour principalement de l'inclusion ou non de cette fameuse «floating class » (BAD, 2011 ; Darbon, 2012). Toujours dans l'approche absolue, l'ensemble des intervalles avec une borne inférieure à 10 , souvent retenus pour étudier les pays émergents (comme le Brésil par exemple), conduisent à un poids extrêmement réduit le classe moyenne ivoirienne (autour de 9-10 $\%$ de la population). Concernant les approches relatives fixant un intervalle autour de la médiane, elles sont ici problématiques dans la mesure où la médiane de la distribution du revenu de la population est quasiment au niveau du seuil de pauvreté. Enfin, au niveau des approches mixtes, il s'agit d'exclure les $5 \%$ ou les $10 \%$ les plus riches de la population et de fixer un seuil absolu comme borne inférieure. Les quatre seuils présentés dans le tableau $\mathrm{n}^{\circ} 3$ conduisent à des résultats assez disparates.

17 Il importe de préciser que nous privilégions des intervalles monétaires utilisés dans la littérature comparative (qu'il s'agisse d'une approche absolue, relative ou mixte). En effet, cette étude sur la Côte d'ivoire s'insère dans un projet de recherche plus large examinant l'essor des classes moyennes et ses implications dans quatre pays distincts (le Brésil, la Turquie, le Vietnam et donc la Côte d'Ivoire). Parmi les treize critères sélectionnés, on notera que seul celui de la BAD (2\$-20\$) peut être considéré comme spécifique au terrain étudié. 
Figure $n^{\circ} 3$ - Distribution des revenus mensuels per capita en FCFA (Côte d'Ivoire, 2015)

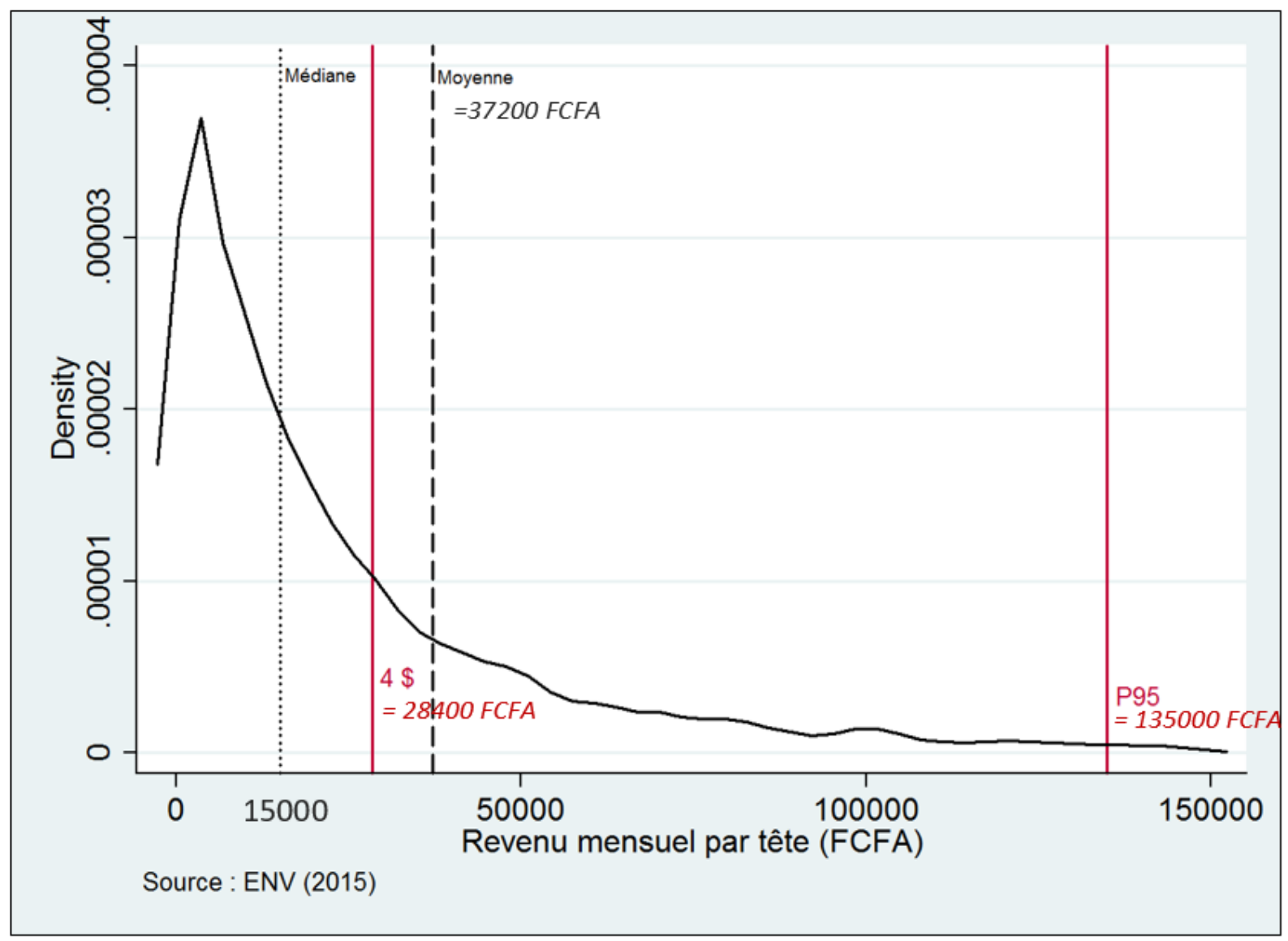

Nous considérons pour notre part comme impératif de sélectionner une borne inférieure ni trop basse lau risque de capter des ménages plus "pauvres » que "classes moyennes », cf. le débat sur la "floatting class »), ni trop baute (afin de pouvoir intégrer des ménages non pawres mais qui resteraient potentiellement vulnérables à un retour dans la pauvreté). L'ensemble de ces éléments nous amènent ainsi à retenir le seuil de 4.00 \$ par jour comme borne inférieure de la classe moyenne de revenu ivoirienne. On s'éloigne ainsi clairement du seuil de pauvreté de deux dollars par jour, qui concerne près de la moitié de la population, tout en conservant une part suffisamment conséquente de la population totale allant jusqu'au percentile 95 (P95) (les 5\% les plus riches, dont les revenus sont essentiellement composés de revenus du patrimoine) ${ }^{18}$.

18 Notons que cette borne supérieure fixée au percentile 95, soit un revenu mensuel par tête d'environ 135000 FCFA, ne doit pas faire oublier l'extrême dispersion des revenus parmi ces $5 \%$ les plus riches. Le seuil d'entrée dans les 1\% les plus riches est ainsi de presque 2000000 FCFA mensuel tête. Les travaux de Czajka et Cogneau (2016), centrés sur le secteur formel (public et privé) vont dans ce sens. A partir des données ENV 2015, ils trouvent un seuil d'entrée dans les 10\% les plus riches du formel d'approximativement 400000 FCFA à mettre en comparaison avec le seuil d'entrée des $1 \%$ et $0,1 \%$ les plus riches d'environ respectivement plus de 2000000 FCFA et plus de 8000000 FCFA. Ils montrent de plus que les données d'enquête ménages mesurent assez mal et sous estiment les revenus des plus riches en comparaison des données fiscales (elles même sous-estimées). 
Ramenées en FCFA, nos bornes inférieures et supérieures de la classe moyenne sont donc respectivement d'environ 28400 FCFA et 135000 FCFA par tête et par mois ${ }^{19}$. Des bornes pas si éloignées de celles de la catégorie «emerging consumer» de McKinsey et Company (2017). La grande différence avec l'intervalle de la BAD (2011) se situe quant à elle au niveau du choix de la borne inférieure et l'exclusion de la floating class.

Tableau n³ - Comparaison de différents critères monétaires pour définir la classe moyenne (Côte d'Ivoire, 2015)

\begin{tabular}{|c|c|c|c|c|c|}
\hline \multirow[t]{2}{*}{ Critères } & \multicolumn{2}{|c|}{ Intervalles de revenu } & \multirow{2}{*}{$\begin{array}{c}\text { Pauvres } \\
(\%)\end{array}$} & \multirow{2}{*}{$\begin{array}{c}\text { Classe moyenne } \\
(\%)\end{array}$} & \multirow{2}{*}{$\begin{array}{l}\text { Riches } \\
(\%)\end{array}$} \\
\hline & $\begin{array}{l}\text { FCFA mensuels par tête } \\
\text { (prix 2015) }\end{array}$ & $\begin{array}{l}\text { \$ mensuels par tête } \\
\text { (PPA 2011, prix 2015) }\end{array}$ & & & \\
\hline$[2 \$ ; 10 \$]$ & [14 198; 70 992] & {$[60 \$ ; 300 \$]$} & 49,1 & 39,2 & 11,7 \\
\hline$[2 \$ ; 20 \$]$ & [14 198; 141985$]$ & {$[60 \$ ; 600 \$]$} & 49,1 & 46,2 & 4,7 \\
\hline$[4 \$ ; 20 \$]$ & [28 396; 141985$]$ & {$[120 \$ ; 600 \$]$} & 68,6 & 26,7 & 4,7 \\
\hline$[10 \$ ; 20 \$]$ & {$\left[\begin{array}{lllll}70 & 992 ; 141 & 985\end{array}\right]$} & {$[300 \$ ; 600 \$]$} & 88,3 & 7,0 & 4,7 \\
\hline$[10 \$ ; 50 \$]$ & {$\left[\begin{array}{llll}70 & 992 ; & 354 & 962\end{array}\right]$} & {$[300 \$, 1500 \$]$} & 88,3 & 10,8 & 0,9 \\
\hline$[10 \$ ; 100 \$]$ & [70 992; 709 925] & {$[300 \$ ; 3000 \$]$} & 88,3 & 11,4 & 0,3 \\
\hline $\begin{array}{c}\text { [75\%- } 125 \% \text { revenu } \\
\text { médian] } \\
{[50 \%-150 \% \text { revenu }}\end{array}$ & {$[11250 ; 18$ 750] } & [47 \$; 79\$] & 43,0 & 13,8 & 43,2 \\
\hline & [7 500; 22500$]$ & {$[32 \$ ; 95 \$]$} & 34,0 & 28,1 & 37,9 \\
\hline moyen] & {$\left[\begin{array}{lllll}37 & 206 ; & 93 & 015\end{array}\right]$} & {$[157 \$ ; 393 \$]$} & 75,2 & 16,5 & 8,3 \\
\hline$[10 \$ ; \mathrm{P} 90]$ & {$\left[\begin{array}{llll}70 & 992 ; 80 & 000\end{array}\right]$} & {$[300 \$ ; 338 \$]$} & 88,3 & 1,4 & 10,3 \\
\hline [10\$; P95] & {$\left[\begin{array}{llll}70 & 992 ; 135 & 000\end{array}\right]$} & [300\$; 570\$] & 88,3 & 6,7 & 5,0 \\
\hline [4\$; P95] & [28 396; 135000$]$ & {$[120 \$ ; 570 \$]$} & 68,6 & 26,4 & 5,0 \\
\hline [2\$; P95] & {$[14198 ; 135000]$} & {$[60 \$ ; 570 \$]$} & 49,1 & 45,9 & 5,0 \\
\hline
\end{tabular}

Note : Facteur de conversion PPA (PPA 2011 ajusté aux prix 2015)=236,641 (Source : Banque Mondiale). Source : données ENV (2015).

Ainsi calibrée, la classe moyenne monétaire ivoirienne, «les gens du milieu » de la distribution des revenus, représente finalement une masse relativement limitée de $26,4 \%$ de la population $(68,6 \%$ de la population à moins de $4 \$$ et $5 \%$ de riches).

\subsubsection{Brève caractérisation de la classe moyenne de revenus}

Si les gens du milieu de la distribution des revenus représentent une catégorie limitée de la population ivoirienne, ils présentent en revanche une large gamme de revenus et de situations monétaires allant probablement d'un niveau de vulnérabilité élevé à un niveau d'aisance important et laissant entrevoir la grande hétérogénéité des «milieux » ainsi associés. Cette première lecture remet ainsi potentiellement en cause l'idée sous-jacente d'une classe

19 Considérant que la taille moyenne des ménages de la classe moyenne est de 3 personnes (tableau 6), cela donne un revenu par ménage compris entre approximativement entre 85200 et 405000 FCFA. 
moyenne disposant d'une aisance limitée mais certaine et conteste l'idée d'une classe moyenne rassemblant dans cette aisance une majorité de la population.

Le tableau $\mathrm{n}^{\circ} 4$ nous permet de caractériser les trois groupes initialement identifiés sur la base de ce critère de revenu, celui des « pauvres », celui de la « classe moyenne » et celui des « riches », par les différentes variables de classification retenues. Ces variables, dites de classification, sont celles qui nous permettront dans un second temps de mieux appréhender la diversité de cette classe moyenne " monétaire » en considérant simultanément les statuts occupationnels et éducatifs des ménages. Sans rentrer dans les détails, ce tableau nous donne quelques éléments de caractérisation intéressant. En termes d'éducation, 27,6\% des chefs de ménage de cette classe moyenne de revenu dispose d'un niveau d'éducation supérieur, une proportion similaire à celle que l'on retrouve chez les riches pour ce niveau d'éducation. En revanche, seuls 7,8\% des chefs de ménage des gens du milieu dispose d'un

Tableau $\mathrm{n}^{\circ} 4$ - Caractéristiques (variables de classification) des différents groupes (pauvres, classe moyenne, riche). Côte d'Ivoire, 2015

\begin{tabular}{|c|c|c|c|c|}
\hline & Pauvres & $\begin{array}{c}\text { Classe } \\
\text { moyenne }\end{array}$ & Riches & Ensemble \\
\hline \multicolumn{5}{|l|}{ Education } \\
\hline Sans éducation & 66,0 & 48,1 & 33,4 & 59,6 \\
\hline Education primaire & 16,5 & 16,6 & 13,1 & 16,3 \\
\hline Education secondaire & 16,2 & 27,6 & 27,5 & 19,8 \\
\hline Education supérieure & 1,4 & 7,8 & 26,1 & 4,3 \\
\hline \multicolumn{5}{|l|}{ Catégorie socioprofessionnelle } \\
\hline Inactifs et chômeurs & 38,7 & 15,2 & 11,4 & 31,2 \\
\hline $\begin{array}{l}\text { Dirigeants, cadres supérieurs, professions } \\
\text { intellectuelles }\end{array}$ & 0,8 & 4,3 & 13,4 & 2,4 \\
\hline Professions intermédiaires & 3,1 & 12,1 & 15,8 & 6,1 \\
\hline Employés et travailleurs des services & 10,9 & 20,6 & 22,9 & 14,0 \\
\hline Ouvriers & 13,9 & 21,0 & 15,2 & 15,8 \\
\hline Agriculteurs & 32,6 & 26,9 & 21,3 & 30,5 \\
\hline \multicolumn{5}{|l|}{ Statut dans l'emploi } \\
\hline Inactifs et chômeurs & 37,6 & 14,5 & 11,0 & 30,2 \\
\hline Salariés, employés & 15,5 & 38,1 & 48,3 & 23,1 \\
\hline Indépendants & 38,9 & 43,0 & 36,1 & 39,8 \\
\hline Employeurs & 0,4 & 1,1 & 1,9 & 0,6 \\
\hline Travailleurs non rémunérés & 7,7 & 3,3 & 2,8 & 6,3 \\
\hline \multicolumn{5}{|l|}{ Type d'employeur, secteur institutionnel } \\
\hline Inactifs et chômeurs & 37,6 & 14,5 & 11,0 & 30,2 \\
\hline Emploi privé formel & 1,3 & 3,8 & 8,1 & 2,3 \\
\hline Emploi privé informel & 59,0 & 70,6 & 58,1 & 62,0 \\
\hline Emploi public & 2,1 & 11,1 & 22,8 & 5,5 \\
\hline Pluri-activité & & & & \\
\hline
\end{tabular}




\begin{tabular}{lcccc} 
Non & 97,4 & 96,1 & 91,5 & 96,8 \\
Oui & 2,6 & 3,9 & 8,5 & 3,2 \\
\hline
\end{tabular}

Note: La classe moyenne est définie comme les ménages dont le revenu se situe entre $4 \$$ par jour et le 95 ème percentile.

Source : données ENV (2015)

niveau d'éducation supérieure, une proportion bien inférieure à celle des riches (26,1\%). De manière plus frappante, on peut noter que $48,1 \%$ des chefs de ménage de cette classe moyenne est sans éducation. Un résultat qui contraste quelque peu avec les représentations habituelles de la classe moyenne ivoirienne souvent associée à un niveau d'éducation secondaire au minimum (CFAO, 2015. UCT-UNILEVER, 2017). Au regard des caractéristiques d'occupation d'emploi, comparativement au plus pauvre on retrouve au sein de cette classe moyenne une proportion importante de chefs de ménage dans les catégories professions intermédiaires, employés et travailleurs de services et ouvriers. Une proportion moindre d'entre eux se situe dans une position d'inactivité ou de chômage. En termes de statut d'emploi, ces chefs de ménages sont majoritairement indépendants ou salariés-employés, et pour 70,6\% d'entre eux ils exercent dans le secteur informel urbain ou rural (une proportion plus élevée que pour les riches). 11\% d'entre eux sont dans la fonction publique, une proportion deux fois moindre que pour les plus riches. Enfin, notons un résultat surprenant concernant la pratique de la pluriactivité puisqu'elle ne concernerait qu'en moyenne seulement $4 \%$ des chefs de ménages de la classe moyenne ${ }^{20}$. Par ailleurs, les statistiques présentées dans les tableaux 5 et 6 (cf. infra) nous permettent aussi d'apporter quelques éléments de caractérisation complémentaires de ces gens du milieu. Il ressort de ces tableaux que $25 \%$ des chefs de ménages de la classe moyenne sont de nationalité non ivoirienne, une proportion similaire à celle prévalent pour l'ensemble de la population (cf. chapitre 1). Ensuite, on observe que $58 \%$ de la classe moyenne ivoirienne est urbaine avec notamment $16 \%$ de cette dernière résidant à Abidjan. Cette part finalement assez réduite de la capitale dans la répartition spatiale de la classe moyenne ivoirienne traduit peut-être le haut niveau d'inégalité qui caractérise la Abidjan avec une forte concentration des richesses dans le top 5\% des revenus (lui-même très hétérogène) et une masse importante de la population en situation de précarité. Ce sentiment d'exacerbation des inégalités à Abidjan est assez largement confirmé par l'ensemble de nos interviewés lors des entretiens qualitatifs. On remarque d'ailleurs que si en moyenne 46\% des chefs de ménages de la classe moyenne sont propriétaires de leur logement, cette proportion est tirée vers le haut par les classes moyennes rurales alors qu'en milieu urbain on se rapproche du taux de 34\% estimé par UCT-UNILEVER (2017) pour Abidjan. Enfin, on note que près de $85 \%$ des chefs de ménage de la classe moyenne ivoirienne sont des hommes. Les ménages de cette classe moyenne sont en moyenne composés 3 personnes (en tenant compte

20 A ce sujet, nous ne pouvons que constater un écart conséquent entre ces données quantitatives et la réalité décrites pas nos observations qualitatives (cf. infra, encadré ${ }^{\circ} 1$ ) témoignant au contraire de l'importance de la pratique de la pluriactivité (investissements dans l'immobilier, le transport, le foncier et les plantations...). 
de la dispersion on est en moyenne entre une et cinq personnes). Le chef de ménage contribue en moyenne à 90\% du revenu du ménage, un résultat également observé dans l'étude CFAO (2015).

\section{Encadré $n^{\circ} 1$ - Méthodologie des entretiens qualitatifs auprès des ménages}

Les entretiens qualitatifs auprès de ménages ont reposé sur la méthode de l'entretien semidirectif et ont articulé une entrée par le récit à une entrée par les pratiques. En plus de la consigne générale orientant le discours de l'interviewé, une grille de thèmes a été définie. La consigne générale était la suivante : "Nous soubaiterions échanger avec vous et que vous nous racontiez. votre histoire, votre parcours de vie personnelle et professionnelle, vos conditions de vie actuelles, ainsi que vos projets, vos opinions et vos attentes au regard des autorités publiques de votre pays. ». La grille d'entretien par thématiques avait vocation à couvrir des thèmes jugés important dans le cadre du projet et à faciliter la comparaison entre les discours collectés. Dix thématiques ont structuré cette grille : (i) données de cadrage (caractéristiques sociodémographiques) ; (ii) itinéraire/trajectoire personnelle et professionnelle (capital humain, mobilité sociale, satisfaction) ; (iii) emplois, revenus et appartenance sociale (activité(s), perception d'appartenance sociale) ; (iv) comportements financiers et de consommation (épargne, investissement, dépenses et pratiques de consommation) ; (v) conditions de vie (habitat, accès à l'énergie, mobilitéconnectivité) ; (vi) éducation et inclusion au marché du travail (préoccupation en termes de scolarisation, de formation et d'accès à l'emploi); (vii) protection sociale et fiscalité (gestion des risques sociaux, perception de la fiscalité, consentement à payer, attentes vis-à-vis des autorités...) ; (viii) sécurité-démocratie ; (ix) participation à la vie sociale et politique (sociabilité, engagements collectifs, perception du politique) ; (x) projections et priorités (perspectives de dépenses et investissements, priorités collectives, attentes vis-à-vis des autorités publiques). L'objectif de ces entretiens était donc de compléter les informations produites par l'analyse quantitative des données. Il s'agit donc de parvenir à une meilleure compréhension "de l'intérieur » des représentations, attitudes, motivations et pratiques d'un groupe social particulier, la classe moyenne, et ses différentes composantes.

Au total, 31 entretiens ont été menés sur les périodes d'avril-mai et octobre-novembre 2017. La sélection des ménages s'est faite selon la méthode des quotas afin de respecter poids des différents sous-groupes de classes moyennes identifiés dans l'analyse quantitative et leur répartition entre zones urbaine (Abidjan) et rurale (Soubré) (tableau n ${ }^{\circ} 7$ ). Les entretiens ont été conduits par l'ENSEA sur la période de mai-juin 2017 et par A. Bekelynck (PAC-CI, site ANRS de Côte d'Ivoire - Chercheure associée au CEPED - Université Paris Descartes/IRD) sur la période d'octobre-novembre 2017. 
Tableau $n^{\circ} 7$ - Répartition des interviewés par catégories de classe moyenne et zone géographique

\begin{tabular}{|l|c|c|}
\hline Catégorie de Classe Moyenne & Abidjan & Soubré \\
\hline Agriculteurs & 2 & 5 \\
\hline Travailleurs de l'économie informelle & 5 & 2 \\
\hline Classe moyenne intermédiaire formelle & 4 & 1 \\
\hline $\begin{array}{l}\text { Dirigeants, cadres et professions intermédiaires du } \\
\text { secteur public }\end{array}$ & 6 & 1 \\
\hline Retraités et inactifs & 4 & 1 \\
\hline Total (par zone) & $\mathbf{2 1}$ & $\mathbf{1 0}$ \\
\hline Total (général) & \multicolumn{2}{|c|}{$\mathbf{3 1}$} \\
\hline
\end{tabular}

Les entretiens qualitatifs mettent aussi en évidence de manière transversale que ces catégories intermédiaires de la population se caractérisent par un effort d'épargne assez conséquent ce qui les distingue assez nettement des catégories les plus pauvres. En moyenne sur l'ensemble des entretiens réalisés, cet effort d'épargne dans circuits formels comme informels se situe à hauteur de $20 \%$ du revenu (encadré $\mathrm{n}^{\circ} 2$ ). A cet effet, les opportunités récentes offertes par les technologies mobiles et le mobile money apparaissent pour certains comme de nouveaux dispositifs permettant de constituer une "épargne légère » (entretien n ${ }^{\circ} 26$ ). A côté de cet effort d'épargne, les entretiens révèlent aussi un effort partagé par l'ensemble de ces catégories intermédiaires de revenus pour l'éducation des enfants qui apparait comme une de leur préoccupation majeure. Les données quantitatives sont assez éloquentes à cet égard. Les ménages de la classe moyenne consacrent environ $2 \%$ de leur revenu annuel aux dépenses d'éducation, soit un montant annuel moyen de 45000 FCFA ce qui représente le double du montant consacré par la catégorie des pauvres au dépenses d'éducation et deux tiers du montant dépensé par les $5 \%$ les plus riches ${ }^{21}$. Dans l'ensemble les dépenses de consommation des ménages de la classe moyenne, tout en restant nettement inférieures à celle des plus riches, se distinguent assez nettement de celle des plus pauvres dans les domaines des dépenses d'habillement, de transport et de communication. Les dépenses de transport représentent en moyenne 10\% de leur revenu. Le poids important de ce poste de dépense est à mettre en relation avec des problématiques liées à l'accès au logement qui pour se faire à des prix raisonnable conduit souvent ces ménages à s'éloigner de leur lieu de travail. Il est à noter que la classe moyenne se différencie peu avec les plus pauvres sur le montant des dépenses consacrées à la santé, traduisant par là une relative vulnérabilité. Les ménages de la classe moyenne dépensent en moyenne 9,1\% de leur revenu en transferts. Les effets de ces transferts sont probablement ambigus entre maintient dans les réseaux de solidarités et de redistribution familiaux et claniques et compression des autres postes budgétaires... Le positionnement des individus la classe moyenne dans les réseaux de solidarité est potentiellement asymétrique du fait de leur capacité à venir en aide aux membres les plus pauvres mais de leur

21 Calculs non présentés. 
difficulté à accéder eux même à du soutien financier en cas de besoin (Kroeker, 2016). Au-delà de l'aspect contraignant qui se dégage des propos des enquêtés, renforcer les liens avec le lieu d'origine, rechercher la protection et la reconnaissance sociale des membres du groupe d'appartenance sont les logiques qui sous-tendent l'action d'envoi d'argent au sein de ces classes moyennes. Mais on observe dans le même temps des stratégies de contournement assez bien exprimés par le cas $n^{\circ} 26$ (employé dans une entreprise privée) dont le choix de résidence à Yopougon s'explique notamment par sa volonté de s'éloigner géographiquement de sa famille, afin de pouvoir développer son autonomie (étant dernier de famille, il était sujet à être « corvéable » à merci).

\title{
Encadré $n^{\circ} 2$ - Extraits des entretiens au regard du comportement d'épargne
}

\begin{abstract}
"J'ai souscrit à un compte d'épargne mouvement, donc il y a déjà une ponction qui est faites à la base. Mais en plus de cela, moi-même j'enlève quelque chose dessus pour mettre de côté parce qu'on ne sait jamais, au cas où je suis confronté à une difficulté particulière voilà. Quand Je n'ai pas de soucis particuliers, je peux mettre entre 20\% et 30\% de mon salaire à l'épargne ». Extrait de l'entretien avec l'enquêté 3, 46 ans, chef de service à la poste, la taille du ménage est de 5 personnes.

"J'ai au moins trois comptes chaque semaine je peux mettre dans chaque compte 100000, 200000, ça dépend... Je suis dans au moins 3 tontines, il y a un on fait 10000 par jour, ily a un on fait 5000 par jour et puis il y a un autre aussi on fait 10000 par jour». Extrait de l'entretien avec l'enquêté 14, 30 ans, commerçant de vêtement prêt à porter, la taille du ménage est de 3 personnes.
\end{abstract}

Les données qualitatives permettent également de préciser quelques modalités pratiques des comportements et lieux de consommation. De manière générale il ressort que les ménages, pour l'essentiel, se rendent dans les marchés de quartiers et quelques supermarchés pour leurs achats (à des fréquences qui varient en fonction des besoins et des lieux d'achat). Les ménages, plus précisément la conjointe ou le personnel domestique se rendent au moins une fois par semaine dans les marchés de quartier pour l'achat des produits servant aux repas quotidiens (viande, poisson, légumes, céréales...). Tandis que les courses dans les supermarchés sont généralement programmées pour les fins de mois et concernent les produits d'hygiène et de soin. A ce niveau, les courses sont souvent réalisées par l'homme, chef de ménage. 
Encadré $n^{\circ} 3$ - Extraits des entretiens au regard des dépenses de transfert

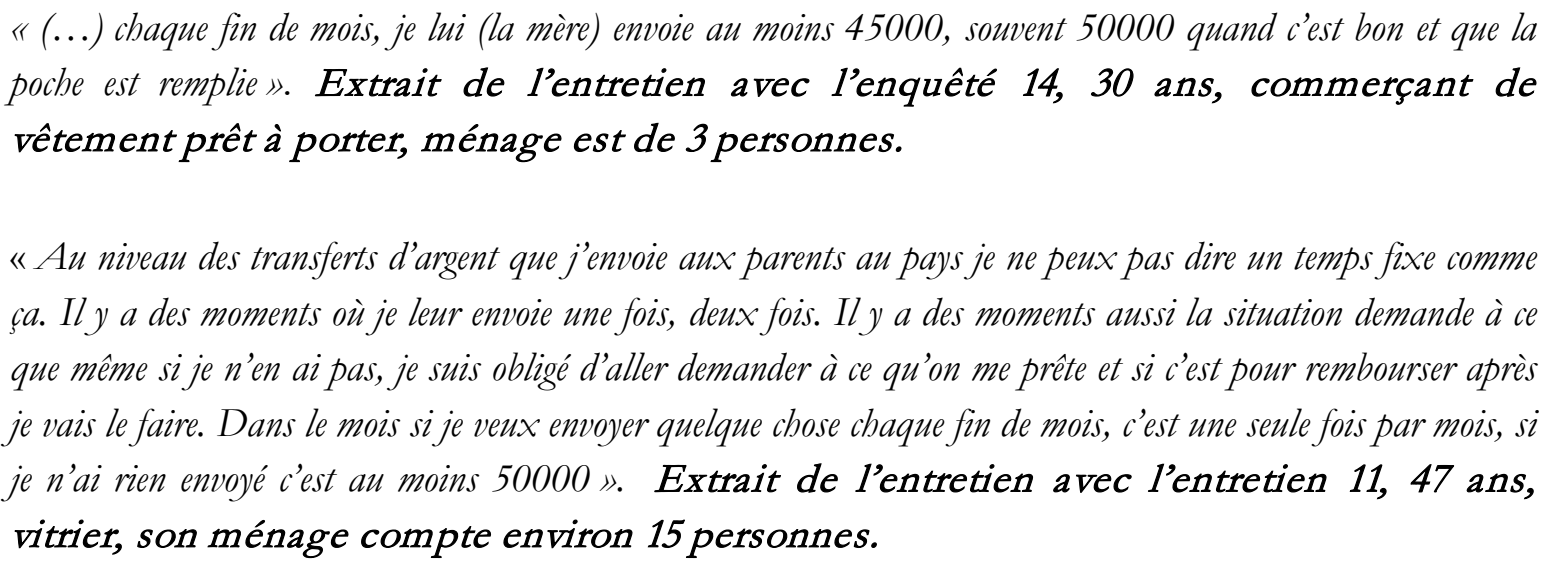

"Au niveau des transferts d'argent que j'envoie aux parents au pays je ne peux pas dire un temps fixe comme ça. Il y a des moments où je leur envoie une fois, deux fois. Il y a des moments aussi la situation demande à ce que même si je n'en ai pas, je suis obligé d'aller demander à ce qu'on me prête et si c'est pour rembourser après je vais le faire. Dans le mois si je veux envoyer quelque chose chaque fin de mois, c'est une seule fois par mois, si je n'ai rien envoyé c'est au moins 50000 ». Extrait de l'entretien avec l'entretien 11, 47 ans, vitrier, son ménage compte environ 15 personnes.

Enfin, sur le plan des aspirations on note des aspirations communes à assurer l'avenir de leurs enfants à travers l'éducation. Le regard de ces individus de la classe moyenne sur la politique est assez amer. De manière transversale on note une dénonciation commune de l'accroissement des inégalités et de la corruption des élites caractérisant une "Côte d'Ivoire à deux vitesses ». L'écart entre les discours sur la croissance à 2 chiffres et les conditions de la majorité qui ne cessent de se détériorer est souvent dénoncé : "Le sac de sucre est à 800Fcfa, mais on a une croissance à 2 chiffres! on mange 2 repas par jour, mais on a une croissance à 2 chiffres! » (entretien $\left.\mathrm{n}^{\circ} 26\right)$.

Ces différents éléments de caractérisation tendent à montrer qu'il existe quelques marqueurs forts d'appartenance à la classe moyenne ivoirienne. Pour autant, au regard de l'étendue des situations possibles en termes de revenus ( $\mathrm{cf}$. figure $\mathrm{n}^{\circ} 3$ ) cette classe moyenne est probablement hétérogène en termes de caractéristiques socioéconomiques. Il s'agit alors d'étudier la cohérence de ce groupe de $26,4 \%$ de la population pour passer d'une catégorie limitée de " gens du milieu » à une catégorie éclatée « des gens des milieux sociaux différents ».

\subsection{L'hétérogénéité de la classe moyenne ivoirienne : des gens des milieux sociaux différents.}

A ce stade de l'analyse, l'objectif est de mettre en évidence la potentielle hétérogénéité de la classe moyenne ivoirienne. La démarche consiste alors à mobiliser les informations plus qualitatives sur l'emploi et l'éducation afin d'identifier les différentes composantes de l'ensemble " classe moyenne » délimité lors de l'étape précédente.

\subsubsection{La stratégie d'identification des groupes composant la classe moyenne ivoirienne}

Afin d'explorer l'hétérogénéité de cette classe moyenne " de revenu » d'un point de vue multidimensionnel, nous mettons en œuvre une procédure de classification mixte sur plusieurs variables décrivant les statuts occupationnels et éducatifs des ménages de l'échantillon considéré. Plus précisément, après analyse des questionnaires d'enquêtes, nous avons sélectionné et recodé cinq variables liées à l'éducation et à l'emploi pour mettre en œuvre la classification : (i) le niveau 
d'éducation le plus élevé atteint par le chef de ménage, (ii) la catégorie socio-professionnelle du chef de ménage, (iii) le statut dans l'emploi du chef de ménage, (iv) le type d'employeur ou secteur institutionnel du chef de ménage et (v) la pluriactivité du chef de ménage.

Sur la base de ces cinq variables, la procédure retenue effectue une classification d'un grand ensemble d'individus caractérisés par leurs premières coordonnées factorielles créées par une procédure initiale d'analyse factorielle (ici une analyse des correspondances multiples menée sur les cinq variables). Une première classification est obtenue par croisement de plusieurs partitions de base construites autour de centres mobiles, puis les classes stables ainsi formées sont agrégées par une méthode de classification hiérarchique. Le critère d'agrégation ici retenu est le critère de Ward. La partition que nous retiendrons (le nombre de groupes retenus au sein de la classe moyenne de revenus) résulte de l'analyse de l'histogramme des indices de niveau d'agrégation des nœuds de la hiérarchie et de l'analyse du dendrogramme (schéma synthétique de l'arbre d'agrégation). Sur cette base, nous proposerons une classification en groupes homogènes et clairement distincts les uns des autres. Afin de les caractériser au mieux, nous comparons enfin d'un groupe à l'autre les distributions des différentes variables de classification citées plus haut (tableau $\mathrm{n}^{\circ}$ ) et affinerons l'analyse en comparant les distributions ou les moyennes d'un ensemble d'autres variables additionnelles, dites variables de caractérisation (tableau $n^{\circ} 6$ ). Les entretiens qualitatifs réalisés auprès d'un échantillon de ménages représentant les différents groupes identifiés nous aideront également à compléter la caractérisation de ces groupes (encadré $\mathrm{n}^{\circ} 1$ et tableau $\mathrm{n}^{\circ} \mathrm{A} 1$ en annexe).

\section{Encadré $n^{\circ} 4-$ Grille de lecture des tableaux n ${ }^{\circ} 5$ et 6}




\subsubsection{Une typologie de la classe moyenne ivoirienne: description des cinq composantes de la classe moyenne}

Les résultats de la classification nous conduisent à identifier cinq groupes distincts au sein de la classe moyenne ivoirienne en 2015.

Le premier groupe identifié est celui de la classe moyenne des agriculteurs $(25 \%$ de l'ensemble de la classe moyenne). Cette partie de la classe moyenne ivoirienne est constituée d'agriculteurs (ainsi que des pêcheurs et des exploitants forestiers) indépendants informels. Ceuxci sont assez fortement représentés dans le Sud-Ouest du pays. Ils exercent évidemment très majoritairement en milieu rural et sont, bien plus que les autres, propriétaires de leurs habitations. Près de 65\% d'entre eux n'ont pas atteint le niveau de scolarité primaire. Les chefs de ces ménages, qui à $91 \%$ sont des hommes, sont en moyenne relativement âgés et se distinguent par la relative faiblesse de la part de leurs dépenses consacrées à l'éducation et aux communications. Les entretiens qualitatifs réalisés auprès des ménages de cette catégorie ont à ce titre révélé leurs difficultés d'accès à l'internet et aux équipements en TIC. La faiblesse de leurs dépenses en éducation, résultant de la faiblesse de leur revenu, contraste évidemment avec l'importance que les interviewés accordent à cet enjeu (cf. encadré $n^{\circ} 5$ ). Le cas de Mr. T. (entretien $n^{\circ} 26$ ), agriculteur de 47 ans, est assez éloquent à ce sujet. L'éducation de ses enfants est une priorité pour Mr. T., qui souhaite que ceux-ci le dépassent et réussissent leurs vies. Il investit massivement dans ce secteur, aidé par des dons extérieurs. Il a choisi une école semi-privée pour son fils ainé (18ans), qui est en terminale technique, avec un coût de 95.000 FCFA / an ; pour son autre fille ainée (jumelle de 18ans), qui a vécu un échec scolaire sans obtenir son BEPC, il a choisi de l'inscrire dans une école privée (200.000 FCFA /an), où elle était acceptée, bien qu'il dise que ce choix soit un pari «fou ». Sa 3ème fille est en 5ème, dans une école publique (35.000 FCFA par an), et sa dernière dans une école primaire publique. Ces coûts ne comportent pas les frais de fourniture scolaire importants. Ses 3 ainés vivent dans des familles en ville (à Angré), aidés par des amis et de la famille. Mr. T. estime que l'école en Côte d'Ivoire est extrêmement coûteuse, mais choisit de sacrifier son niveau de vie pour offrir une chance à ses enfants. C'est ainsi qu'ils se rationnent, au niveau de leur alimentation. 
Encadré $n^{\circ} 5$ - La question de l'éducation pour la classe moyenne des agriculteurs

"Très (optimiste), parce que la manière dont je me suis donné des objectifs pour mon champ, c'est la même chose je veux que cela rejaillit sur mes enfants. Parce qu'objectivement si j'ai créé ces champs, c'est pour avoir des revenus. Et ces revenus ne seront pas dilapidés. Je vais intéresser ça à les mettre dans de bonnes conditions, dans de bonnes écoles pour qu'ils apprennent dans de très bonnes conditions également pour leur réussite. 》

Extrait de l'entretien avec l'enquêté 1 de la classe moyenne des agriculteurs informels, 44 ans, ménage comprenant au total 5 personnes.

"Ceux qui pourront avoir les diplômes et n'ont pas pu avoir quelque chose, ily a l'auto-emploi qui est là ; c'est pourquoi je me suis mis en brousse pour pouvoir me faire, je leur dis toujours. Si demain même vous avez eu des diplômes et vous n'avez, pas eu quelque chose à faire, peut-être que les champs que je suis en train de faire, vont vous permettre d'avoir vos revenus et mener une activité; mais dans un premier temps, avant de dire que je ne trouve pas du travail, il faut avoir ton diplôme»

Extrait de l'entretien avec l'enquêté 7, 47 ans, de la catégorie de la classe moyenne de l'agriculture informelle. La taille du ménage est de 12 personnes.

Les interviewés expriment dans l'ensemble une relative satisfaction à l'égard de leur niveau de vie, ne se sentant «ni riches ni pauvres », mais affichent leurs inquiétudes au regard de la vulnérabilité de leur situation face aux différents chocs (fluctuation des prix, instabilité foncière, santé...). Leurs histoires soulignent la force avec laquelle certains événements de la vie impactent de manière durable leur existence (perte d'un membre de la famille, succession difficile, champs brûlé, climat...), et les difficultés à pouvoir s'en relever, pour améliorer leurs conditions d'existence. La gestion de ces risques passe souvent par une stratégie de diversification des cultures et des activités. Elle les conduit également à ajuster leurs comportements de consommation et à «vivre selon la courbe » selon l'expression de Mr. T. (entretien $n^{\circ} 26$ ). Leurs préoccupations touchent ainsi principalement à leurs conditions matérielles de vie et à l'avenir de leurs enfants. Ils sont peu engagés dans des formes d'actions ou de revendications collectives et leur degré d'implication dans la vie sociale et politique est quasiment nul. Pour autant, ils témoignent d'un sentiment de déclassement et de dévalorisation de leur situation. Mr. T. (entretien $\left.n^{\circ} 26\right)$ souligne que si au temps d'Houphouët, les agriculteurs étaient valorisés (avec notamment une couverture sociale maladie), il ressent aujourd'hui une forte indifférence, un abandon, voire un mépris des pouvoirs publics à leur égard. 
Tableau nº - Distributions et moyennes comparées des variables de caractérisation selon la classe* (Côte d'Ivoire 2015)

\begin{tabular}{|c|c|c|c|c|c|c|}
\hline \multicolumn{7}{|l|}{ SECTEUR D'ACTIVITE } \\
\hline \multicolumn{7}{|l|}{ Effectif } \\
\hline \multirow{2}{*}{\multicolumn{7}{|c|}{\begin{tabular}{l|l|l|l|} 
Classe 3 & Classe 4 & Classe 5 & Ensemble
\end{tabular}}} \\
\hline & & & & & & \\
\hline & 796 & 285 & 18 & 56 & 85 & 1240 \\
\hline \multirow{3}{*}{ Agriculture, pêche, forêt } & 64,2 & 23,0 & 1,5 & 4,5 & 6,9 & 100,0 \\
\hline & 92,8 & 21,4 & 14,0 & 9,5 & 55,2 & 40,5 \\
\hline & 16 & 245 & 23 & 58 & 12 & 354 \\
\hline \multirow[t]{3}{*}{ Industrie manufacturière, extractive } & 4,5 & 69,2 & 6,5 & 16,4 & 3,4 & 100,0 \\
\hline & 1,9 & 18,4 & 17,8 & 9,9 & 7,8 & 11,6 \\
\hline & 0 & 79 & 6 & 15 & 5 & 105 \\
\hline \multirow[t]{3}{*}{ Construction } & 0,0 & 75,2 & 5,7 & 14,3 & 4,8 & 100,0 \\
\hline & 0,0 & 5,9 & 4,7 & 2,6 & 3,3 & 3,4 \\
\hline & 29 & 412 & 22 & 42 & 19 & 524 \\
\hline \multirow[t]{3}{*}{ Commerce gros et détail } & 5,5 & 78,6 & 4,2 & 8,0 & 3,6 & 100,0 \\
\hline & 3,4 & 30,9 & 17,1 & 7,2 & 12,3 & 17,1 \\
\hline & 6 & 51 & 3 & 9 & 0 & 69 \\
\hline \multirow{3}{*}{ Hôtellerie et restauration } & 8,7 & 73,9 & 4,4 & 13,0 & 0,0 & 100,0 \\
\hline & 0,7 & 3,8 & 2,3 & 1,5 & 0,0 & 2,3 \\
\hline & 0 & 94 & 12 & 54 & 2 & 162 \\
\hline \multirow[t]{3}{*}{ Transports } & 0,0 & 58,0 & 7,4 & 33,3 & 1,2 & 100,0 \\
\hline & 0,0 & 7,0 & 9,3 & 9,2 & 1,3 & 5,3 \\
\hline & 1 & 57 & 23 & 43 & 6 & 130 \\
\hline \multirow[t]{3}{*}{ Immobilier, act. Financières } & 0,8 & 43,9 & 17,7 & 33,1 & 4,6 & 100,0 \\
\hline & 0,1 & 4,3 & 17,8 & 7,3 & 3,9 & 4,2 \\
\hline & 0 & 9 & 4 & 65 & 1 & 79 \\
\hline \multirow[t]{3}{*}{ Administration publique } & 0,0 & 11,4 & 5,1 & 82,3 & 1,3 & 100,0 \\
\hline & 0,0 & 0,7 & 3,1 & 11,1 & 0,7 & 2,6 \\
\hline & 2 & 33 & 8 & 202 & 17 & 262 \\
\hline \multirow[t]{3}{*}{ Education } & 0,8 & 12,6 & 3,1 & 77,1 & 6,5 & 100,0 \\
\hline & 0,2 & 2,5 & 6,2 & 34,4 & 11,0 & 8,6 \\
\hline & 1 & 15 & 3 & 27 & 3 & 49 \\
\hline \multirow[t]{3}{*}{ Santé, action sociale } & 2,0 & 30,6 & 6,1 & 55,1 & 6,1 & 100,0 \\
\hline & 0,1 & 1,1 & 2,3 & 4,6 & 2,0 & 1,6 \\
\hline & 7 & 55 & 7 & 16 & 4 & 89 \\
\hline \multirow[t]{3}{*}{ Autres services } & 7,9 & 61,8 & 7,9 & 18,0 & 4,5 & 100,0 \\
\hline & \begin{tabular}{l|l}
0,8 \\
\end{tabular} & 4,1 & 5,4 & 2,7 & 2,6 & 2,9 \\
\hline & 858 & 1335 & 129 & 587 & 154 & 3063 \\
\hline \multirow[t]{2}{*}{ Ensemble } & 28,0 & 43,6 & 4,2 & 19,2 & 5,0 & 100,0 \\
\hline & 100,0 & 100,0 & 100,0 & 100,0 & 100,0 & 100,0 \\
\hline
\end{tabular}

\begin{tabular}{|c|c|c|c|c|c|c|}
\hline \multicolumn{7}{|l|}{ REGION } \\
\hline \begin{tabular}{|l|} 
Effectif \\
$\%$ en ligne \\
$\%$ en colonne
\end{tabular} & Classe 1 & Classe 2 & Classe 3 & Classe 4 & Classe 5 & Ensemble \\
\hline & 15 & 269 & 48 & 152 & 70 & 554 \\
\hline Ville d'Abidjan & 2,7 & 48,6 & 8,7 & 27,4 & 12,6 & 100,0 \\
\hline & 1,8 & 20,1 & 37,2 & 25,9 & 14,2 & 16,3 \\
\hline & 179 & 126 & 13 & 31 & 28 & 377 \\
\hline \begin{tabular}{|l|} 
Sud-Ouest \\
\end{tabular} & 47,5 & 33,4 & 3,5 & 8,2 & 7,4 & 100,0 \\
\hline & 20,9 & 9,4 & 10,1 & 5,3 & 5,7 & 11,1 \\
\hline & 128 & 234 & 27 & 97 & 88 & 574 \\
\hline Sud & 22,3 & 40,8 & 4,7 & 16,9 & 15,3 & 100,0 \\
\hline & 14,9 & 17,5 & 20,9 & 16,5 & 17,9 & 16,9 \\
\hline & 93 & 100 & 8 & 30 & 30 & 261 \\
\hline Ouest & 35,6 & 38,3 & 3,1 & 11,5 & 11,5 & 100,0 \\
\hline & 10,8 & 7,5 & 6,2 & 5,1 & 6,1 & 7,7 \\
\hline & 97 & 72 & 3 & 42 & 41 & 255 \\
\hline Nord-Ouest & 38,0 & 28,2 & 1,2 & 16,5 & 16,1 & 100,0 \\
\hline & 11,3 & 5,4 & 2,3 & 7,2 & 8,3 & 7,5 \\
\hline & 48 & 35 & 3 & 21 & 23 & 130 \\
\hline \begin{tabular}{|l|} 
Nord-Est \\
\end{tabular} & 36,9 & 26,9 & 2,3 & 16,2 & 17,7 & 100,0 \\
\hline & 5,6 & 2,6 & 2,3 & 3,6 & 4,7 & 3,8 \\
\hline & 61 & 71 & 5 & 35 & 21 & 193 \\
\hline Nord & 31,6 & 36,8 & 2,6 & 18,1 & 10,9 & 100,0 \\
\hline & 7,1 & 5,3 & 3,9 & 6,0 & 4,3 & 5,7 \\
\hline & 123 & 173 & 3 & 41 & 63 & 403 \\
\hline \begin{tabular}{|l|} 
Centre-Ouest \\
\end{tabular} & 30,5 & 42,9 & 0,7 & 10,2 & 15,6 & 100,0 \\
\hline & 14,3 & 13,0 & 2,3 & 7,0 & 12,8 & 11,8 \\
\hline & 16,0 & 78,0 & 6,0 & 41,0 & 31,0 & 172,0 \\
\hline \begin{tabular}{|l|} 
Centre-Nord \\
\end{tabular} & 9,3 & 45,4 & 3,5 & 23,8 & 18,0 & 100,0 \\
\hline & 1,9 & 5,8 & 4,7 & 7,0 & 6,3 & 5,1 \\
\hline & 20,0 & 60,0 & 4,0 & 15,0 & 12,0 & 111,0 \\
\hline \begin{tabular}{|l|} 
Centre-Est \\
\end{tabular} & 18,0 & 54,1 & 3,6 & 13,5 & 10,8 & 100,0 \\
\hline & 2,3 & 4,5 & 3,1 & 2,6 & 2,4 & 3,3 \\
\hline & 78 & 118 & 9 & 82 & 86 & 373 \\
\hline Centre & 20,9 & 31,6 & 2,4 & 22,0 & 23,1 & 100,0 \\
\hline & 9,1 & 8,8 & 7,0 & 14,0 & 17,4 & 11,0 \\
\hline & 858 & 1336 & 129 & \begin{tabular}{|l|}
587 \\
\end{tabular} & \begin{tabular}{l|l}
493 \\
\end{tabular} & 3403 \\
\hline Ensemble & 25,2 & 39,3 & 3,8 & \begin{tabular}{|l|}
17,3 \\
\end{tabular} & 14,5 & 100,0 \\
\hline & 100,0 & 100,0 & 100,0 & 100,0 & 100,0 & 100,0 \\
\hline
\end{tabular}

(*) Les cases grisées dans le tableau identifient les modalités qui, d'un point de vue statistique, sont significativement surreprésentées dans la classe considérée par rapport au reste de la population.

Source : données ENV (2015) 
Tableau n ${ }^{\circ} 6$ (suite) - Distributions et moyennes comparées des variables de caractérisation selon la classe* (Côte d'Ivoire 2015)

\begin{tabular}{|l|r|r|r|r|r|r|}
\hline GENRE & \multicolumn{1}{|c|}{ Classe 1 } & Classe 2 & Classe 3 & Classe 4 & Classe 5 & Ensemble \\
$\begin{array}{l}\% \text { en ligne } \\
\text { \% en colonne }\end{array}$ & 783 & 1101 & 121 & 506 & 351 & 2862 \\
\hline & 27,4 & 38,5 & 4,2 & 17,7 & 12,3 & 100,0 \\
\hline Homme & 91,3 & 82,4 & 93,8 & 86,2 & 71,2 & 84,1 \\
\hline & 75 & 235 & 8 & 81 & 142 & 541 \\
\hline & 13,9 & 43,4 & 1,5 & 15,0 & 26,3 & 100,0 \\
\hline Femme & 8,7 & 17,6 & 6,2 & 13,8 & 28,8 & 15,9 \\
\hline & 858 & 1336 & 129 & 587 & 493 & 3403 \\
\hline Ensemble & 25,2 & 39,3 & 3,8 & 17,3 & 14,5 & 100,0 \\
\hline & 100,0 & 100,0 & 100,0 & 100,0 & 100,0 & 100,0 \\
\hline
\end{tabular}

\section{NATI ONALITE}

\begin{tabular}{|l|r|r|r|r|r|r|}
\hline $\begin{array}{l}\text { NATI ONALITE } \\
\text { \% en ligne } \\
\text { \% en colonne }\end{array}$ & Classe 1 & Classe 2 & Classe 3 & Classe 4 & Classe 5 & Ensemble \\
\hline & 619 & 896 & 110 & 524 & 410 & 2559 \\
\hline Ivoirienne & 24,2 & 35,0 & 4,3 & 20,5 & 16,0 & 100,0 \\
\hline & 72,1 & 67,1 & 85,3 & 89,3 & 83,2 & 75,2 \\
\hline & 239 & 440 & 19 & 63 & 83 & 844 \\
\hline Non ivoirienne & 28,3 & 52,1 & 2,3 & 7,5 & 9,8 & 100,0 \\
\hline & 27,9 & 32,9 & 14,7 & 10,7 & 16,8 & 24,8 \\
\hline & 858 & 1336 & 129 & 587 & 493 & 3403 \\
\hline Ensemble & 25,2 & 39,3 & 3,8 & 17,3 & 14,5 & 100,0 \\
\hline & 100,0 & 100,0 & 100,0 & 100,0 & 100,0 & 100,0 \\
\hline
\end{tabular}

\section{LIEU DE RESI DENCE}

\section{Effectif}

$\%$ en ligne

$\%$ en ligne
$\%$ en colonne

(1)

Abidjan

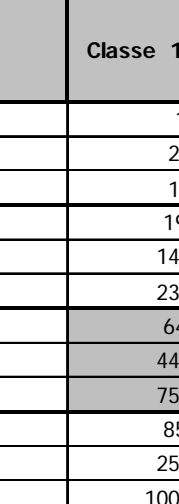

\begin{tabular}{|r|r|r|r|r|r|}
\hline $\mathbf{1}$ & Classe 2 & Classe 3 & Classe 4 & Classe 5 & Ensemble \\
\hline 15 & 269 & 48 & 152 & 70 & 554 \\
\hline 2,7 & 48,6 & 8,7 & 27,4 & 12,6 & 100,0 \\
\hline 1,8 & 20,1 & 37,2 & 25,9 & 14,2 & 16,3 \\
\hline 199 & 604 & 56 & 314 & 235 & 1408 \\
\hline 14,1 & 42,9 & 4,0 & 22,3 & 16,7 & 100,0 \\
\hline 23,2 & 45,2 & 43,4 & 53,5 & 47,7 & 41,4 \\
\hline 644 & 463 & 25 & 121 & 188 & 1441 \\
\hline 44,7 & 32,1 & 1,7 & 8,4 & 13,1 & 100,0 \\
\hline 75,1 & 34,7 & 19,4 & 20,6 & 38,1 & 42,3 \\
\hline 858 & 1336 & 129 & 587 & 493 & 3403 \\
\hline 25,2 & 39,3 & 3,8 & 17,3 & 14,5 & 100,0 \\
\hline 100,0 & 100,0 & 100,0 & 100,0 & 100,0 & 100,0 \\
\hline
\end{tabular}

\begin{tabular}{|l|r|r|r|r|r|r|}
\hline $\begin{array}{l}\text { MODALITE D'ACCES A L'EMPLOI } \\
\text { \% en ligne } \\
\text { \% en colonne }\end{array}$ & Classe 1 & Classe 2 & Classe 3 & Classe 4 & Classe 5 & Ensemble \\
\hline & 365 & 614 & 56 & 377 & 63 & 1475 \\
\hline Autres modalités d'accès & 24,8 & 41,6 & 3,8 & 25,6 & 4,3 & 100,0 \\
\hline & 42,5 & 46,0 & 43,4 & 64,2 & 46,7 & 48,5 \\
\hline & 493 & 721 & 73 & 210 & 72 & 1569 \\
\hline Relation personnelle & 31,4 & 46,0 & 4,7 & 13,4 & 4,6 & 100,0 \\
\hline & 57,5 & 54,0 & 56,6 & 35,8 & 53,3 & 51,5 \\
\hline & 858 & 1335 & 129 & 587 & 135 & 3044 \\
\hline Ensemble & 28,2 & 43,9 & 4,2 & 19,3 & 4,4 & 100,0 \\
\hline & 100,0 & 100,0 & 100,0 & 100,0 & 100,0 & 100,0 \\
\hline
\end{tabular}

100,0
MOYENNES VARI ABLES QUANTI TATI VES

\begin{tabular}{|l|r|r|r|r|r|r|}
\hline $\begin{array}{l}\text { Moyenne } \\
\text { Ecart-type }\end{array}$ & Classe 1 & Classe 2 & Classe 3 & Classe 4 & Classe 5 & Ensemble \\
\hline & 2,9 & 2,5 & 3,6 & 3,9 & 2,8 & 2,9 \\
\hline Taille du ménage & 2,0 & 1,8 & 2,3 & 2,4 & 2,1 & 2,1 \\
\hline Possédez-vous une habitation & 0,77 & 0,34 & 0,19 & 0,28 & 0,52 & 0,46 \\
\hline & 0,42 & 0,47 & 0,39 & 0,45 & 0,50 & 0,50 \\
\hline Revenu du chef / Rev. MEN & 0,91 & 0,89 & 0,88 & 0,88 & 0,81 & 0,88 \\
\hline Revenu mensuel MEN & 0,17 & 0,21 & 0,19 & 0,20 & 0,31 & 0,22 \\
\hline & 149623 & 132035 & 217197 & 235827 & 148874 & 160041 \\
\hline Revenu mensuel par habitant & 118926 & 107437 & 173646 & 173740 & 142591 & 137241 \\
\hline Age du chef de ménage & 54778 & 57162 & 62710 & 64699 & 52503 & 57396 \\
\hline & 24924 & 24731 & 26105 & 28485 & 24708 & 25801 \\
\hline Utilisation internet & 43,1 & 36,2 & 39,9 & 39,6 & 45,3 & 40,0 \\
\hline & 13,7 & 11,9 & 11,1 & 10,3 & 19,3 & 13,8 \\
\hline Part des dépenses éducation & 0,01 & 0,07 & 0,36 & 0,29 & 0,08 & 0,10 \\
\hline & 0,11 & 0,25 & 0,48 & 0,45 & 0,27 & 0,31 \\
\hline Part des dépenses communicat & 0,004 & 0,013 & 0,034 & 0,032 & 0,030 & 0,017 \\
\hline & 0,019 & 0,042 & 0,062 & 0,066 & 0,087 & 0,054 \\
\hline Part des dépenses transport & 0,045 & 0,066 & 0,078 & 0,071 & 0,065 & 0,062 \\
\hline & 0,057 & 0,088 & 0,059 & 0,073 & 0,083 & 0,078 \\
\hline Part des dépenses habillement & 0,104 & 0,105 & 0,098 & 0,118 & 0,090 & 0,104 \\
\hline & 0,187 & 0,242 & 0,152 & 0,276 & 0,165 & 0,223 \\
\hline Part des dépenses santé & 0,053 & 0,055 & 0,050 & 0,050 & 0,046 & 0,052 \\
\hline & 0,046 & 0,049 & 0,043 & 0,045 & 0,054 & 0,048 \\
\hline Part des dépenses de transferts & 0,023 & 0,017 & 0,035 & 0,021 & 0,031 & 0,022 \\
\hline & 0,105 & 0,049 & 0,103 & 0,054 & 0,100 & 0,078 \\
\hline & 0,108 & 0,088 & 0,093 & 0,093 & 0,066 & 0,091 \\
\hline
\end{tabular}

(*) Les cases grisées dans le tableau identifient les modalités qui, d'un point de vue statistique, sont significativement surreprésentées dans la classe considérée par rapport au reste de la population.

Source : données ENV (2015) 
Le second groupe est celui de la classe moyenne des travailleurs de l'économie informelle, il représente $39 \%$ de l'ensemble de la classe moyenne. Cet ensemble de ménages est presque intégralement constitué d'employés et travailleurs des services et d'ouvriers qui exercent dans l'économie informelle. Les chefs de ces ménages sont pour l'essentiel indépendants ou employés de l'informel. On les trouve beaucoup dans l'industrie manufacturière et le commerce de gros et de détail, et pour 65\% d'entre eux en milieu urbain (dont 20\% à Abidjan). Parmi l'ensemble des chefs de ménages de cette catégorie, près de $18 \%$ sont des femmes. De manière générale, les chefs de ménage de cette catégorie se caractérisent par de nombreux éléments de vulnérabilité. En terme de capital humain, 75\% d'entre eux disposent d'un niveau d'éducation primaire ou inférieur. Les chefs de ménages de nationalité non-ivoirienne sont surreprésentés dans cette catégorie. Ils sont relativement jeunes comparativement à l'ensemble de la classe moyenne et leurs ménages sont en moyenne plus petits. Ils disposent du niveau moyen de revenu par ménage le plus faible sur l'ensemble de la classe moyenne et la part de leurs dépenses en éducation et santé est particulièrement faible. Les entretiens qualitatifs ont par ailleurs mis en évidence les conditions de vie difficiles de cette catégorie. Mme A. (entretien n²8), âgée de 38 ans et gérante d'un salon de coiffure à Cocody, représente bien cette situation, elle s'identifie comme étant moins pauvre que les pauvres, mais très éloignée des riches, avec des conditions de vie quotidiennes globalement difficiles. Ses besoins en termes de logement, de santé et d'éducation ne sont pas pleinement comblés (logement petit et saleté du quartier ; arbitrage budgétaire dans les soins de santé ; retour de sa fille dans une école publique surpeuplée). Elle effectue donc des arbitrages sur sa consommation directe (diminution de sa consommation de riz, par exemple, passant de $25 \mathrm{~kg}$ par mois à $15 \mathrm{~kg} / \mathrm{mois}$ ), tout en veillant à développer une petite épargne, pour investir dans un projet futur, où elle serait à son compte. Elle développe des stratégies individuelles, ne pouvant compter que sur elle-même, sans aide de proches (femme seule avec enfant, sans aide du père de l'enfant ou d'autres personnes), ni de l'Etat. Elle souhaite conserver un certain optimisme vis-à-vis de l'avenir, nourrissant des projets futurs, tout en faisant face à des difficultés quotidiennes au niveau de ses conditions matérielles. Issue d'un milieu économique rural et difficile (elle a dû d'abord partir de chez sa mère pour être élevée par son oncle et sa tante, puis arrêter l'école en $6^{\text {ème }}$ pour travailler aux champs), elle pense vivre une petite ascension sociale, bien que celle-ci reste limitée et en-deçà de ses ambitions. Le cas de Mme A., comme les autres d'ailleurs, témoigne ainsi d'une tendance à la reproduction des positions sociales. Ces indépendants ont, pour la majorité, hérité ou reproduit les milieux d'affaires de leurs parents ou sont issus de parents agriculteurs informels, mais jouissent de revenus quelque peu supérieures à ces derniers. Leur trajectoire passe souvent par des phases d'apprentissage et de formation sur le tas. Elle est non linéaire et repose sur des stratégies d'accumulations économique et sociale leur permettant petit à petit de constituer leur propre business. L'épargne passe le plus souvent par des canaux informels ou des structures plus adaptées à leurs besoins (microfinance, mobile money). On remarque que parmi les personnes interrogées, les choix de scolarisation des enfants se portent principalement vers les établissements privés ce qui représente un gros investissement pour ces ménages. Malgré des conditions difficiles qui les éloignent quelque peu de l'image parfois idéalisé des «success stories» d'un entreprenariat dynamique et innovant (UCTUNILEVER, 2017), les interviewés témoignent d'aspirations à devenir de véritables 
entrepreneurs puisqu'ils ont souvent l'ambition de développer et d'intensifier leur activité, même de devenir dirigeants de plus grandes entreprises.

Le troisième groupe est celui de la classe moyenne intermédiaire du secteur privé formel ( $4 \%$ du total de la classe moyenne). Ce groupe rassemble des salariés et employés du secteur privé formel qui exercent en tant que professions intermédiaires, employés ou ouvriers. On les retrouve dans presque toutes les activités, mais ils sont surreprésentés dans les activités immobilières et financières. Par ailleurs, près de $40 \%$ d'entre eux exercent à Abidjan contre seulement $16 \%$ de l'ensemble des ménages de la classe moyenne. Les chefs de ces ménages de grande taille sont bien plus rarement propriétaires de leurs logements que les autres. Leurs revenus moyens sont relativement élevés. Ils utilisent bien plus internet que les autres groupes et se distinguent nettement des autres par la part de leurs revenus allouée à l'éducation, aux communications et à la santé. Les entretiens qualitatifs menés auprès des chefs de ménage de cette catégorie soulignent une forme de transmission intergénérationnelle du statut de classe moyenne puisque leurs parents ont bien souvent été des fonctionnaires de l'administration publique ivoirienne (douanier, gendarme, techniciens de travaux publics) dans les années 19601970 (encadré nº).

\title{
Encadré $n^{\circ} 6$ - Extrait de l'entretien $n^{\circ} 4$ sur la transmission intergénérationnelle du
} statut de classe

\begin{abstract}
«Ma mère était ménagère, mon père était donanier. J'ai fait l'école primaire à Bonaké, le collège Adventiste de Bonaké, après Saint Viateur de Bonaké en comptabilité et puis après je suis venu à Abidjan j'ai fait 2 ans en entreprise en tant que comptable à la SIB, après je suis parti au lycée professionnel d'Odienné, puis l'INSET de Yamoussonkro pour mon BT. Après ma comptabilité, j'ai été employé comme chef magasinier à la bijouterie SABI ici à Treichville. Puis, j'ai changé je me suis transformé en chimiste au lycée professionnel d'Odienné en tant que technicien en qualité. Ensuite je suis reparti à l'INSET, après mon diplôme je suis venu j'ai été employé comme technicien assistant en laboratoire chez. CARGILL. C'est ce que je fais depuis plus de 15 ans ».

Extrait de l'entretien avec l'enquêté n4, 40 ans, technicien assistant de laboratoire, la taille du ménage est de 4 personnes.
\end{abstract}

Cette transmission intergénérationnelle passe notamment par les spécificités des parcours scolaires à l'image du cas de l'enquêté $n^{\circ} 4$. Le cas de Mr. H. (entretien $n^{\circ} 27$ ), 41 ans et auditeur comptable dans une société privée de publicité et de communication, est également représentatif de ces trajectoires. De père instituteur, il souligne que l'éducation a toujours représenté une priorité pour ses parents. Il a d'abord été scolarisé dans une école privée catholique, puis a ensuite suivi l'enseignement à l'école publique. Après l'obtention de son baccalauréat il obtiendra d'abord un BTS de comptabilité puis il effectuera une formation en Master audit, dans une école privée à Abidjan. A l'image des autres interviewés de cette catégorie, Mr. $\mathrm{H}$ a souhaité scolariser ses enfants dans un école privée (ici catholique). Il est très déçu du système d'éducation publique. Selon lui, «l'école est le parent pauvre de la Côte d'Ivoire »! Il n'attribue pas cette responsabilité 
à l'un ou l'autre régime depuis 20ans, mais à tous ceux qui ont suivis le régime d'HouphouëtBoigny. En prenant des exemples d'amis professeurs de chimie, il évoque le fait qu'ils n'aient pas de matériel pour faire leurs expériences ; une amie institutrice n'a même pas de craies pour le tableau et préfère les acheter elle-même ; et un ami directeur d'école a un budget de 3 millions de FCFA pour une école de 3000 élèves, qu'il reçoit en février, et pour lequel on lui demande de dépenser $80 \%$. Il dit ne pas envisager mettre ses enfants à l'Université publique, l'enseignement étant de mauvaise qualité, avec des grèves à répétition, et des conditions d'enseignement sousoptimales. Si les écoles privées poussent comme des champignons, c'est parce que cela répond à un besoin fort selon lui.

La diversification des revenus au sein de cette catégorie semble passer par des investissements dans les activités agricoles et l'immobilier. Le cas de Mr. H. est là aussi révélateur de ces pratiques et des capitaux économiques et socio-relationnels dont disposent les individus de cette catégorie de la classe moyenne. Mr. H. a donc plusieurs projets immobiliers : il a acheté 2 terrains, à Bingerville et à la Palmeraie, et ils ont souscrit, avec son épouse, à 2 projets immobiliers privés (en dehors des programmes présidentiels). L'un de ces derniers est effectué par le Lycée catholique Ste Marie à destination de ses professeurs (auquel ils ont pu se greffer) et l'autre est réalisé via la Direction Générale des Impôts. Si Mr. H. n'aime pas s'endetter il avait néanmoins pris un crédit immobilier pour l'achat de son terrain. Parce que la personne qui s'occupait de son dossier à la banque était une connaissance, à qui il avait donné des cours de gestion comptable, il a pu bénéficier d'un taux à $8 \%$ (au lieu de 15\%).

Enfin, on note dans ce groupe une attention et un temps plus important consacré aux loisirs, notamment pendant les weekends et les vacances annuelles (sorties à la plage, dans les bars, les activités sportives, les voyages). En termes de consommation, la fréquentation des grands supermarchés et malls y est relativement plus régulière. La fréquentation de ces lieux traduit également un comportement ostentatoire. Ainsi, Mr. O. (entretien n³0), âgé de 35 ans et agent administratif (secteur formel privé associatif), explique qu'il va avec son épouse, tous les $20 \mathrm{du}$ mois (date de leur anniversaire de mariage), prendre un café à Abidjan Mall (« juste un café, pour 3000 FCFA, ce n'est pas cher »), pour la beauté du cadre, sans y faire de shopping.

Le quatrième groupe est la classe moyenne des dirigeants, cadres et professions intermédiaires du secteur public. Il représente $17 \%$ de l'ensemble de la classe moyenne ivoirienne. Cette partie de la classe moyenne est composée de salariés occupant des postes de dirigeants et de cadres supérieurs et moyens du secteur public. Ils se démarquent par ailleurs très nettement par le fait qu'ils sont en moyenne beaucoup plus éduqués que les membres des autres groupes de la classe moyenne. On les retrouve nombreux dans l'administration publique et surtout dans l'éducation. Ils sont surreprésentés de manière générale en ville et en particulier à Abidjan où $26 \%$ d'entre eux résident. Contrairement à tous les autres groupes, ils ont très majoritairement accédé à leur emploi par des modalités d'accès autres que les relations personnelles. Les chefs de ces ménages de grande taille sont assez peu nombreux à posséder leur habitation, mais leurs revenus mensuels par habitant sont les plus élevés de tous les groupes. A l'instar du précédent groupe ils consacrent une part plus importante de leur revenu aux dépenses 
d'éducation. On retrouve dans les entretiens menés auprès des chefs de ménage de cette catégorie des trajectoires confirmant une forme d'héritage des positions sociales de leurs parents. Mais on observe aussi des trajectoires d'ascension sociale passant uniquement par le diplôme. Mme C. (entretien $\left.n^{\circ} 29\right), 37$ ans et fonctionnaire (conseillère d'orientation) explique avoir pu accéder à la fonction publique par ses propres moyens, via un «système méritocratique fonctionnel ». De père artisan et de mère ménagère, elle a fait ses études en sciences économiques à l'Université de Bouaké, avant que la crise ne l'oblige a migrer vers Abidjan où elle a suivi son cursus à l'Université de Cocody jusqu'à l'obtention de son DESS en économie et gestion. Les interviewés expriment également leur mécontentement au regard du système public d'enseignement. Les enquêtés $n^{\circ} 5$ et 10 , eux-mêmes acteurs du système scolaire et universitaire public, déplorent les problèmes de sureffectifs et leurs conséquences et ont ainsi choisi de scolariser leurs enfants dans le privé (encadré $\mathrm{n}^{\circ} 7$ ). Mme C. (entretien $\mathrm{n}^{\circ} 29$ ) souligne le manque d'école publique en Côte d'Ivoire et à Yopougon en particulier : sur cette commune de deux millions d'habitants, il existe selon elle seulement 4 établissements publics du secondaire. Elle déplore le pullulement de maquis par rapport au manque d'écoles, qui n'est pas à la hauteur d'un pays qui se dit émergent. Elle déplore également la faiblesse des salaires des professeurs, et les mauvaises conditions universitaires. Si ses moyens le lui permettaient, elle préfèrerait que ses enfants s'orientent dans une Université privée, pour la régularité de l'enseignement (éviter les grèves, les années blanches, etc.). Il n'est donc pas étonnant que son degré de consentement à l'impôt soit très faible : elle n'est pas prête à payer des impôts supplémentaires pour soutenir les politiques publiques, à part si son salaire est revalorisé.

\section{Encadré $n^{\circ} 7$ - Extrait d'entretien sur le positionnement de la classe moyenne des dirigeants, cadres et professions intermédiaires du secteur public à l'égard du système scolaire public}

La pluriactivité dans cette catégorie de classe moyenne s'exprime notamment à travers le statut d'agriculteur «absentéiste ». Les interviewés souligne qu'une partie important de leur épargne vient de ce type d'activité complémentaire. Un exemple nous est relaté dans l'entretien $\mathrm{n}^{\circ} 7$. En effet, ingénieur informaticien dans une entreprise à Abidjan, ce dernier investit dans les plantations de culture de rente héritées de son père (encadré $\left.n^{\circ} 8\right)$. 
Encadré $n^{\circ} 8$ - Extrait d'entretien sur les investissements agricoles de la classe moyenne des dirigeants, cadres et professions intermédiaires du secteur public

\begin{abstract}
"Cela dépend, par exemple, en tout cas le plus souvent, c'est les revenus qui viennent du champ qui constituent mon épargne... Je veux dire, hévéa (...). Quand on a vendu, ils virent l'argent dans un compte, je ne touche pas cela pendant un moment. Comme je l'ai dit, les $40 \%$ qui reste, et peut être à moitié 20\%, j'ai un compte credimatique où la banque prend pour garder ».

Extrait de l'entretien avec l'enquêté 7, 47 ans, de la catégorie de la classe moyenne de l'agriculture informelle. La taille du ménage est de 12 personnes.
\end{abstract}

De manière intéressante, il ressort des entretiens que les aspirations des interviewés sont assez nettement marquées par une volonté de promotion sociale et de prise en charge de son destin incluant une aspiration au statut de riche, renouant là avec cette volonté d'assimilation aux riches de la classe moyenne abidjanaise des années 1970-1980 (Touré, 1981 ; Le Pape, 1997). Ainsi l'enquêté $\mathrm{n}^{\circ} 9$, actuellement inspecteur du travail et des lois sociales dans l'administration publique, ambitionne une carrière de magistrat ou de diplomate. De même, l'enquêté $\mathrm{n}^{\circ} 8$, actuellement greffier au tribunal de Dabou, envisage également une carrière de diplomate et a déjà une idée claire du processus pouvant lui permettre d'atteindre son objectif. L'accès à la propriété en milieu urbain est également régulièrement souligné.

Le dernier groupe est celui de la classe moyenne des retraités et inactifs qui représente $\mathbf{1 5 \%}$ de l'ensemble de la classe moyenne. Cet ensemble regroupe principalement les ménages dirigés par des retraités ou des inactifs. Ils sont plus souvent que ceux des autres groupes dirigés par des femmes. Ces ménages sont souvent propriétaires de leurs logements et se distinguent par la faiblesse relative de leurs revenus, qui sont en moyenne les plus bas de la classe moyenne. Ce sont aussi ceux qui épargnent le moins en propension et en montant. Leur utilisation d'internet est plutôt plus faible que celle des autres et leurs dépenses sont marquées par les postes santé et éducation. Les entretiens qualitatifs auprès de ces ménages ont révélé des pratiques de diversification des revenus passant notamment par des investissements dans le foncier agricole. Les données quantitatives montrent d'ailleurs qu'ils sont plus de 30\% à être propriétaires de terres agricoles). Ils semblent également bénéficier de transferts de revenus de la part d'autres membres de la famille travaillant à Abidjan ou dans d'autres pays. Ainsi le cas de l'enquêté $n^{\circ} 6$ qui reçoit chaque deux mois un montant compris entre 50.000 et 75.000 FCFA de son fils installé aux Etats-Unis, tandis que sa fille nouvellement en fonction à Dakar lui fait parvenir 25000 FCFA par mois. Une autre fille résidant avec lui apporte 15000 FCFA chaque fin de mois et enfin une dernière exerçant dans une structure de communication lui fournit un crédit de communication mensuel de 10000 FCFA. La vulnérabilité de cette classe moyenne est également frappante (difficultés sérieuses au moindre choc, notamment en terme de santé). 


\subsubsection{La structure complexe des classes moyennes ivoiriennes: les enjeux socioéconomiques des processus}

de stratifications sociales en cours.

La notion de classe moyenne en Afrique et en Côte d'Ivoire repose principalement sur l'identification d'un mouvement global d'accroissement des revenus et d'augmentation de la consommation (des biens primaires et d'équipements, des biens scolaires et de santé) renforcé par des images «glamours» de malls et d'espace de consommation de type « occidental ». Pour autant, ces tendances globales cachent de profondes inégalités et la totale hétérogénéité des situations sociales concernées par ce processus (Melber, 2015, 2016 ; Giesbert et Schotte, 2016). Notre typologie permet ainsi de soulever quelques caractéristiques transversales traduisant autant d'enjeux spécifiques associés à ces classes moyennes ivoiriennes. Ainsi, les ménages rassemblés ne sont pas simplement différents en termes monétaires mais fortement hétérogènes en termes sociaux, culturels et politiques. Ils appartiennent à un milieu de revenus mais à des milieux sociaux forts différents. La figure $\mathrm{n}^{\circ} 4$ ci-dessous synthétise schématiquement les différences entre les groupes composant la classe moyenne ivoirienne du point de vue de leur niveau moyen de revenu, de leur degré d'informalité dans l'emploi (absence de comptabilité et d'enregistrement formels de l'activité) et du poids du salariat en leur sein. Elle est un bon point d'entrée pour discuter des enjeux autour des processus de stratifications sociales en cours associés à l'émergence de ces catégories de classes moyennes en Côte d'Ivoire.

Nos résultats montrent donc qu'en Côte d'Ivoire ce que l'on appelle classes moyennes recouvre plusieurs strates marquées par un fort dualisme (strates hautes vs strates basses) et sous-tendant des processus de stratification sociale et des comportements et aspirations différenciés.

Figure $n^{\circ} 4$ - L'hétérogénéité de la classe moyenne ivoirienne

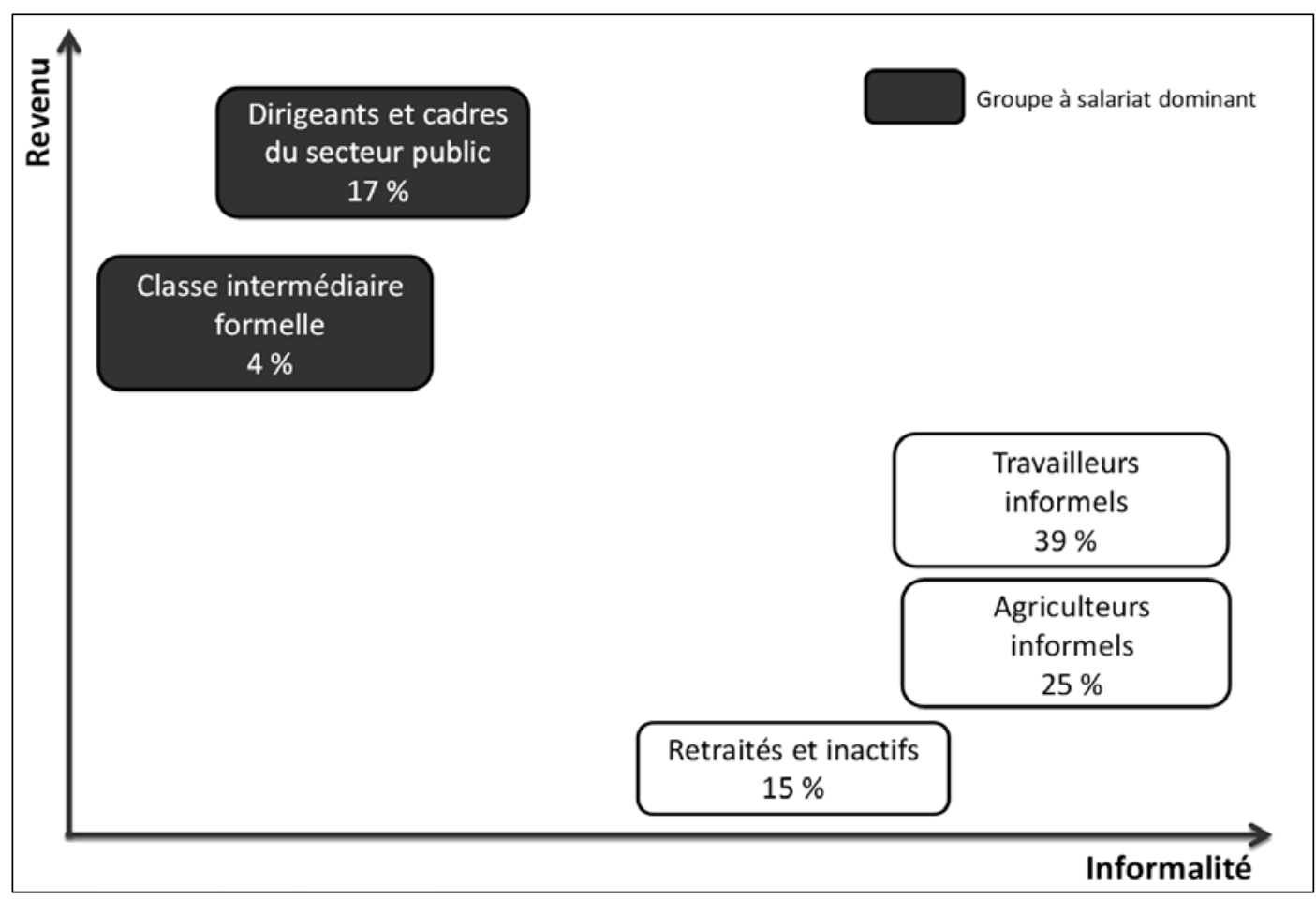

Source: Auteurs 
D'un côté, on retrouve en haut à gauche de la figure $\mathrm{n}^{\circ} 4$ des catégories représentatives de la strate haute de la classe moyenne et constituées plutôt d'héritiers des groupes intermédiaires des années 1960-1970. En dépit de la crise, ces catégories ont bénéficié d'un transfert intergénérationnel sur les différents types de capitaux nécessaires au succès (humain, économique et financier, relationnel et culturel). Il s'agirait plutôt d'un mouvement de réémergence de groupes caractérisés par leurs capacités d'intermédiations entre riches et pauvres, entre formel et informel, rural et urbain, salariés et indépendants. Le cas de Mr. H. (entretien $n^{\circ} 28$ ) est une bonne illustration de cette capacité d'intermédiation lui permettant d'accéder à différentes ressources dans le cadre de son activité professionnelle (primes cash des clients pouvant atteindre 5 millions de FCFA), ou en dehors (formation à la comptabilité pour des extérieurs, accès à du crédit bancaire «bon marché » via ses connaissances...). On retrouve ici la classe moyenne intermédiaire formelle et la classe moyenne des dirigeants, cadres et professions intermédiaires du secteur public. Elle est assez représentative de l'idée d'une classe moyenne africaine caractérisée par un emploi et un revenu stable (Strode et al., 2015). Dans le cas de la Côte d'Ivoire, cette strate est donc en partie héritière à la fois d'un transfert intergénérationnel effectif et d'un transfert symbolique par la stabilisation de pratiques sociales sur plus de vingt ans. Comprendre le thème de l'émergence des classes moyennes en Côte d'ivoire impose de revenir sur cette histoire des années 1950-1980 et d'identifier les liens des nouveaux émergents avec cette histoire récente. Dans notre étude statistique, nous identifions un groupe de " retraités et inactifs » particulièrement important (15\%) qui pourrait exprimer la permanence des groupes de classes moyennes en expansion des années 1960-1980 qui ont perduré mais avec des niveaux de revenus limités, et qui pourrait constituer une des expressions des capacités de transfert intergénérationnel des statuts sociaux. C'est un domaine qui doit impérativement donner lieu à des études spécifiques, et cela d'autant plus que ce groupe affiche aujourd'hui une très grande vulnérabilité et un niveau moyen de revenu par personne le plus bas de l'ensemble de classe moyenne faisant donc peser des risques sur capacité de transmission intergénérationnelle du statut de classe moyenne ${ }^{22}$. Si ces catégories de classes moyennes traduisent pour partie la permanence d'un stock, elles révèlent également en parallèle un mouvement d'aspiration à des compétences et à des savoir-faire spécialisés qui semble reprendre. Ce mouvement peut être de nature à relancer le processus d'accès direct à la classe moyenne pour les diplômés dans le secteur privé et public. Si le groupe des dirigeants et cadres du secteur public est significatif (17\%), celui du secteur privé est en revanche très réduit (4\%) payant directement le prix de la disparition massive d'entreprises du secteur moderne et d'emplois privés durant la décennie 2000-2010. Reste que ce groupe «emplois privés et publics du secteur moderne » confirme avec la reprise économique l'idée d'une nouvelle expansion de cet ensemble socio-professionnel validé par le diplôme, l'éducation et les compétences techniques. L'augmentation des rémunérations des salariés publics et privés

22 Le cas de $\mathrm{Mr} \mathrm{J}$ (entretien $\mathrm{n}^{\circ}$ 31) est à cet égard révélateur : pré-retraité de 64ans, ancien professeur de collège et de lycée, qui a gravi les échelons de l'administration publique (il est devenu Directeur d'un Cabinet Ministériel sous Laurent Gbagbo, pour ensuite être relégué au rang de professeur d'un lycée public à la chute du régime - ce qu'il a refusé) il affirme qu'auparavant, il se sentait appartenir à une classe moyenne, avec une identité de classe moyenne, mais que depuis $10 \mathrm{ans}$, il est sorti de cette classe, s'approchant davantage de la pauvreté. Mr J est aujourd'hui travailleur inactif, reculant le plus possible son passage effectif à la retraite, car redoutant un nouveau déclassement social. 
(notamment à travers la récente augmentation du SMIG), les opportunités de placements dans des plantations commerciales de type Héveas, et d'investissement dans le foncier urbain ainsi que les aides et passes droits consentis par l'administration semblent relancer un processus d'accumulation limitée au profit des classes moyennes qui dégagent des surplus significatifs. Ces catégories témoignent de comportements de consommation et de conditions de vie assez différentes de celles des strates basses. En terme de volume, l'effet revenu joue un rôle important, sur le plan des pratiques on note des lieux de consommation différenciés avec des fréquentions plus régulière des supermarchés et mall. Les enjeux politiques et économiques associés à cette catégorie concernent d'une part l'accès et la qualité de l'éducation et d'autre part la diversification du modèle de croissance ivoirien et sa capacité à générer des emplois formels de qualité.

D’un autre côté, un processus d'émergence de néo classes moyennes par le bas (la petite prospérité) semble également reprendre. Dans ce processus on va retrouver le groupe le plus important de la classe moyenne ivoirienne, celui des travailleurs de l'économie informelle urbaine (39\%). Ce groupe s'impose comme le résultat de tous ces processus en cours depuis la fin des années 1970 qui se sont traduit par le redéploiement des individus à capacités dans le petit secteur commercial et industriel informel pour échapper à la récession. Il est bien décrit dans la littérature passée (Contamin et Memel-Foté, 1995 ; Fauré et Labazée, 2000 ; cf. chapitre 1) et est aussi plus récemment identifié par Toh et Kouyaté (2009) à travers notamment des responsables de petites ou moyennes structures informelles liés aux télécommunications (cybercafé, centre de traitement informatique, centres d'appels et de vente de cartes téléphoniques, débits de boissons etc.). La récente étude UCP-UNILEVER (2017) sur la classe moyenne abidjanaise identifie également près de 38\% d'entrepreneurs indépendants. Les enjeux autour de cette catégorie sont centraux et ce phénomène devrait devenir une priorité d'analyse pour interpréter ses liens avec le secteur formel et le monde de l'administration et sa capacité à passer dans une logique d'intensification dans un projet global d'industrialisation et d'émergence du pays. En effet, si Kodila et al. (2014) voient dans l'informel une voie d'accès à la Upper Middle Class nos résultats témoignent d'une certaine précarité des conditions de vie des jeunes individus de cette classe moyenne avec néanmoins des aspirations réelles au devenir entrepreneurial. Il est donc difficile à ce stade de se prononcer sur la nature spécifique des dynamiques entrepreneuriales à l'œuvre dans ce secteur, entre entrepreneuriat de subsistance et entrepreneuriat d'opportunité (Ayadi and Ben Aïssa, 2015 ) ; et sur la capacité de ses entreprises à combler le "missing middle » du tissu productif ivoirien (Akinkugbe and Wohlmuth, 2016). Identifier les segments dynamiques de ces activités et comprendre les contraintes qui pèsent sur leur développement sont des sujets d'analyse de première importances. Accompagner ces activités dans un processus de promotion et d'amélioration des conditions de travail et de productivité est très certainement un préalable à leur formalisation (Berrou et Eekhout, 2018).

Dans cette dynamique d'émergence de néo classes moyennes par le bas, un dernier groupe fondamental pour l'économie ivoirienne présente une caractéristique spécifique par son caractère rural : c'est celui des agriculteurs (informels) notamment du sud-ouest. Ces derniers disposent d'un revenu significatif mais leurs capacités de consommation sont peu valorisées du fait d'une densité faible. La part du secteur agricole dans la formation de la classe moyenne ne doit pas 
surprendre dans un pays dans lequel ce secteur représente autour de $28 \%$ du PIB et a toujours constitué un élément majeur du régime de croissance. Le qualificatif «néo » est d'ailleurs ici à mettre entre guillemet tant cette catégorie a pu constituer une strate importante et protégée de la classe moyenne ivoirienne jusqu'à la fin des années 1980. Il est aussi à mettre entre guillemet compte tenu du fait que le vieillissement tant des planteurs que des plantations constitue un enjeu majeur de cette économie de plantation. Nos résultats témoignent par ailleurs de la vulnérabilité de ce groupe qui vit «selon la courbe», pour reprendre l'expression d'un des agriculteurs interrogés, et se sent un peu délaissé et oublié des pouvoirs publics. Aujourd'hui, ce système agricole (notamment d'exportation) ne présente plus les mêmes conditions d'ascension sociale, d'autonomisation et d'émancipation. Comme le souligne Balac (2016) «les jeunes ruraux ne rêvent plus, comme leurs parents et grands-parents, de gagner leur vie par le travail de la terre » (p. 57); ils se projettent plus comme des entrepreneurs individuels notamment hors du village. Les enjeux politiques et économiques associés à cette catégorie sont donc complexes et portent notamment sur la sécurisation de leurs conditions vie face aux différents risques auxquels ils sont confrontés : instabilités des prix, insécurité et tensions foncières dans un contexte de vieillissement des plantations, inégalités fortes dans la transmission intergénérationnelle.

Cette strate basse de la classe moyenne associant informels urbains et agriculteurs traduit donc une dynamique de stratification sociale qui ne peut que poser des questions majeures tant au regard de la vulnérabilité économique de cette classe aux chocs conjoncturels (cf. situation actuelle des prix du cacao) que sur les liens supposés immédiats entre montée des classes moyennes et renforcement et stabilisation des institutions (l'informel se construisant pour partie en dehors voir contre l'Etat) (Schotte, 2016 ; Resnick, 2015 ; Darbon et Toulabor 2014). Elle soulève aussi une autre logique de stratification sociale qui nous ramène plus vers cette partie "floating class» (BAD, 2011) de la classe moyenne qui structure une partie importante du débat autour des classes moyennes africaines. En effet, le buzz sur les classes moyennes africaines a entièrement reposé sur ce quiproquo autour de la "floating class » à la fois présentée comme différente de la classe moyenne mais pourtant intégrée dans les chiffres globaux de cette catégorie par la BAD (BAD, 2011). Notre étude a volontairement choisi de ne pas intégrer dans la catégorie classes moyennes les ménages au revenu par tête compris entre 2 et $4 \$$ pour des raisons liées à la trop grande vulnérabilité de ces populations (Thurlow et al., 2015) 23. Pour autant nos résultats nous forcent à constater que cette «floating class» s'articule probablement très bien avec nos deux groupes des informels urbains et des agriculteurs. Ce résultat souligne l'importance de passer d'une perspective en termes de stocks à une perspective en termes de flux. L'importance analytique de la «floating class» ne vient pas de son nombre mais de son processus de structuration et des mouvements que ses membres entretiennent avec la pauvreté. Elle est l'une des expressions de la sortie de pauvreté qui touche désormais aussi le continent africain et la Cote d'Ivoire et des orientations tendancielles des aller-retours que font les pauvres ou les «strugglers », entre situations de précarité et situations de petite prospérité (Darbon, 2012; Giesbert and Schotte, 2016). L'un des enjeux de l'avenir de la classe moyenne ivoirienne résulte dans l'accélération de ces flux et la raréfaction des retours en pauvreté. On passe alors sur des

\footnotetext{
${ }^{23}$ On notera que l'étude de la CFAO (2015) n'intègre pas non plus cette «floating class ».
} 
stratégies différenciées de la part des entreprises privées (solvabilisation de marchés BoP et articulation sur de nouvelles activités de production locale) et de l'Etat (mécanismes d'appui à l'émergence, de protections, de formation...) et des modes de production alternatifs ou complémentaires au modèle de développement par la seule expansion manufacturière. Ces catégories à la frontière basse des classes moyennes en sont une partie de l'avenir par le potentiel démographique qu'elles représentent. Une partie importante de l'enjeu de l'émergence ivoirienne se fait ainsi aussi par le bas de la pyramide avec ces individus en yoyo entre pauvreté et petite prospérité dont le destin fixera celui du « renouveau ivoirien ». Par ailleurs en termes politiques leur enjeu est important car à la différence des classes moyennes classiques, ces groupes s'expriment par et dans la rue, pas par leur inertie politique inexistante (Giesbert and Schotte, 2016).

Le travail d'interprétation de la nébuleuse « classes moyennes » ivoiriennes permet d'en préciser à la fois l'étendue, la structuration, les comportements et expressions de ses différentes composantes. Tout d'abord, si l'ampleur de la classe moyenne de revenu telle que nous l'identifions (4\$ - P95) est limitée à 26\% de la population ivoirienne, cela reste assez significatif au regard des standards de la région et comparables aux autres études existantes sur la Côte d'Ivoire (BAD, 2011 ; Ncube et Lufumpa, 2015 ; CFAO, 2015 ; UCT-UNILEVER, 2017). L'ensemble classe moyenne ivoirienne apparait aussi dans toute sa complexité. D'un côté dans cet ensemble de $26 \%$ de la population on peut identifier des traits communs : un même goût pour l'effort sur soi, pour la promotion de soi et le mérite ; une même importance accordée à l'éducation des enfants conduisant assez largement les membres de la classe moyenne à opter pour le secteur privé (dès lors que le secteur public est défaillant) ; un même effort soutenu d'épargne (en fonction des moyens disponibles) ; un même positionnement très individualiste, refusant les formes d'identité collective, se tenant à l'écart de l'Etat et des enjeux politique et se définissant par différenciation «ni riche ni pauvre ». D’un autre côté, l'ensemble apparait clairement dans toute son hétérogénéité dans cette étude, confirmant les orientations récentes de la littérature (Darbon et Toulabor, 2014 ; Melber, 2016 ; Schotte, 2017). L'analyse des cinq groupes principaux, soulignent à quel point ils se différencient sur un ensemble de variables qui les caractérisent (âge, occupation, niveau d'éducation, habitat, sexe, structure familiale, revenu, comportements de consommation et attitudes politiques et sociales). Au-delà de ces cinq groupes l'enquête montre les différences entre ménages les plus riches (strate haute) et les ménages relativement plus pauvres (strate basse), entre les ménages stabilisés en situation de gens du milieu (en stock) et les ménages vulnérables en situation de petite prospérité (en flux) ; entre ménages héritiers du milieu et néo-classes moyennes y accédant directement par leurs seuls efforts. Peu de choses relient ainsi ces différents sous-groupes au-delà de leur commun intérêt à poursuivre leur rêve d'un futur meilleur, et leur capacité à se projeter pour cela dans le futur. Les implications en termes de politiques publiques de cette stratification sont donc ambivalentes et demandent à être analysée plus précisément. 


\section{Les classes moyennes ivoiriennes : une classe promue, instrumentalisée, ou oubliée des politiques publiques?}

L'identification des classes moyennes en Côte d'Ivoire montre que cette catégorie est à la fois limitée en nombre ( $26 \%$ de la population), extrêmement hétérogène dans sa composition, diverse dans ses aspirations et fragile dans ses capacités de mobilisation collective. L'objectif du Président Alassane Ouattara d'une Côte d'Ivoire émergente d'ici 2025, supposant l'accroissement et la consolidation de ces catégories intermédiaires de revenus, se heurte à cette réalité et impose de soulever deux questions: existe-t-il véritablement des politiques publiques ciblant le développement et la promotion des classes moyennes ? Les politiques publiques répondent-elles aux attentes et aspirations de ces classes moyennes ? Pour répondre à ces questions, ce troisième chapitre repose sur l'analyse systématique des documents produits par les autorités politiques et sur un ensemble d'entretiens semi-directifs dits institutionnels avec les différents groupes d'acteurs intervenant soit dans la formulation de politiques publiques sectorielles, soit dans leur mise en œuvre (en tant qu'offreurs de biens et de services) (encadré $n^{\circ} 9$ et tableau $n^{\circ} 7$ ). Une première partie analysera le positionnement et les perceptions croisées des politiques publiques à l'égard des classes moyennes et inversement (3.1.). Dans une deuxième partie, la manière dont les politiques publiques tentent ou prétendent répondre aux aspirations et attentes des classes moyennes sera interrogée, et notamment au regard de trois secteurs spécifiques de politiques sectorielles pour lesquelles des attentes et/ou un ciblage classe moyenne semblent particulièrement affirmés (l'énergie, le logement et la couverture maladie/santé) (3.2.) ${ }^{24}$.

\subsection{Les politiques publiques et les classes moyennes en Côte d'Ivoire : un rendez- vous manqué?}

Comment les acteurs politiques perçoivent-ils les classes moyennes et, en retour, quelles sont les attentes de ces classes moyennes à l'égard des politiques publiques ? C'est à ces questions que nous essaierons de répondre dans cette section en abordant successivement: la manière dont l'objet « classe moyenne » est perçu du point de vue des acteurs des politiques publiques (3.1.1.), les attentes exprimées par les classes moyennes au regards de différents secteurs de politiques publiques (3.1.2), avant de conclure sur le constat de leur faible capacité à se faire entendre et leur faible engagement politique (3.1.3.).

24 Le choix de ces trois entrées sectorielles de politique publique s'appuie sur les résultats de nos entretiens institutionnels où ces dernières ont souvent été mises en avant comme illustratives d'une attention particulière portée aux classes moyennes. Elles permettent de plus de couvrir deux logiques de construction des politiques publiques selon qu'elles témoignent d'une ouverture plus forte aux opérateurs et financements privés (énergie, logement par exemple) ou qu'elles restent encore façonnées de manière significative par la coopération internationale (couverture sociale santé). 
3.1.1.1. La perception de l'objet classe moyenne du point de vue des acteurs politiques : des classes moyennes non ou mal identifiées?

Dans la sphère politique ivoirienne, la notion de classe moyenne est peu présente. Elle n'apparaît qu'une seule fois dans le Plan National de Développement 2016-2020, à la différence du concept omniprésent d'«émergence» économique, certes intimement corrélée à celui de classes moyennes, mais sans que ce lien soit clairement défini dans les discours politiques. L'image de la classe moyenne est très imprégnée de la vision des opérateurs privés, relayée par la presse internationale dans des articles aux titres accrocheurs tels que "La classe moyenne, nowvel horizon ivoirien » ou "A Abidjan, le réveil de la classe moyenne ${ }^{25}$. Cette vision essentiellement consumériste est en réalité une projection des classes moyennes occidentalisées (mode de consommation et de vie, caractère urbain et éduqué), qui concerne en réalité plutôt les $5 \%$ les plus riches et la strate haute et minoritaire de la classe moyenne ivoirienne que nous avons identifiée. La manière dont nos interlocuteurs lors des entretiens institutionnels ont défini en terme de revenus et caractérisés cette classe moyenne est assez révélatrice. Nombre d'entre eux ont effet une conception de la classe moyenne qui correspond plutôt à la classe riche. Ainsi, cet interlocuteur (entretien n ${ }^{\circ} \mathrm{S} 10$ ) pour qui les classes moyennes disposeraient d'un revenu compris entre 400.000 et 1.000 .000 FCFA, de biens immobiliers (« maison d'au moins 5 pièces ou un duplex »), auraient une pratique fréquente de loisirs (« sortie au restaurant et plage ») et logeraient essentiellement à Abidjan dans des quartiers tels que ceux de la commune de Cocody ("Angré, Riviera, ancien II Plateau, Cocody centre »). Sur la base de ces critères, cette strate regroupe, selon lui «des cadres d'entreprises privées et publiques, des hommes d'affaires, des médecins et des enseignants du supérieur». On retrouve des définitions monétaires de la classe moyenne assez similaires dans plusieurs de nos entretiens. Notre interlocuteur dans le cadre de l'entretien n ${ }^{\circ} \mathrm{S} 11$ estime le potentiel d'épargne mensuel d'un individu de la classe moyenne à « $250.000-500.000$ FCFA».

25 «La classe moyenne, nouvel horizon ivoirien » par Maria Malagardis, Libération (23 octobre 2015); «A Abidjan, le réveil de la classe moyenne », Marie de Vergès, Le Monde (18 février 2017) 


\section{Encadré $n^{\circ} 9$ - La méthodologie des entretiens institutionnels}

Ces entretiens qualitatifs auprès de représentants d'institution et d'opérateurs privés ont reposé sur la méthode de l'entretien semi-directif. La consigne générale portait : "Nous soubaiterions donc échanger avec vous sur la façon dont votre institution perçoit ce phénomène d'émergence des classes moyennes et comment elle se positionne par rapport à celui-ci $\gg$. La grille de thème couvrait ensuite essentiellement la perception et le positionnement de l'institution au regard du phénomène de l'émergence des classes moyennes. Ces entretiens rentrant dans la catégorie spécifique des entretiens auprès des élites, cette grille devait restée très souple et évolutive et s'adaptée en fonction du type d'institution ou d'organisation enquêtée.

L'objectif de ces entretiens institutionnels est d'apporter un éclairage d'échelle méso, complémentaire au cadrage macroscopique du pays et aux entretiens ménages. Confronter les informations obtenues auprès des acteurs des politiques publiques et des opérateurs des stratégies privées à celles obtenues auprès des ménages de la classe moyenne permet notamment de vérifier la cohérence entre les aspirations des cibles de l'action publique et les offres politiques qui leur sont proposées. Les secteurs enquêtés, présentés dans le tableau $n^{\circ} 7$ et numérotés de S1 à S12, sont pour partie le fruit des disponibilités des interlocuteurs et surtout de l'importance relative des secteurs concernés dans la société et l'économie du pays.

Tableau $n^{\circ} 7$ - Les secteurs institutionnels enquêtés*

\begin{tabular}{|c|c|c|}
\hline $\begin{array}{c}\text { Secteurs } \\
\text { Institutionnels }\end{array}$ & $\begin{array}{l}\text { Entretiens } \\
n^{\circ}\end{array}$ & Institutions enquêtées \\
\hline \multirow{2}{*}{ Education } & S1 & Ministère de l'Education Nationale \\
\hline & S2 & Groupe Pigier \\
\hline \multirow{2}{*}{$\begin{array}{l}\text { Mobilité, } \\
\text { connectivité }\end{array}$} & S3 & Ministère du Transport \\
\hline & S4 & SOTRA \\
\hline \multirow{2}{*}{$\begin{array}{l}\text { Marché du travail et } \\
\text { Protection sociale }\end{array}$} & S5 & Ministère de l'emploi et de la protection sociale \\
\hline & S6 & Caisse Nationale de Prévoyance Sociale (CNPS) \\
\hline Genre & S7 & $\begin{array}{l}\text { Ministère de la femme de la protection de l'enfant et } \\
\text { de la solidarité }\end{array}$ \\
\hline \multirow[b]{2}{*}{ Energie et Habitat } & S8 & $\begin{array}{l}\text { Ministère de la Construction, du Logement, de } \\
\text { l'Assainissement et de l'Urbanisme }\end{array}$ \\
\hline & S9 & $\begin{array}{l}\text { Experts en foncier urbain (cabinet d'avocats et } \\
\text { chercheur en Science Politique - Université } \\
\text { Houphouët Boigny) }\end{array}$ \\
\hline Sécurité/démocratie & $\mathrm{S} 10$ & Groupe de sécurité privée G4S Secure Solution \\
\hline \multirow{2}{*}{$\begin{array}{l}\text { Secteurs } \\
\text { transversaux }\end{array}$} & S11 & Ministère du Plan et du Développement \\
\hline & S12 & PNUD \\
\hline
\end{tabular}

* Pour des raisons de confidentialité nous ne powvons préciser les interlocuteurs enquêtés qui dans leur ensemble occupaient tous des postes à haute responsabilité au plus haut de la biérarchie des institutions concernées et étaient donc à ce titre des interlocuteurs privilégié car disposant des informations nécessaires au regard de notre sujet. 
Sous l'hypothèse d'un taux d'épargne de 15-20 \% cela conduit à des niveaux de revenus bien supérieurs aux bornes monétaires de notre classe moyenne. Si le ministre du commerce reprend en partie cette vision, il en propose une interprétation plus inclusive en déclarant le 24 mai lors d'une visite sur le chantier du nouvel hypermarché huppé Playce (route de Bingerville) : "Je suis heureux de l'avancement des travaux qui se font à PlaYce Palmeraie Cocody (...) Tout ceci vient stimuler en fait la concurrence dans le secteur des distributeurs de produits alimentaires, des distributeurs de divers produits de consommation, qui sont destinés soit à la classe moyenne, soit à la majorité des ivoiriens »; ainsi, "la Côte d'Ivoire continue sa marche dans une dynamique d'aller de l'avant par rapport aux ambitions que le Chef de l'Etat s'est fixé pour faire de la Côte d'Ivoire, un pays émergent " ${ }^{26}$. L'élargissement du périmètre de la classe moyenne à la «majorité des ivoiriens » traduit ici la volonté d'orienter les investisseurs vers des marchés nouveaux en cours de solvabilisation, constitués encore aujourd'hui de ménages aux revenus modestes mais qui commencent à aspirer à des formes de consommation primaire plus élaborées. On voit ainsi apparaitre clairement des représentations différentes des classes moyennes en fonction des horizons temporels.

Une partie important de la classe moyenne identifiée dans notre étude semble en fait plutôt considérée comme pauvre par les décideurs politiques. Les politiques publiques sont ainsi paradoxalement clivées entre des politiques de lutte contre la pauvreté, notamment impulsées par les bailleurs internationaux (politiques "pro-pauvres »), et des ambitions d'émergence à l'horizon 2020, via une ouverture aux investisseurs privés supposée bénéficier à l'ensemble de la société (PND 2016-2020), mais qui touche réellement très peu les « classes moyennes » telles que nous les définissons. Pourtant nous observons bien dans nos données quantitatives, comme qualitatives, des attentes légitimes de ces classes moyennes sur différents secteurs de politiques publiques, des attentes potentiellement différenciées de celles des populations plus pauvres (et des plus riches) mais qui peinent à se faire entendre faute de traduction politique évidente de ces revendications.

\subsubsection{De fortes aspirations socioéconomiques: les attentes légitimes des classes moyennes dans plusieurs secteurs de politiques publiques}

\section{L'éducation et la santé au cour des préoccupations}

L'éducation apparaît comme le secteur qui cristallise le plus d'attentes chez les classes moyennes. Les acteurs interrogés dénoncent unanimement les errements du système public d'éducation et l'absence de politiques tournées vers l'avenir, depuis le primaire jusqu'au supérieur : baisse de la qualité de l'éducation, politiques de communication du gouvernement (« on ne forme pas avec des slogans !» dénonce $\mathrm{Mr} \mathrm{J}$., entretien ménage $\mathrm{n}^{\circ} 31$ ), absence de politiques de recherche (" autant Ouattara ne peut pas faire grand-chose sur les cours des matières premières, pour le pétrole ou le cacao, mais il peut faire le choix d'investir dans la recherche. C'est mon cri du cour! ", Mr O., entretien ménage $\mathrm{n}^{\circ}$ 30), mauvaises conditions matérielles, ou encore instabilité de l’Université (années blanches,

\footnotetext{
${ }^{26}$ Cf. https://cnlvc.ci/2017/05/31/visite-ministre-commerce-souleymane-diarrassouba-a-playcepalmeraie-ouverture-prochaine/, page consultée le 13/11/2017.
} 
grèves à répétition). Nombre d'interviewés font le choix du privé, et notamment du privé confessionnel, réputé pour une meilleure qualité de l'enseignement que d'autres structures privées, parfois apparentées à des commerces et peu réglementée par l'Etat. Aussi, tandis que beaucoup ont pu s'élever socialement, grâce à l'éducation et à un système méritocratique relativement fonctionnel (octroi de bourses, accès aux concours administratifs), ils sont plus pessimistes quant à l'avenir des générations futures.

De la même manière, la plupart des personnes interrogées dénoncent la qualité des structures de santé publiques (accueil, temps d'attente) et se tournent, lorsqu'ils en ont les moyens et qu'ils ont pu souscrire à une assurance maladie, vers des structures privées. Ils estiment que le projet de CMU du Président Ouattara est en soi, un très bon projet, et se disent globalement prêts à contribuer aux 1.000 FCFA mensuels par tête, bien qu'ils demeurent circonspects sur sa mise en œuvre effective.

\section{L'emploi : la recherche d'accroissement et de stabilité des revenus dans un contexte de rareté de l'emploi formel}

Les classes moyennes sont à la recherche de meilleurs revenus et de stabilité. Dans un contexte où les opportunités d'emplois formels sont rares, les catégories de la strate basse de la classe moyenne ivoirienne voient dans l'entreprenariat une opportunité de stabilisation professionnelle, quelle que soit sa forme (création d'un petit commerce, d'une entreprise, activités de consultance, etc.). Du côté des strates hautes, l'entrepreneuriat représente une source de diversification des revenus permettant d'être moins dépendant de son seul emploi salarié (autonomisation). Les interviewés n'évoquent que très rarement l'emploi salarié comme projet ou ambition future pour plus de stabilité. Cela souligne à quel point les opportunités sont rares et soulève en même temps un paradoxe pour ces classes moyennes en devenir, où l'entrepreneuriat, par définition plus risqué et instable, devient la seule porte de sortie. Dans le même sens, la fonction publique n'est plus perçue comme une forte opportunité pour s'assurer un train de vie confortable (avantages en nature type logements supprimées, évolutions de carrières bloquées), ce qui présente un changement radical avec les opportunités qu'elle représentait pour les générations précédentes.

\section{Les besoins en transport et infrastructures routières}

Le transport est un enjeu central pour des classes moyennes qui habitent des quartiers excentrés du centre d'Abidjan afin de bénéficier d'un loyer abordable. Les déplacements sont problématiques (l'autoroute unique reliant le centre d'Abidjan à Yopougon commune typiquement classe moyenne de 2 millions d'habitants est saturée pendant $2 \mathrm{~h}$ à $4 \mathrm{~h}$, matins et soirs). Mme C. (entretien ménage $n^{\circ} 29$ ), de Yopugon, met de $1 \mathrm{~h}$ à $1 \mathrm{~h} 30$ pour se rendre à son travail ce qui est peu par rapport à ceux dont l'activité professionnelle se situe à l'extérieur de la commune. Les nouvelles infrastructures (3ème pont à péage, nouvelles voies rapides de la Riviera Golfe, route de l'aéroport, Marina sur la lagune) sont perçues comme une vitrine de l'émergence, concentrées sur les quartiers riches. La construction du pont de Yopougon est attendue avec impatience. L'acquisition d'un véhicule personnel n'est généralement pas considérée comme une 
aspiration première, en particulier pour les strates basses, ce dernier étant hors de leur portée (prix d'achat très élevé en raison des taxes douanières, assurance, entretien, essence, etc.), les usagers faisant avec une diversité extrême d'offres de transports collectifs. Ces enjeux posent la question de la soutenabilité d'un modèle de ville essentiellement horizontal.

\section{Fiscalité et redistribution : une dénonciation commune des inégalités}

Nos entretiens auprès des ménages ont révélé à quel point les classes moyennes dénoncent de manière quasi générale le creusement des inégalités sociales et l'enrichissement flagrant des plus riches, via une corruption perçue comme inflationniste. Par exemple, Mr J. (entretien ménage $\mathrm{n}^{\circ} 31$ ) dénonce : "il faut regarder le train de vie de l'Etat! S'il est revu à la baisse, on pourra améliorer beaucoup de choses. Est-ce qu'on a besoin d'avoir cinq voitures, parce qu'on est Directeur de ceci ou de cela? Vous ne pouvez. pas vous diviser en cinq!». De même, Mr. H (entretien ménage $\mathrm{n}^{\circ} 27$ ) fait le constat amer d'une "Côte d'Ivoire à deux vitesses ». Pour lui, le pays se sépare en deux blocs : «ceux qui profitent du système, et ceux qui s'accrochent ». Ainsi, le consentement à l'impôt est plutôt faible, en raison de la faible légitimité de l'Etat et de l'impression d'un faible retour sur ces impôts.

\section{Les enjeux de la sécurité dans le contexte de réconciliation nationale.}

Le traitement de la résolution du conflit et le sentiment d'une « justice des vainqueurs », laissent couver un sentiment de rancœur, bien que la peur d'une nouvelle crise politique tende à favoriser la position de status quo des classes moyennes. L'apparition du phénomène " microbes ", des enfants et adolescents (8-25ans) qui agressent en bandes organisées, inquiète la plupart des ménages interrogés. Ce phénomène est né au moment de la crise post-électorale à Abobo, un quartier pauvre d'Abidjan, et tend à gagner les quartiers occupés par les classes moyennes (Yopougoun, Angré), où ils sévissent de plus en plus. Les populations classes moyennes perçoivent une forme de démission de l'Etat, et un sentiment d'insécurité renforcé par une faible confiance dans la police. Le documentaire « Faire l'incroyable, parole aux enfants dits 'microbes' » réalisé par le sociologue Francis Akindès, montre à la fois ce phénomène et le sentiment de précarité vécu par des familles classes moyennes menacées par un retour dans la précarité.

\subsubsection{Le faible engagement politique des classes moyennes ivoiriennes}

En Côte d'Ivoire, pas plus que dans la quasi-totalité des pays africains, la notion de classe moyenne ne peut prendre un sens sociologique et politique articulé autour de l'idée d'une conscience d'appartenance collective.

La question de l'identification des individus à cette catégorie est d'autant plus difficile à analyser qu'elle fait l'objet de discours systématiquement répétés qui altèrent la manière dont les individus se perçoivent. L'a-priori très positif dont elle est parée amènent les riches à l'adopter pour se débarrasser des qualificatifs négatifs qui sont accrochés le plus souvent à cette petite partie de la population dont une partie significative de la richesse provient d'un système de gouvernement 
néo-patrimonial et clientéliste consacrant des inégalités particulièrement fortes. Aussi, au sein des 5\% les plus riches une forte disparité existe (Cogneau et Czajka, 2016) de sorte que le bas de cette catégorie (les percentiles 95-96 par exemple) se vit comme "classe moyenne» au regard des niveaux de richesse démesurés des $1 \%$ les plus riches. Si l'on peut considérer qu'une partie de la classe moyenne peut également tendre à éviter cette qualification pour ne pas attirer l'attention sur sa richesse relative (pressions familiales et communautaires, peur des agressions), dans l'ensemble elle ne s'y identifie tout simplement pas. Le plus souvent notre population (4 US\$ P95) se définira par le "ni-ni », ni riche, ni pauvre, signifiant non pas qu'on se trouve au milieu, mais un rejet total de la pauvreté et des pauvres dont on vient et avec lesquels les flux de retour dans la pauvreté sont devenus très rares et une attraction pour ce qui est impossible pour l'instant, la catégorie des riches. Pour autant ce «ni-ni»n'a pas permis de constituer une catégorie en soi et pour soi. En dépit de son ancienneté et en partie en raison de la crise économique et politique des années 1980-2000, la catégorie moyenne en Côte d'Ivoire n'a pu se stabiliser autour d'un noyau dur d'héritiers stabilisant des manières de faire.

L'engagement politique des classes moyennes ivoiriennes est donc peu différent du constat global fait par l'OCDE (2016a, p148) qui, à travers le sondage réalisé par Gallup, souligne qu' en moyenne, $65 \%$ des personnes interrogées se sentent indifférentes à la vie publique, un taux nettement supérieur à la moyenne des pays africains». Le rapport constate que "ce manque d'engagement des citoyens dans la vie politique est conforté par les chiffres d'Afrobaromètre qui sonde la prise de contact des citoyens avec des représentants du pouvoir local et national (...). En 2013, 90 \% des Ivoiriens n'ont jamais contacté un représentant du pouvoir local et ce chiffre monte à $96 \%$ quand il s'agit d'un représentant du pouvoir national ». Les résultats de nos entretiens ménages montrent aussi que ces classes moyennes sont essentiellement mues par la recherche d'intérêts personnels et assez matériels (conditions de vie, logement, sécurité...) et la protection de leurs stratégies de promotion personnelle et familiale. Avec ces types de classes moyennes les mobilisations collectives sont très limitées et l'altruisme politique réduit. En termes de politiques publiques, cela signifie notamment que ces catégories de classes moyennes vont donc avoir tendance à se recroqueviller sur leurs avantages reconquis et revendiquer le retour de leur prospérité passée et avantages anciens comme le montrent les revendications salariales du privé et des militaires en Côte d'Ivoire. Il n'y a derrière ces comportements aucune attitude générale favorable ou défavorable en soi à la démocratie, à un gouvernement modéré et à des pratiques de marché transparentes remettant en cause la corruption. II y a des positions purement opportunistes, non altruistes, visant à assurer le retour du processus d'enrichissement individuel. Cela signifie donc aussi que ces catégories soutiendront tout ce qui facilitera leur vie et assurera la promotion de leur situation. En revanche tout ce qui remettra en cause leur nouveau mode de vie, et mieux ou pire les emblèmes de ce nouveau mode de vie (automobiles, transports, ...) sera systématiquement combattu. Toutefois, en Côte d'Ivoire, " la classe moyenne » a la particularité d'avoir été échaudée par les coûts de la crise politique et militaire, ce qui devrait favoriser dans l'ensemble une tendance au status quo, c'est-àdire une tendance légitimiste dominante commune à tous les groupes dès lors que le pouvoir garantit le retour à la stabilité et l'accès à la prospérité. 
Mais, au-delà de cette situation particulière, les différents groupes de classe moyenne peuvent avoir des intérêts divergents en termes de politiques publiques. A titre d'exemple, en termes de politiques de transport cela peut se traduire par des conflits d'intérêts entre les groupes de petite prospérité très favorable au transport en commun améliorés et les Upper Middle Class plus favorable au tout voiture individuelle. Ces groupes hétéroclites et hétérogènes sont ainsi rarement en position de se mobiliser de manière unie et active. Les strates hautes des classe moyennes peuvent être davantage favorables au statu quo et à la stabilité (d'autant plus si leur position dépend de l'administration en place et des coalitions au pouvoir). Néanmoins, le creusement des inégalités dans le haut de la distribution des revenus (les $5 \%$ les plus riches) peut être de nature à nourrir des frustrations relatives. Ainsi, en est-il pour Mr. J. ancien haut cadre la fonction publique (entretien ménage $\mathrm{n}^{\circ} 31$ ) pour qui «l'ostentation est de mise ». Pour lui, les inégalités sociales sont criantes : le nombre de personnes qui bénéficient d'un logement est faible, et l'accès au logement est impossible. De même, Mr. H. membre la strate haute de la classe moyenne du secteur privé formel (entretien ménage $\mathrm{n}^{\circ} 27$ ) dénonce ces inégalités ("chaque peuple a une capacité limitée à encaisser. (...) Une classe roule avec des voitures à $50 M$ de FCFA pendant que les gens s'appauvrissent. Les injustices sont trop criardes») et le manque d'espoir collectif de la société ivoirienne ("l'avenir est obstrué par des gens qui ne pensent qu'à eux»). Quant aux strates basses, qui pourraient être davantage portées au changement (Wietzke and Sumner, 2014), leur capacité de mobilisation politique est minime. En réalité ces dernières adoptent davantage des positions de « survivalists » et de «strugglers ", développant des stratégies purement individuelles.

\subsection{Des politiques publiques répondant aux attentes des classes moyennes ? Entre problème de ciblage, promotion et instrumentalisation des classes moyennes}

Au regard des éléments précédents, il s'agira, après avoir présenté de manière transversale un ensemble de politiques publiques sectorielles ciblant peu les classes moyennes (3.2.1.), d'analyser de manière plus approfondie trois secteurs de politiques publiques. Le premier, celui de l'énergie, illustre parfaitement comment l'absence de réponse aux attentes des classes moyennes peut conduire à des débuts de contestation portées notamment par les strates hautes (3.2.2). Les deux autres, le logement (3.2.3) et la couverture santé maladie (3.2.4) si elles affichent un ciblage pouvant répondre aux attentes différenciées des strates hautes et basses des classes moyennes peinent dans la pratique à y répondre.

\subsubsection{Des politiques publiques très peu ou indirectement ciblées sur les classes moyennes}

\section{Les politiques publiques de l'éducation : les classes moyennes délibérément oubliées?}

Depuis le milieu des années 1980, le système éducatif ivoirien s'est fortement dégradé, jadis perçu comme un des fleurons éducatifs de l'Afrique de l'ouest. Aujourd'hui, les indicateurs sont au rouge : au niveau quantitatif, l'espérance de vie scolaire n'est que de 7,7 ans, comparativement à une moyenne africaine de 9,7 ans, et de 12 ans pour les pays à revenus intermédiaires en 2015 ; au niveau qualitatif, une étude réalisée en $3^{\text {ème }}$ année du primaire en 2012 montrait que $87 \%$ des 
élèves ont un niveau faible ou très faible en français et $73 \%$ en mathématiques, avec une forte détérioration entre 1996 et 2009 (Banque Mondiale, 2017; OCDE, 2016a). Depuis 2010, le budget de l'Etat pour l'éducation augmente de 7,5\% par an, consacrant $5 \%$ de PIB à l'éducation, avec des chantiers concernant la construction et la réhabilitation des infrastructures (bien que souvent critiquées pour leur caractère « au rabais », telle que pour l’Université Félix HouphouëtBoigny dont les travaux se sont élevés à 130 milliards de FCFA), ainsi que l'embauche d'enseignants, l'octroi de bourses pour les étudiants de $2^{\text {ème }}$ et $3^{\text {ème }}$ cycles. Cependant, si la Côte d'Ivoire souhaite devenir un pays émergent, la Banque mondiale (2017) estime qu'elle devra doubler son budget lié à l'éducation d'ici 2025 et passer à la vitesse supérieure pour rattraper le retard accumulé. Dans ce contexte, les classes moyennes ne semblent pas constituer une cible particulière des politiques publiques. La priorité est clairement, dans un contexte de rareté des ressources, à la lutte contre la pauvreté, l'accès à l'éducation pour les plus pauvres. Comme le souligne notre interlocuteur dans le cadre de l'entretien $\mathrm{n}^{\circ} \mathrm{S} 1$ : "J'aurai tort de cibler ces catégories. Parce qu'elles se débrouillent suffisamment déjà. Ce que je veux dire c'est qu'elles tirent le meilleur de ce qui existe déjà ». Les établissements privés constituent alors pour le gouvernement une opportunité pour pallier le déficit de l'offre éducative. A tire d'exemple, 75\% des bacheliers de la session 2017 ont été affectés dans les grandes écoles privées ${ }^{27}$. L'offre privée s'oriente de facto vers ces ménages capables de subvenir aux frais de scolarité, souvent en sacrifiant une partie de leur bien-être ou de leurs projets d'investissements (entretien $\mathrm{n}^{\circ} \mathrm{S} 2$ ). La question de l'adéquation du système éducatif et de formation aux réalités économiques du pays et aux besoins des entreprises est aussi un des enjeux soulevés par nos différents interlocuteurs à ce sujet (entretiens nº S1 et S2).

\section{Les politiques de l'emploi: en attendant les effets du changement structurel, quel soutien aux indépendants non agricoles et agricoles?}

La manière dont les politiques de l'emploi contribuent à la promotion et la sécurisation des classes moyennes repose sur plusieurs leviers d'action ciblant plus ou moins directement ces catégories sociales. D'une part, une partie de ces politiques contribue à consolider le statut des salariés du secteur formel à travers des mesures comme la revalorisation récente du SMIG. Ensuite, les objectifs de création d'opportunités d'emplois salariés formels de meilleur qualité dépendent très largement des enjeux associés à la transformation structurelle de l'économie ivoirienne (diversification, montée en gamme...) (Banque Mondiale, 2015b ; OCDE, 2016a, b, c). Le développement du secteur privé formel et des petites et moyennes entreprises dépend par ailleurs de conditions relatives à l'amélioration du climat des affaires et au financement de l'économie via des réformes du secteur bancaire afin notamment d'assurer une meilleure captation de l'épargne privée (Ehrhart, 2015). Dans ce cadre, les classes moyennes sont surtout de potentiels bénéficiaires indirects de politiques sectorielles industrielles, agricoles et commerciales. Dans cette perspective, le ciblage des classes moyennes du point de vue des politiques de l'emploi passe donc principalement et peut être prioritairement par le soutien aux dynamiques entrepreneuriales portées par les indépendants et les petits patrons de l'informel non agricole et agricole. Il est difficile de se prononcer catégoriquement sur la portée et la nature

\footnotetext{
${ }^{27}$ https://news.abidjan.net/h/624753.html
} 
spécifiquement entrepreneuriales des dynamiques ici à l'œuvre, tant le secteur informel est hétérogène et recouvre des situations allant de l'entrepreneuriat de subsistance à l'entrepreneuriat d'opportunité. Si nos résultats témoignent d'une certaine précarité de leurs conditions de vie, ces indépendants et leurs micro-entreprises se situent à des niveaux de revenus qui les éloignent pourtant nettement du seuil de pauvreté. Il n'est donc pas impossible que cette classe moyenne des entrepreneurs informels recouvre pour partie celle des "constrained gazelles" identifiées par Grimm et al. (2012) et qui pourrait à terme combler le «missing middle » du tissu productif ivoirien. On peut en effet aisément considérer que le sens de l'entrepreneuriat de cette catégorie des classes moyennes est bien présent, ne serait-ce que dans sa capacité à jouer de la pluriactivité dans une logique d'accumulation extensive, mais aussi dans les comportements d'épargne et d'investissement, dans la prise de risque, etc. Seulement, ce comportement entrepreneurial s'exprime dans un environnement particulièrement instable et contraint. L'objectif des politiques publiques consiste alors à lever les contraintes auxquelles font face ces micro entreprises dans un processus graduel et équilibré maniant promotion (soutien à l'activité et à l'accroissement de la productivité) et formalisation (fiscalisation et respect des normes de travail) (Berrou et Eekhout, 2018 ; FMI, 2017). Les entretiens institutionnels nº 55 et $\mathrm{S} 6$ ont permis d'identifier des initiatives en cours qui s'inscrivent dans cet objectif. Par exemple, l'amnistie fiscale décrétée par les autorités ivoiriennes au cours de l'année 2016 pour inciter les entreprises informelles à s'enregistrer. Selon le ministre en charge du budget cette initiative porte ses fruits puisque "5462 entreprises sont venues se déclarer et ont été réintégrées dans le système ${ }^{28}$. Pour autant cela ne saurait être suffisant. Les contraintes qui pèsent sur le développement de ces micro entreprises renvoient également à des problèmes de compétences (éducation et formation), d'accès au crédit, d'accès aux infrastructures (transports, électricité) et aux insécurités et instabilités foncières et administratives. D'autres initiatives, relatives notamment au développement de produits de couverture sociale pour les travailleurs indépendants du secteur informel, tardent à se concrétiser. Deux régimes de prévoyance et de retraite à destination des travailleurs indépendants (avec un régime de base incluant des prestations de retraites et d'indemnités journalières et un régime de retraite complémentaire par capitalisation pour les indépendants dont les capacités contributives sont plus élevées) sont en effet à l'étude ${ }^{29}$. Notons par ailleurs, que pour les agriculteurs informels certains enjeux sont spécifiques aux filières agricoles concernées et portent notamment sur leur exposition à l'instabilité des cours internationaux des matières premières dans le cadre de filières libéralisés.

Enfin, face à ce double défi de la modernisation et de la formalisation des entreprises informelles, le développement rapide des technologies de l'information et de la communication dans le pays est de nature à offrir de nouvelles perspectives aux acteurs économiques. L'explosion des usages numériques, et notamment du mobile, pourrait soutenir la dynamique des unités de production informelles de multiples manières (Berrou et al., 2017 ; Berrou et Mellet, 2017 ; Le Quentrec,

28 «Environnement des affaires : Le ministre du Budget reconnaît une pression fiscale sur les entreprises ", Publié le vendredi 23 decembre 2016 | Le Quotidien d'Abidjan. Consulté le 4/11/2017: http://news.abidjan.net/h/606604.html

29 «Côte d'Ivoire : trois nouveaux régimes de prévoyance sociale d'ici fin 2018 » par E. Atcha | La Tribune Afrique, 19/05/2017 | Consulté le 15/11/2017:

http://afrique.latribune.fr/politique/politique-publique/2017-05-19/cote-d-ivoire-trois-nouveauxregimes-de-prevoyance-sociale-d-ici-fin-2018-716935.html 
2017 ; Rousset et Zoro, 2017). Avec un mobile GSM simple, il est aujourd'hui possible de négocier avec des fournisseurs ou des clients, de recevoir des informations sur les prix des matières premières ou encore de transférer de l'argent et de recevoir des paiements. Avec un smartphone, il est possible d'animer une page Facebook ou une page web commerciale pour y présenter ses produits et services, de partager des photos et des vidéos, d'avoir des conversations avec des groupes d'utilisateurs via la messagerie instantanée, de consulter des vidéos de tutoriels ou de s'informer sur l'état du marché.

Les politiques fiscales: des systèmes de redistribution communautaires aux systèmes de redistribution public, le risque d'une pression excessive sur les classes moyennes

Du point de vue des politiques fiscales les priorités affichées sont clairement à la consolidation de l'administration fiscale et au renforcement de l'efficacité de son fonctionnement (OCDE, 2016b, c). Pour autant, l'intégration des enjeux relatifs aux classes moyennes dans l'extension et la consolidation des systèmes fiscaux ne devrait pas être négligé. En effet, si pour l'heure les classes moyennes ivoiriennes restent en grande partie en dehors des régimes fiscaux et du système de redistribution formels (exception faite des salariés du formel), elles sont néanmoins soumises à la fiscalité indirecte. Au regard des défaillances actuelles des services publics qui leur sont potentiellement dédiés, leur intégration progressive aux systèmes formels de redistribution pourrait contribuer à renforcer leur sentiment d'être "corvéable à merci» pour reprendre l'expression d'un des interviewés et de fait conduire à des situations de troubles sociaux similaires à celles observées en Amérique Latine (Birdsall et al., 2014).

\section{Les politiques de transport: peu d'offres dédiées spécifiquement aux classes moyennes}

Actuellement, les politiques de transport ne répondent pas spécifiquement aux besoins des classes moyennes. Certains projets initiés sont davantage orientés vers les classes supérieures (entretien institutionnel $\mathrm{n}^{\circ} \mathrm{S} 3$ ) : infrastructures routières concentrées au centre d'Abidjan (3 $3^{\text {ème }}$ pont à péage, échangeurs VGE et Riviera 2, voies express dans la commune de Cocody); projet de bateaux climatisés dotés de wifi et de sièges individuels (via un partenariat public-privé avec les sociétés STL et CITRANS), au prix de 500 FCFA (prix supérieur à celui actuellement pratiqué pour les bateaux bus, et qui étaient déjà tournés vers une clientèle aux revenus élevés ) ; l'ouverture aux VTC (voitures de transport avec chauffeur), avec le développement de quatre entreprises privées dans ce domaine à Abidjan (Taxijet, Drive, EasyCab, Africab). La création des bus climatisés et dotés de wifi au tarif de 400FCFA par la SOTRA, les «Wibus », pourrait répondre aux besoins des strates supérieures des classes moyennes, bien que concentrés dans la commune de Cocody (entretien institutionnel $\mathrm{n}^{\circ} \mathrm{S} 4$ ). Certains projets, tel que le " métro » d'Abidjan ou bien la construction de routes (autoroute de Bassam, voie express Abobo-Anyama, pont de Jacqueville, etc.) profitent (ou profiteront) indirectement aux classes moyennes, bien que n'étant pas spécifiquement orientées vers elles. Mais l'essentiel des classes moyennes continuent pour l'heure d'utiliser les transports informels (gbakas, woro woro, éventuellement taxis individuels), dont les prix correspondent davantage à leurs revenus. L'enjeu de de cette politique est central dans une logique de ville soutenable à très haut niveau de classes moyennes. 


\subsubsection{Le secteur de l'énergie : de l'insatisfaction à la contestation}

Les politiques publiques du secteur de l'énergie illustrent parfaitement comment l'absence de réponse aux attentes des classes moyenne peut conduire à des débuts de contestation portée notamment par les strates hautes de cette catégorie.

\section{Du tout bydroélectrique à la libéralisation et à la diversification du secteur}

Des années 1950 aux années 1980, la Côte d'Ivoire choisit l'option de la production de l'énergie, via la production hydroélectrique, sous impulsion de l'Etat. En 1959, l'Etat colonial construit les barrages d'Ayamé 1 et 2. Félix Houphouët-Boigny réaffirme ce choix, par la construction, via la société d'Etat - la Société d'Energie Electrique de Côte d'Ivoire (EECI) - d'un ensemble de barrages à Kossou (1972), Taabo (1979), Buyo (1980), et Fayé (1983), afin d'assurer la demande nationale en énergie domestique et l'électrification des grandes villes ivoiriennes. Relativement rapidement, la Côte d'Ivoire engage une libéralisation et une diversification du secteur de l'énergie, et ce de manière pionnière pour la sous-région (OCDE, 2016a), poussé par la demande croissante en énergie et par le début de crise économique. En 1985, Félix Houphouët-Boigny ouvre le secteur aux entreprises privées ${ }^{30}$, afin qu'elles exploitent d'autres sources d'énergies (centrales thermiques, gaz naturel, pétrole brut, charbon). En 1990, sous la pression des Institutions de Brettons Woods, l'Etat procède à une restructuration du secteur énergétique en privatisant la structure d'État Énergie électrique de Côte d'Ivoire (EECI) au profit de la société privée, la Compagnie Ivoirienne d'Electricité (CIE, à l'époque essentiellement détenue par le groupe français Bouygues). Il lui accorde une concession relative au transport, à la distribution, à l'importation et à l'exportation de l'électricité (lui-même conservant la responsabilité du maintien des infrastructures et la fixation du prix) (Plane, 1997). La mise en production de champs de gaz naturel et pétroliers à partir de 1995 (le champ Panthère en 1995, puis Foxtrot en 1999), qui avaient été découverts dans les années 1970, ouvre l'ère de la production thermique de l'électricité à partir du gaz naturel. La Côte d'Ivoire accorde des contrats à des entreprises privées pour le financement, la construction et l'exploitation de centrales thermiques. Plusieurs centrales sont ainsi construites : en 1994, celle de Ciprel à Vridi (groupe français Eranove), en 1999 celle d'Azito (par un consortium international ${ }^{31}$ ), jusqu'à celles d'Agrekko 1 et 2 en 2010-2012 (groupe britannique). La production électrique repose ainsi aujourd'hui à près de $60 \%$ sur le thermique, à partir du gaz naturel, contre $40 \%$ via l'hydroélectrique.

\section{Les années Ouattara: d'une libéralisation accrue à la grogne sociale}

Lorsque Alassane Ouattara accède au pouvoir en 2010, les besoins en termes d'offre (amélioration de la qualité des infrastructures et puissance de production) et de demande (ménages et entreprises) sont forts. En 2012, seulement $56 \%$ de la population a accès à l'électricité, tandis que la Banque mondiale estime à $77 \%$ le taux moyen d'accès pour des pays se

\footnotetext{
$30 \quad$ Loi Nº5-583 du 29 Juillet 1985

31 Consortium d'Azito Energies constitué par IPS(WA), ABB, Electricité de France, et Globeleq.
} 
réclamant de l' "émergence ", se situant bien en-deçà des pays tels que le Brésil et le Viet Nam, qui présentent un taux d'accès proche de 100\% (figure $\mathrm{n}^{\circ} 5$ ). De la même manière, la consommation d'énergie électrique s'élève à $240 \mathrm{KWh}$ per capita, comparativement aux 500 estimés pour les pays émergents ${ }^{32}$. Le coût de l'électricité, bien qu'en deçà de la moyenne africaine, est fortement supérieur à celui pratiqué dans d'autres pays du Sud : en Côte d'Ivoire, il est estimé à environ 0,14 USD le kilowatt heure (2012), tandis qu'il se situe autour de 0,04 USD en Asie du Sud ${ }^{33}$.

En contre-point, les besoins en termes de production sont immenses : en 2014, la puissance électrique était de 1632 mégawatts (MW), tandis que les besoins estimés en 2020 par le Ministère de l'Energie et du pétrole seront proches du double (3000 MW). A l'instar des pays de la sousrégion, la Côte d'Ivoire fait face à plusieurs défis en matière énergétique, qui contribuent à maintenir un prix élevé : petitesse des réseaux, faiblesse du nombre d'entreprises industrielles (qui permettraient de faire des économies d'échelle), vieillissement des infrastructures, raccordements informels et problèmes de recouvrement des factures. Si la Côte d'Ivoire a l'avantage d'avoir développé un parc hydroélectrique de manière précoce - dont les coûts de production en énergie sont moins élevés que pour les hydrocarbures, elle est fortement défavorisée par les dix années de crise politique, qui ont coupé le pays en deux, avec des taux de recouvrements quasi-nuls dans les ex-zones Centre Nord et Ouest (CNO) entre 2002 et 2011.

Figure $n^{\circ} 5$ - Part de la population ayant accès à l'électricité (2012)

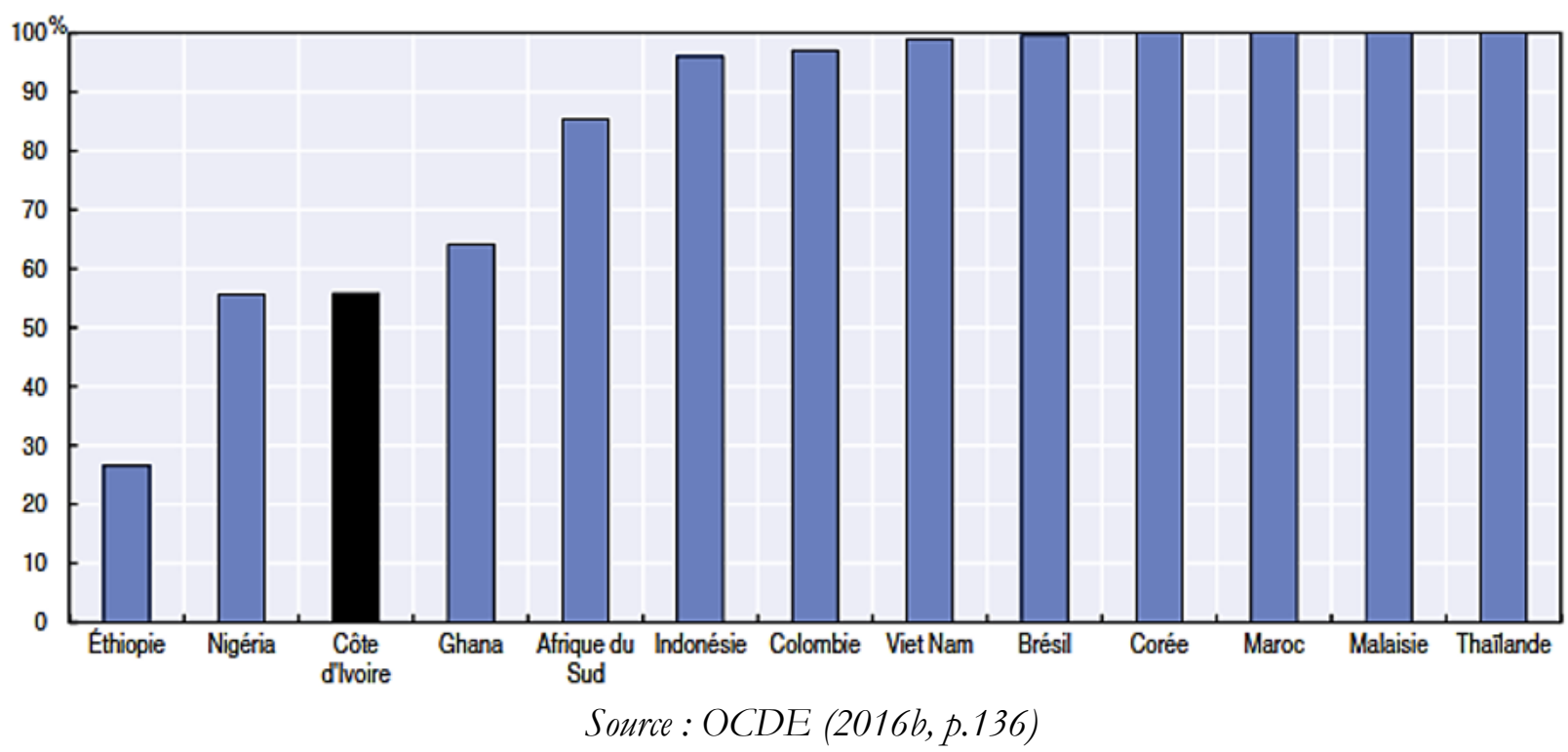

Ainsi, à partir de 2011, le nouveau gouvernement Ouattara se trouve face au défi d'investir massivement dans les infrastructures pour améliorer la qualité et la quantité de production énergétique, tout en veillant à ce que le prix ne se répercute pas sur les ménages. Il propose une

32 Banque mondiale, Indicateurs du développement dans le monde (base de données), Washington, DC, http://data.worldbank.org/products/wdi

33 http://www.jeuneafrique.com/239461/economie/electricite-pourquoi-les-africains-paient-ils-sicher/, page consultée le 13/11/2017 
stratégie à la fois développementaliste et libérale. Développementaliste, car il met en place plusieurs mesures à destination des plus vulnérables, tels que le Programme national d'électrification rural (PRONER) dont l'objectif est de raccorder les 1500 localités d'au moins 500 habitants à l'horizon 2015, et la totalité du pays en 2020 ; il promet la subvention (entre 30 à $75 \%$ ) des branchements au réseau pour les familles économiquement faibles. Il annonce également un plan de près de 400 milliards de Fcfa (610 millions d'euros) pour réaliser 1.200 kilomètres de ligne électrique et remettre en état tous les transformateurs. Libérale, car il choisit la voie de l'ouverture aux investisseurs étrangers, via l'introduction d'un nouveau Code de l'Electricité en $2014^{34}$. Ce Code a pour vocation d'autoriser des opérateurs privés à intervenir sur des activités anciennement dévolues à l'Etat, à savoir le transport, la distribution, la commercialisation, l'importation et l'exportation. Cependant, la place de l'Etat reste forte, conservant son monopole dans le maintien de l'offre et de la demande, et notamment sur la fixation des prix. De plus, le code de l'électricité prévoit que les prix doivent dorénavant tenir compte des coûts de production, des charges et des profits, mettant fin progressivement aux stratégies de tarification préférentielle, et promettant aux investisseurs une augmentation graduelle des prix. Ainsi, la Présidence Ouattara est marquée par une libéralisation accrue du secteur énergétique, avec notamment l'ouverture aux marchés Sud-Sud: construction du plus important barrage hydroélectrique à Soubré financé à 85\% par la Banque chinoise Exim Bank ou encore le projet de centrale électrique à Songon, financé par la compagnie publique chinoise China Energy Engeneering Corporation (CEEC).

Les mobilisations sociales qu'a connue la Côte d'Ivoire suite à l'augmentation du prix de l'électricité en 2015-2016 sont révélatrices des tensions inhérentes entre le choix d'approches développementaliste et libérale. Soutenu par la Banque mondiale, le gouvernement annonce le 20 mai 2015, l'augmentation du coût de l'électricité pour à la fois combler les déficits du secteur et rapprocher le prix de vente des couts de production. La mauvaise gestion de cette hausse, pouvant parfois aller jusqu'à $50 \%$, et sa répercussion immédiate sur les usagers sans en préciser les termes ont généré une forte grogne sociale et dans certains cas de véritables mouvements de révolte urbaines (notamment à Bouaké, Yamoussoukro, Daloa avec des attaques des agences de la CIE et d'autres infrastructures publiques et privées).

En parallèle de ces révoltes de rue, cette hausse du prix de l'électricité suscite un mouvement plus général au sein de la population, et ce notamment via les réseaux sociaux, c'est-à-dire une classe plus éduquée et ayant accès à internet, mais qui est directement touchée par les réformes du secteur de l'énergie, et dont une partie peut être associée à la classe moyenne et notamment à ses strates hautes. En avril 2016 est créé le «mouvement des 200 », via une page Facebook et le hashtag \#les200, porté notamment par Daouda Coulibaly, un journaliste et bloggeur indépendant. Ce mouvement est né suite à une déclaration malheureuse du Ministre de l'économie numérique, qui avait assuré lors d'une interview, que les revendications des internautes mécontents ne comptaient que 100 ou 200 personnes, car selon le Ministre, "seule une minorité pense que tout va mal en Côte d'Ivoire ». En réaction à cette affirmation soulignant la déconnexion de la classe dirigeante

$34 \quad$ Loi n 2014-132 du 24 mars 2014 
avec les réalités quotidiennes vécues par la majorité des ivoiriens, certains "cyberactivistes», tels qu'ils se définissent, créent « la page des 200 personnes qui pensent que le pouvoir d'achat des habitants de la côte d'ivoire devient de plus en plus faible » et qui estiment que la population ne doit pas payer le lourd tribut des dettes accumulées et investissements de l'Etat. Ils affichent des slogans tels que : «Le pays est devenu joli mais \#les200 ont le ventre vide » ou «\#les200 ne veulent plus du cosmétique. Ils veulent du concret ». Très vite, le mouvement dépasse le simple enjeu de l'électricité, qui est devenu le catalyseur d'autres revendications sociales, tels que la décision très contestée de l'opération de renouvellement systématique des permis de conduire (au prix de 10.000 FCFA), perçue comme une manœuvre politique au profit d'une entreprise proche du pouvoir ; ou bien la santé, le prix des matières premières, le chômage, la corruption, les enjeux fonciers, etc. Le hashtag \#les200 donne naissance à d'autres groupes affiliés, tels que \#grognesocialeCi225, l'« opération Abidjan ville noire $»^{35}$, ou l' « opération casserole », avec de nombreux témoignages qui affluent sur la toile.

Le secteur de l'énergie souligne les tensions et contradictions intrinsèques des politiques libérales du nouveau gouvernement, affichant des mesures en faveur des plus vulnérables tout en privilégiant, dans la pratique, l'assainissement du secteur et l'ouverture aux investisseurs étrangers. Cet épisode de grogne sociale souligne la déconnexion de la classe dirigeante avec la majorité des ivoiriens peinant à supporter une augmentation du prix de l'électricité. Il est également symptomatique d'une mobilisation sociale qui est initiée, non pas par des associations de la société civile spécialisées sur les enjeux de gouvernance et de contrôle citoyen, ni par des syndicats, mais par les consommateurs eux-mêmes, via la fédération des associations de consommateurs actifs ou bien les pages Facebook, dont le bien-être est directement amputé par ces mesures gouvernementales, et dont une grande partie représente la classe moyenne.

\subsubsection{La politique du logement et la promotion d'une classe moyenne de propriétaire : du discours à la pratique.}

"(...) aujourd'hui le Programme présidentiel de logement sociaux est exactement dédié à la classe moyenne, les opérations qui ont lieu à Songon et Bingerville sont des opérations à $60 \%$ social économique et $40 \%$ standing » (Entretien institutionnel $\mathrm{n}^{\circ} \mathrm{S} 8$ )

\section{Les évolutions des politiques du logement depuis les indépendances}

Après l'indépendance, pour faire face aux politiques d'urbanisation coloniales caractérisées par la construction de logements pour les élites locales et étrangères, via la SIHCI (société immobilière d'habitation de Côte d'Ivoire), Félix Houphouët Boigny initie une politique destinée à combler le manque d'habitat pour des classes moyenne et aisée alors en expansion. Lors du congrès du PDCI, en 1965, il déclare: "notre but est l'élimination de tous les taudis de Côte-d'Ivoire dans la prochaine décennie ». Cette annonce est suivie du lancement de la première politique publique d'habitat

35 L'« opération ville noire » a invité tous les ivoiriens à éteindre leurs lumières et appareils électriques le 1er juin 2017 , de $20 \mathrm{~h}$ à $20 \mathrm{~h} 30$ 
postindépendance en 1969, avec pour objectifs de résorber le déficit de logements, d'accélérer la promotion de l'urbanisation et de créer des logements de qualité. Il crée ainsi deux sociétés d'Etat, la Société immobilière de construction et de gestion immobilière (SICOGI) et la Société de gestion et de financement de l'habitat (SOGEFIHA). La SICOGI est chargée de réaliser des logements de tous types allant de la villa de standing ( 5 pièces) aux logements économiques (3 à 4 pièces). Les habitants peuvent obtenir de tels logements comme locataires ou propriétaires, ou sous forme de location-vente. Les habitats sont construits pour les populations aisées ou à revenus moyen. Aussi, le développement de l'habitat est allé de pair avec l'urbanisation, la politique nationale de rapprochement administration-administrés donnant naissance à de nombreuses villes à l'intérieur du pays (Bouaké, Daloa, Korhogo, Yamoussoukro, Man, Gagnoa, San-Pedro, Abengourou, Odienné, Bondoukou) qui, bien que plus petites que la métropole, ont contribué à entraîner des dynamiques régionales et économiques ainsi que la naissance de classes moyennes à l’intérieur du pays.

La crise économique des années 1980-1990 marque un coup d'arrêt dans les politiques du logement. Des difficultés croissantes dans l'accès au logement des populations apparaissent pour trois raisons. Premièrement, les salaires des individus diminuent, et notamment ceux des fonctionnaires, avec une baisse conséquente de leur pouvoir d'achat. Deuxièmement, la hausse du prix des matières premières liées aux matériaux de construction sur le marché national et international, la création de nouvelles taxations fiscales, conjuguée à la dévaluation du franc CFA qui interviendra plus tard (1993), engendrent une inflation sur les coûts des logements (location et vente). Enfin, l'Etat se désengage du secteur. En avril 1982, il cesse de payer des logements baillés et en 1983, il supprime les baux administratifs. Les crédits bancaires pour la construction immobilière sont de plus en plus rares et la suspension des prêts extérieurs provoque l'arrêt des programmes de construction des logements économiques. A partir de cette période, les investissements en matière de logement deviennent essentiellement le fait des opérateurs privés. Les crises politico-militaires des années 1990 et 2000, entrainent une stagnation du processus de développement urbain des villes ivoiriennes.

Les secteurs du logement et des transports ont vite constitué des secteurs critiques à partir des années 1960, l'accélération de la pression démographique et urbaine durant ces dernières décennies générant une crise structurelle du secteur du logement. Cette pression démographique est particulièrement critique sur l'offre de logements en Côte d'Ivoire. En 2013, le déficit cumulé en termes de logements était de 400.000, avec un accroissement de 10\% chaque année, répartis de manière équivalente entre Abidjan et l'intérieur du pays (20.000 par an pour chacun) ${ }^{36}$. Face à cette crise de l'offre immobilière, une première conséquence est l'augmentation continue du montant des loyers. Par exemple, pour un appartement de trois pièces, le coût s'élève à environ 300.000 FCFA à Cocody, un quartier résidentiel pour classes aisées, et entre $70.000 \mathrm{fcfa}$ et 120.000 FCFA pour son équivalent à Yopougon, un quartier plus populaire, quand le SMIG est fixé à 60.000 FCFA. En termes de perception des populations, on estime à $47 \%$ le taux de satisfaction des ivoiriens par rapport à l'offre de logement dans la ville ou la région qu'ils habitent

36 Selon le Bureau national d'études techniques et de développement 
(OCDE 2016a). Une deuxième conséquence est relative à l'augmentation des quartiers précaires à Abidjan, et ce particulièrement dans les quartiers populaires de Port-Bouet, Abobo et Yopougon, ainsi qu'à l'intérieur du pays.

\section{Le programme présidentiel de logements sociaux}

En 2010, la politique du logement est au cœur du programme d'Alassane Ouattara. Dans son programme, Mon projet pour la Côte d'Ivoire, afin de faciliter l'accès à la propriété privée il promet : "Accès à un logement, relance massive d'un habitat social réellement social, production en masse de terrains aménagés et de logements par des entreprises et promoteurs privés; mise à disposition des banques de ressources à taux zéro, mise en place de crédits acquéreurs à taux réduits sur 25 ans, mutualisation des risques pour "solvabiliser » les ménages ${ }^{37}$. En 2012, il lance ainsi le programme présidentiel de logements sociaux, en promettant une politique sociale en matière de logement ambitieuse: produire 60.000 logements, d'ici 2015. Pour son $2^{\text {ème }}$ mandat, et malgré les retards importants (3.533 logements sont alors en «cours de finition ${ }^{38}$ ), Alassane Ouattara fixe un nouvel objectif de 150.000 logements d'ici 2020. Ces logements doivent être répartis entre des logements économiques et sociaux $(60 \%)$ et de haut et moyen standing (40\%), sur 27 sites, dans les communes telles que Yopougon, Cocody (quartier Riviera, Angré Bessikoi, etc.) et dans les villes périphériques comme Bingerville, Bassam, Anyama, Songon, avec des projets de train urbains reliant ces différentes villes.

Pour ce faire, il ouvre le marché aux opérateurs privés (46 au total), avec une part importante dévolue aux entreprises internationales ${ }^{39}$. Si l'entreprise publique, la SICOGI, a pour objectif initial de contribuer à $25 \%$ de cet objectif global, des difficultés dans sa gouvernance interne (qui a conduit à la destitution de sa Directrice) la conduisent de facto, à être reléguée à l'arrière-plan, la quasi-totalité des logements construits étant finalement bâtis par des opérateurs privés (au moment de l'étude, en 2017). Cette politique de logements dite «sociale » à destination des "plus démunis », cible des individus avec un revenu inférieur à 400.000 FCFA, c'est-à-dire pour des individus qui se situent en haut des "classes moyennes » telles que définies dans cette étude. Cependant, les conditions d'accès sont restrictives pour ces «gens du milieu ». D'une part, la SICOGI cible directement les agents du secteur privé formel et les fonctionnaires, à l'exclusion du secteur informel rural et urbain, en raison de leur faible accès au système bancaire (SICOGI 2013). En effet, en Côte d'Ivoire, au-delà du faible taux de bancarisation de la population (15\%), les politiques de subvention et de soutien direct à l'acquisition de logement se sont avérés être des échecs (Badev et al, 2014). ). Les crédits immobiliers sont particulièrement faibles, représentant $2 \%$ du total des crédits alloués par le secteur bancaire, et bénéficiant à moins de 3\% des ivoiriens

37 Programme accessible à http://www.adosolutions.ci/ado/upload/828d2e458f41c4c4020adf389b76207c.pdf, page consultée le $13 / 11 / 2017$

38 Chiffres indiqués dans le programme d'Alassane Ouattara en 2015 : http://www.avecado.ci/logements-sociaux/

39 Dans le plan initial pour 2015, sur les 60.000 logements, 16.000 devaient être construits par la SICOGI, 4.000 par les constructeurs et promoteurs privés locaux, 20.000 par le groupe chinois C3R et 20.000 par le groupe égyptien El Mahmoudia (cf rapport SICOGI 2013) 
(OCDE, 2016b). De plus, les souscripteurs doivent garantir d'un apport initial de 10\% du prix total des logements, soit un montant d'environ 1,5 millions de FCFA (2.300 euros) minimum pour des logements dont le prix se situe aux environ de 15 millions de FCFA minimum (taxe comprise). La probabilité pour que ces individus «vulnérables » aient une épargne suffisante pour assurer ce montant est globalement faible. Selon les données de notre étude, le montant annuel moyen épargné par les ménages de la classe moyenne informelle en 2015 est de l'ordre de 300.000 FCFA. Autrement dit le montant de garantie demandé représente plus de 10 années d'épargne. Cette épargne étant essentiellement une épargne de précaution pour ces ménages, il est difficile d'imaginer qu'ils puissent parvenir à collecter un tel montant. Ainsi, ces politiques dites « sociales » ciblent en réalité les $20 \%$ les plus aisés des classes moyennes. D’autre part, le prix des logements est prohibitif pour des personnes dites "économiquement faibles ». L'Etat a fixé par ordonnance $^{40}$ un prix unitaire maximum de vente hors taxe de 12,5 millions de FCFA (environ 19.000 euros) pour les logements à caractère social et de 23 millions de FCFA (environ 35.000 euros) pour les logements à caractère économique. Dans la pratique, les opérateurs économiques appliquent des prix plus élevés. Ils dénoncent le manque de respect des garanties de l'Etat (non viabilisation des terrains, absence de fond de garantie auprès des banques et taux d'intérêts promis à 5,5\% non respectés) ainsi que tous les frais annexes à leurs charges (travaux de voierie, réseau et drainage, caniveaux et adduction d'eau, éclairage, coûts des hypothèques, frais de notaires, études, etc. $)^{41}$. Cette libéralisation du marché conduit les opérateurs à fixer leurs propres prix de vente et à choisir leurs clients. Ces politiques du logement dites «sociales» bénéficient, au final, aux individus économiquement les plus solvables, et consolident une redistribution de la rente via les réseaux politico-économiques (via l'usage de prête-noms), au détriment de flux entre classes sociales. Si les «gens du milieu » ivoiriens sont exclus de ce projet social d'accès à la propriété, les gens d'« en bas » résidant dans des quartiers précaires, voire des bidonvilles, sont sujets à de vastes campagnes de déguerpissements (Bouquet et Kassi-Djodjo, 2014), sans proposition de relogement. Ainsi, en dépit de l'annonce d'une politique «développementaliste » en matière de logements, où l'Etat reprendrait une place de facilitateur entre opérateurs privés et individus vulnérables, cette politique a glissé, dans la pratique, dans une logique libérale, sans réel contrôle ni régulation efficace de la part de l'Etat.

\subsubsection{La santé et la couverture maladie universelle (CMU): quelle protection des classes moyennes vulnérables?}

En Côte d'Ivoire, de la période postindépendance à nos jours, l'évolution de l'accès à la santé, se caractérise par la permanence d'un dispositif de protection sociale inégalitaire, couvrant uniquement les acteurs du secteur formel (public et privé) et par une privatisation progressive de l'offre de santé, au détriment des plus vulnérables.

\footnotetext{
$40 \quad$ Ordonnance n² 2017-279 du 10 mai 2017

41 Sources : entretiens institutionnels et http://news.abidjan.net/h/616040.html
} 


\section{De la gratuité des soins aux PAS et à l'Initiative de Bamako}

Durant la période postindépendance (1960-1980), Félix Houphouët-Boigny adopte la gratuité des soins pour toute la population. A l'époque, l'offre de santé héritée de la colonisation est essentiellement publique, soutenue par certains établissements de santé privés confessionnels, avec une offre privée minime. Ce système est facilité par le contexte économique et politique, qui permet de dégager les ressources nécessaires pour le financer (Oga et al, 2011). Cependant, la crise des années 1980 génère une interruption de cet équilibre. Les plans d'ajustement structurels (PAS) contraignent l'Etat à diminuer la part du budget alloué à la santé : de 10,6\% en 1970, celleci diminue à 7\% dans les années 1985-1988, bien que la baisse ait été amorcée avant la mise en œuvre des PAS (Brunet-Jailly, 1992). Plusieurs mesures pour libéraliser le secteur de la santé sont initiée : limitation du nombre de médecins à l'entrée de la Faculté de médecine, aide aux médecins qui souhaiteraient créer leur cabinet ou clinique privée et adoption du recouvrement des coûts (promu dans l'Initiative de Bamako en 1987), présentée comme une "pièce maîtresse du redressement du système sanitaire ». Ce plan promulgue un taux de recouvrement de 80\% dans les CHU (en 1992, part qui devra atteindre $60 \%$ en 1994) et de $40 \%$ dans les formations primaires. Ainsi, la gratuité des soins est remplacée par un système inégalitaire, dans la mesure où les populations les plus défavorisées se rendent davantage dans les centres de santé primaire. Promouvant la privatisation, ces orientations occultent les défis majeurs telle que la détérioration dramatique de la qualité des soins dans les structures publiques, alors amorcée par une baisse des investissements privés liés à la crise économique (Brunet-Jailly, 1992). Les individus des classes moyennes du secteur formel bénéficient quant à eux de certains filets de sécurité. Les fonctionnaires sont couverts par la Mutuelle Générale des Fonctionnaires et Agents de l'Etat (MUGEFCI) depuis 1973. Les employés du secteur privé formel (essentiellement les grandes entreprises) peuvent avoir accès à des soins au sein des centres médicaux ou sanitaires de leurs entreprises (rendus obligatoires depuis 1977 pour les entreprises de plus de 100 salariés), à des remboursements de frais de santé liés aux accidents du travail et maladies professionnelles (via la CNPS créé en 1968 ${ }^{42}$ ), ainsi qu'à des mutuelles ou assurances maladies d'entreprises ou des soutiens ponctuels et directs de leurs employeurs. Pour les agriculteurs, des mutuelles rurales, créées sur la loi des associations (1960) se créent, offrant une couverture maladie, mais qui reste généralement insuffisante (faible taux de recouvrement des cotisations, absence de priorité de la santé).

Depuis la fin des années 1980, le système de santé s'est largement dégradé, présentant des indicateurs nationaux au plus bas. En 2015, avec une espérance de vie de 53 ans, la Côte d'Ivoire est comparable à la République centrafricaine et se situe loin derrière d'autres pays se réclamant de l'émergence tels que, dans la sous-région, le Sénégal (69ans) ou ailleurs le Brésil, la Turquie et le Viet Nam (autour de 79-81 ans) (cf. figure $n^{\circ}$ 6).

42 La CNPS a remplacé le régime des risques professionnels créé en 19658, afin de prévenir et de réparer les accidents du travail et les maladies professionnelles par l'action de soins médicaux, d'indemnités journalières et de rentes 
Figure $n^{\circ} 6$ - Espérance de vie en Côte d'Ivoire

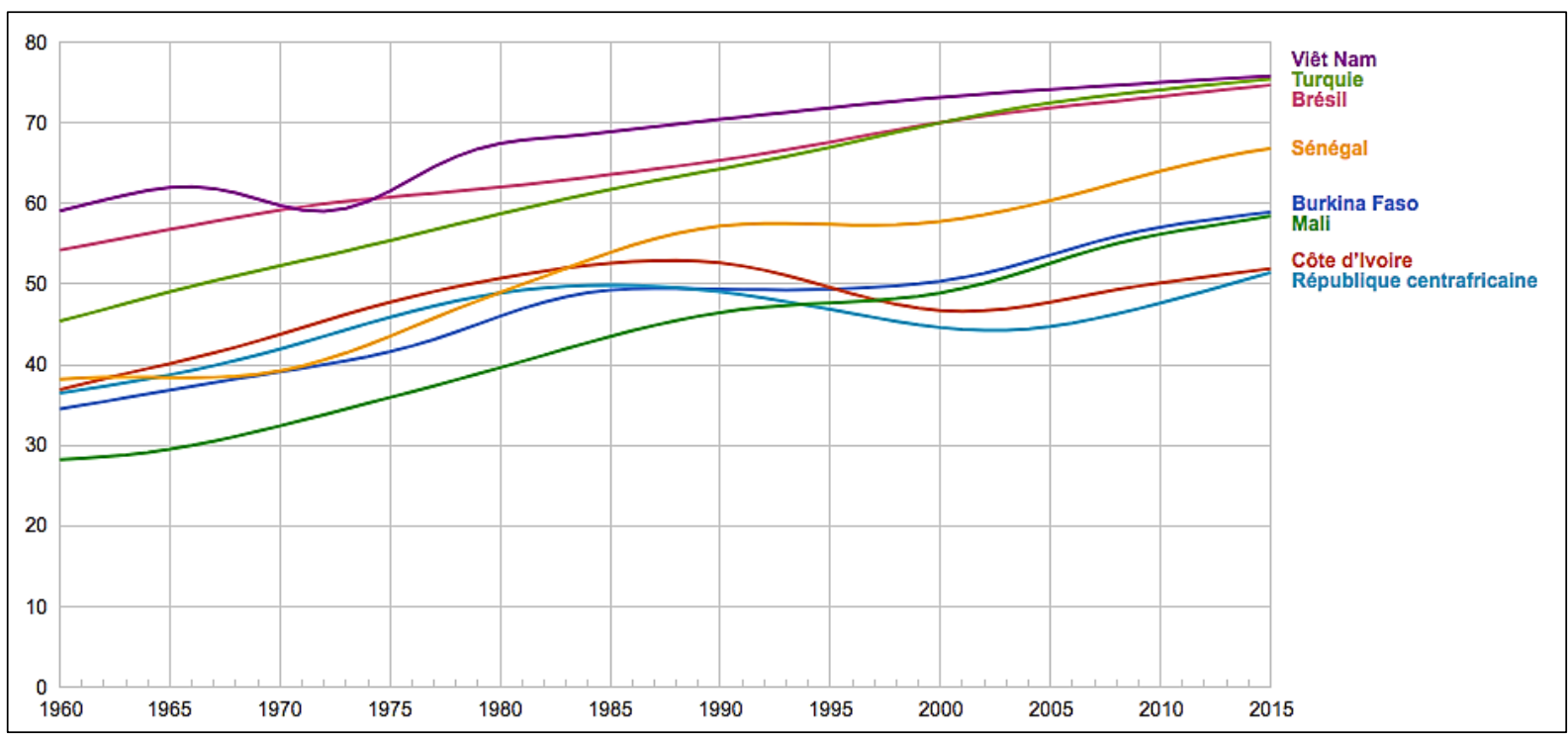

Source : World Development Indicators

Une cassure s'est opérée à partir de 1985, en raison de la crise économique et des PAS d'abord, puis de la succession de crises politiques. La coupure du pays en deux à partir de 2002 a été particulièrement dommageable pour le système de santé national, avec un exode massif du personnel de santé exerçant dans les zones Centre Nord et Ouest ${ }^{43}$, une absence de maintenance et d'investissement dans les infrastructures de santé, et des pénuries en médicaments et intrants. L'offre de santé publique est peu attractive, avec un taux d'utilisation des services par la population estimé à 18\% (MSLS/CPS 2008), tandis que les structures de santé privées se sont fortement développées depuis les années 1990 avec, en terme absolu, un nombre équivalent de structures de santé publique (2067 - réparties sur les niveaux de la pyramide sanitaire) et privées (2085 - polycliniques, cliniques, centres et cabinets médicaux, infirmeries privées, officines privées, centres confessionnels) en 2011 (MSHP, 2016). Si le nombre des structures de santé privées a explosé depuis les années 1990, leur contrôle par l'état reste défaillant, l'association des cliniques privées de Côte d'Ivoire estimant à $70 \%$ le nombre de structures de santé privées illégales en $2016^{44}$.

L'accessibilité à l'offre de santé est inégalitaire, tant d'un point de vue géographique (la capitale économique concentrant l'essentiel de l'offre de santé), qu'économique. La Côte d'Ivoire connaît un taux de pénétration des systèmes de prépaiement et de couverture du risque maladie particulièrement faible (4\%), couvrant la tranche supérieure des classes moyennes et classes supérieures, via la mutuelle des fonctionnaires, la MUGEFCI $(1,8 \%)$, les mutuelles de corporations publiques $(1,2 \%)$, la CNPS $(0,5 \%)$, les entreprises privées $(0,2 \%)$ et les compagnies d'assurances privées (0,1\%) (2008) (MSLS 2012). Ainsi, 96\% des dépenses faites par les ménages sont directement issues de leurs ressources propres (ENV, 2008). Le secteur de l'assurance privée est encore relativement embryonnaire - bien qu'en expansion, étant donné son prix prohibitif,

43 Au 31 mai 2003, la DRH de la santé enregistrait à Abidjan, 2417 agents déplacés soit 69\% des 3.500 personnels du secteur public en service initialement dans les zones CNO, (cf. MSHP 2008))

44 Voir, http://news.abidjan.net/h/597615.html, page consultée le 13/11/2017 
tout en étant généralement subordonné à d'autres contrats d'assurance (accidents, incendies, etc.), et réservée en grande majorité aux salariés des entreprises privées ${ }^{45}$. En dehors des salariés du secteur privé formel, les strates basses de la classe moyenne n’ont pas les moyens de souscrire à ce type de contrats.

\section{Les tentatives de couvertures maladies universelles : de l'AMU à la CMU}

Afin de remédier à l'inaccessibilité des soins aux plus démunis, Laurent Gbagbo avait instauré un système ambitieux d'assurance maladie universelle (AMU) en 2001, sous fond d'idéologie socialiste. Fondé sur la solidarité nationale et sur son caractère obligatoire, il astreignait chaque bénéficiaire à une contribution financière sous la forme d'une cotisation en fonction de sa capacité contributive et s'appliquait à tous les résidents de Côte d'Ivoire, ainsi qu'aux ivoiriens de l'étranger. Il fournissait un paquet minimum de prestations (PMA, tel que défini depuis décembre 1996) avec un ticket modérateur à la charge des bénéficiaires variant entre $0 \%$ (niveau primaire de la pyramide sanitaire) et 20-30\% (niveaux secondaire et tertiaire). Or, faute de ressources pour sa mise en œuvre, ce système n'a pas pu voir le jour : les décrets d'application n'ont pas été tous adoptés (5 sur 30 en 2006) et ont laissé de larges marges d'imprécisions (chevauchement avec d'autres systèmes antérieurs telles que la CNPS et la MUGEFCI, articulation avec les régimes complémentaires, etc.). En 2014, le projet de Couverture maladie universelle (CMU) que le Président Alassane Ouattara avait promis lors de sa campagne électorale, est voté à l'unanimité. Fondée sur les mêmes principes que l'AMU (universel, obligatoire, contribution variable selon les capacités), cette nouvelle couverture se veut en être une version simplifiée. Deux régimes sont proposés : un contributif avec une cotisation de 1000 FCFA par mois et par personne (à partir des enfants de plus de 5ans) et un autre non contributif et subventionné par l'Etat pour les personnes en situation d'indigence (critères de cette catégorie encore non définis), donnant accès à un paquet minimum d'activités et au remboursement compris entre $70 \%$ et $80 \%$ dans les centres de santé conventionnés. Alors que l'AMU prévoyait la création de trois organisations ad hoc (la Caisse sociale agricole pour le secteur agricole, la Caisse nationale d'assurance-maladie pour tous les autres secteurs et le Fond national d'AMU chargé de chapeauter ces deux caisses), la CMU est constituée d'une seule caisse, la Caisse Nationale d'Assurance Maladie (CNAM). Ce projet est présenté comme étant la politique sociale phare du gouvernement Ouattara. Depuis son lancement en 2015, les campagnes d'enrôlement dans tout le pays bénéficient d'une large couverture médiatique ${ }^{46}$, sans que la mise en œuvre effective du programme ait encore eu lieu. La phase pilote conduite auprès des étudiants est en effet encore récente (et ne permet pas de rendre compte de son effectivité au moment de l'étude), et les promesses de financements de l'Etat (promesse de 60 milliards de FCFA en 2016 pour les plus vulnérables) se font attendre. Afin de préparer la forte affluence des populations dans les structures de santé, l'Etat a entrepris un

45 Une étude de Gras Savoye estimait que $80 \%$ de leurs clients sont des entreprises et $20 \%$ des personnes physiques (cf Lezou 2006)

46 Par exemple, la plateforme de journaux ivoiriens «Abidjan.net» consacre un dossier spécial à la CMU, avec près de 200 articles consacrés à ce sujet, et au lancement dans tout le pays des opérations d'enrôlement. https://news.abidjan.net/dossiers/articles.asp?id=2313 (consultée le 9 novembre 2017) 
chantier de réhabilitation des établissements, d'abord de $2^{\text {ème }}$ niveau (Centres hospitaliers régionaux et Hôpitaux généraux), puis de $3^{\text {ème }}$ niveau, via un partenariat public privé, qui débutera en 2018, ce qui a encore retardé le lancement effectif de la CMU. Les critères définissant la catégorie des « indigents » n'ont pas encore été définis : dans un pays où $67 \%$ de la population vit avec moins de $4 \$$ par jour, l'Etat fait face à l'épineuse question de soutenir le segment le plus pauvre de la population, tout en demeurant économiquement viable ${ }^{47}$. Dans ce contexte, les "floating class» et classes moyennes inférieures, c'est-à-dire la tranche la plus nombreuses de la population, risquent de se trouver directement impactées par l'obligation de payer une contribution de 1.000 FCFA par personne, ce qui représente une forte dépense pour des ménages aux revenus compris entre 15.000 FCFA et 30.000 FCFA voire 40.000 FCFA. La réception de ce projet oscille entre espoir et scepticisme. Depuis trois ans, aucun changement effectif n'a été constaté par la population, qui reste marquée par l'ineffectivité de l'AMU, dans un contexte de défiance vis-à-vis du pouvoir en place. De plus l'attribution du marché de l'enrôlement biométrique des futurs souscripteurs à la CMU a été confiée, via un partenariat public privé, au groupe SNEDAI, détenu par le puissant et critiqué Adama Bictogo, déjà bénéficiaire de nombreux contrats avec l'état. Ce dernier cristallise une certaine méfiance, exprimée notamment par Mr. H (entretien ménage $\mathrm{n}^{\circ} 27$ ), tant son positionnement semble refléter la concentration actuelle des pouvoirs via l'enchevêtrement d'activités politiques et économiques.

En dehors de la CMU, le gouvernement Ouattara a initié différentes stratégies d'exemptions de paiement des frais de santé, sans cibler de manière particulière les revenus des ménages. En avril 2011, au sortir de la crise électorale, l'Etat déclare l'exemption totale des frais de prise en charge médicale des usagers dans les établissements sanitaires publics, parapublics et communautaires conventionnés (excluant le privé), qui durera 9 mois et génèrera une désorganisation importante du système de santé (engorgement des structures publiques - et notamment des centres de santé du $3^{\text {ème }}$ niveau, rupture d'intrants et médicaments essentiels, arriérés de paiement du personnel médical, etc.). Une exemption ciblée et sélective a ensuite été adoptée en 2012, auprès des femmes enceintes, des enfants de moins de 5ans, et pour le paludisme. Ainsi, au vu de cette perturbation du secteur public, il a été observé que les catégories aisées ou classes moyennes supérieures, peuvent se tourner vers le privé, tandis que les pauvres sont contraints à rester sans soins (Oga et al., 2011).

Par ailleurs, les politiques publiques en matière de santé se caractérisent également par leur forte extraversion c'est-à-dire qu'elles sont essentiellement financées et façonnées par des bailleurs (publics et privés). La part des dépenses de l'Etat dans le secteur de la santé (5,7\% du PIB) a connu une chute depuis la fin des années 1990 et reste bien en-deçà des objectifs de la déclaration d'Abuja (15\% du PIB). De plus, la Côte d'Ivoire connaît le paradoxe d'être l'un des pays de la sous-région ouest africaine avec le taux le plus élevé de dépense totale de santé par rapport à son PIB, mais avec l'un des taux les plus faibles des dépenses publiques dans la dépense totale de santé (laissant une grande part au secteur privé - essentiellement les ménages).

47 Au moment de l'enquête, la définition des «indigents» était confiée à l'Institut national de la statistique (INS). 
C'est ainsi que la gratuité du dépistage et du traitement pour des pathologies comme le VIH/sida, la tuberculose et le paludisme a pu être adoptée, étant fortement financées, notamment par le Fonds mondial de lutte contre le VIH/sida, la tuberculose et le paludisme et le Programme d'urgence présidentiel américain, le Pepfar. D'autres pathologies, telles que le cancer et les hépatites virales sont essentiellement soutenues par des partenariats avec des compagnies pharmaceutiques (des programme "Access Roche») qui offrent certaines réductions des coûts, mais laissent à la charge du patient le complément (dépistage, frais chirurgicaux, médicaments complémentaires, etc.), ce qui engendre dans la pratique un coût prohibitif pour la grande majorité des ivoiriens qui n'ont pas d'assurance maladie ${ }^{48}$. Encore, selon le budget de l'Etat de $2017^{49}$, la lutte contre Ebola a obtenu un budget de 21 millions $€$, sans un seul cas, contre des budgets compris entre $100.000 €$ et $150.000 €$ par an pour les programmes nationaux de lutte contre le diabète, le cancer, le tabagisme, la santé bucco-dentaire, ou les hépatites, qui constituent de véritables enjeux de santé publique ${ }^{50}$. Ainsi, hormis les politiques de lutte contre le VIH, le paludisme et la tuberculose, qui bénéficient effectivement à l'ensemble de la population, toutes classes confondues, mais qui demeurent fragiles car essentiellement dépendantes des dons internationaux, il n'existe pas de politique de santé publique qui permettrait de soutenir les individus - à l'exception des plus riches - des différentes pathologies qui touchent ivoiriens.

Les résultats de ce troisième chapitre montrent à quel point il est difficile d'identifier des politiques publiques dédiées explicitement au public des «classes moyennes » à la fois en raison de la forte hétérogénéité de cet ensemble, des arbitrages que doivent réaliser les dirigeants d'un pays en développement comme la Côte d'Ivoire, entre lutte contre la pauvreté et efficacité économique des politiques mises en œuvre, et des pressions politiques concurrentes qui s'exercent dans la fabrication de ces politiques. Ainsi, certaines politiques présentées explicitement comme ciblant les classes moyennes ivoiriennes ne visent en réalité que les catégories les plus aisées de la population où alors les strates hautes de la classe moyenne comme dans le cas de la politique de logement social. Le ciblage « classe moyenne » permet au pouvoir de se légitimer sans mettre en péril ses capacités budgétaires. Inversement, des politiques publiques qui ne sont pas expressément dédiées aux classes moyennes sont susceptibles de les affecter. D’une part, des politiques sectorielles qui ne ciblent pas directement des catégories sociales spécifiques ont des effets directs sur leurs capacités. Les politiques industrielles, agricoles, commerciales et de l'emploi lancées par le président Ouattara affectent l'environnement dans lequel agissent les classes moyennes. L'Etat ivoirien fixe des règles et des orientations et laisse les groupes sociaux agir et saisir les opportunités offertes par cet environnement dans lequel le

48 Par exemple, pour le cancer du sein, la mammographie reste à la charge du patient, et se fait généralement tardivement, une fois que le cancer est avancé, avec un appareil opérationnel uniquement disponible au CHU de Treichville. Les traitements sont donc inefficaces, tandis que les opérations chirurgicales restent à la charge du patient.

49 Voir le Budget de la République de Côte d'Ivoire 2017, disponible à

http://www.caidp.ci/uploads/3263d61d8591b4e8bafd27f3d5b7dbca.pdf, page consultée le $13 / 11 / 2017$

50 La prévalence du diabète est estimée à 5\% selon la Fédération international du diabète (FID) (2015), la prévalence des hépatites $\mathrm{B}$ à $12 \%$ et des hépatites C à $5 \%$ (données CNTS), tandis que l'on estime à 12.000 nouveaux cas de cancer par an (GLOBCAN 2012, http://globocan.iarc.fr/Pages/fact sheets population.aspx) 
secteur privé se positionne très largement. D'autre part, des politiques comme celles de la santé et de la couverture maladie, qui semblent se focaliser spécifiquement sur les populations les plus pauvres et vulnérables, vont inversement profiter en priorité à certains groupes de classes moyennes qui pourront disposer de services améliorés.

\section{Conclusion}

En conclusion, après cette analyse approfondie de l'essor des classes moyennes et de l'impact de celui-ci sur les politiques publiques en Côte d'Ivoire, nous pouvons tirer les enseignements principaux suivants :

- Comprendre le thème de l'émergence des classes moyennes et ses enjeux contemporains en Côte d'Ivoire impose de revenir sur le temps long du régime de croissance, des équilibres politiques et des processus de stratifications sociales. Près de trente années d'instabilités économiques, financières et politiques ont particulièrement malmené les «classes moyennes » émergentes issues du «miracle ivoirien» de l'après indépendance. Alors que le pays renoue avec un cycle de forte croissance économique depuis 2011, les ambitions d'une Côte d'Ivoire émergente à l'horizon 2025 supposent l'accroissement et la consolidation de ces catégories intermédiaires de revenus aux caractéristiques fortement marquées par l'histoire économique ivoirienne.

- Aujourd'hui en Côte d'Ivoire, l'ampleur de la classe moyenne de revenu telle que nous l'identifions (4\$ - P95) est limitée à $26 \%$ de la population, cela reste néanmoins assez significatif au regard des standards de la région. Dans cet ensemble on peut identifier des traits communs : un goût partagé pour l'effort sur soi et pour la promotion de soi; une même importance accordée à l'éducation des enfants ; un même effort soutenu d'épargne ; un même positionnement très individualiste et matérialiste, une identification commune aux « ni riches ni pauvres ».

- Cette classe moyenne ivoirienne est particulièrement hétérogène en termes de catégorie socioprofessionnelle, d'éducation ou de statut dans l'emploi. Nos résultats soulignent qu'elle est constituée de cinq groupes distincts : (i) les agriculteurs, (ii) les travailleurs de l'économie informelle, (iii) la classe moyenne "intermédiaire" du secteur privé formel, (iv) les dirigeants, cadres et professions intermédiaires du secteur public et (v) les retraités et inactifs.

- Au-delà de ces cinq groupes un fort dualisme se dégage en termes de revenu et d'informalité des positions socioprofessionnelles. D'un côté, on retrouve en minorité (21\%) une strate haute et stabilisée de la classe moyenne constituée plutôt d'héritiers des groupes intermédiaires des années 1960-1970 (salariés du secteur formel privé et public). De l'autre, on observe une strate basse majoritaire (79\%) composée de ménages en situation de petite prospérité et de grande vulnérabilité. Ces néo-classes moyennes associent les indépendants informels urbains et les agriculteurs. 
- En Côte d'Ivoire, pas plus que dans la quasi-totalité des pays africains, la notion de classe moyenne ne peut prendre un sens sociologique et politique articulé autour de l'idée d'une conscience d'appartenance collective. Il n'en demeure pour autant pas moins que nos entretiens auprès des ménages ont mis en lumière un certain nombre d'attentes et d'aspirations au regard de plusieurs secteurs de politiques publiques. L'éducation apparaît comme le secteur qui cristallise le plus d'attentes chez les classes moyennes, ces dernières dénonçant unanimement les errements du système public d'éducation et l'absence de politiques tournées vers l'avenir. Ces classes moyennes sont à la recherche de meilleurs revenus, de plus de stabilité et de confort d'habitation. Elles dénoncent de manière quasi générale le creusement des inégalités sociales et la corruption.

- Nos résultats ont montré que si certaines politiques publiques sont présentées explicitement comme ciblant les classes moyennes ivoiriennes, elles touchent en réalité que les catégories les plus aisées de la population et les strates hautes de la classe moyenne (politique de logement social par exemple). Inversement, des politiques publiques qui ne sont pas expressément dédiées aux classes moyennes sont susceptibles de les affecter (les politiques industrielles, agricoles, commerciales et de l'emploi).

- Les classes moyennes ivoiriennes sont très peu engagées politiquement. Elles peuvent d'autant moins s'exprimer que leur forte hétérogénéité interne et leur faible expérience de mobilisation collective réduisent fortement leur capacité de faire pression sur le politique, sauf pour des groupes qui interviennent alors plus sur la base de leur identité socio professionnelle (fonctionnaires et salariés du secteur privé formel) que de leur appartenance à la classe moyenne. Pour le moment dans le cas ivoirien, le politique oriente et contraint donc peu les comportements et choix de ces classes moyennes, que l'on laisse assez volontiers saisir les opportunités qui s'offrent à elles sur le marché. Mais qu'en sera-t-il, par exemple, lorsque face à des groupes de classe moyenne stabilisés, plus nombreux et unifiés, le gouvernement devra dans une logique de ville durable imposer des règles d'urbanisme vertical, des restrictions à l'accès à l'automobile... ? 


\section{Bibliographie}

Acemoglu, D., Johnson, S. and Robinson, J.A. (2001), "The Colonial Origins of Comparative Development: An Empirical Investigation," American Economic Review, Vol. 91 (December), p. 1369-1401

AEO (2017), Côte d'Ivoire, Rapport sur les Perspectives Economiques en Afrique, BAD-OCDE-PNUD.

Affou Yapi, S. (1987), Les planteurs absentéistes de Côte d'Ivoire, Editions de l'ORSTOM, Collection travaux et documents $n^{\circ} 210$, Paris.

African Development Bank, (2011), "Dynamics of the Middle Class in Africa", Market Brief, April 20, 2011

AGEPE (2014), «Rapport sur l'emploi et la formation en 2012 », Agence d'Études et de Promotion de l'Emploi de Côte d'Ivoire.

AGEPE (2013), «Situation de l'emploi en Côte d'Ivoire en 2012 », Rapport de synthèse, Agence d'études et de promotion de l'emploi (Agepe), Abidjan, http://www.agepe.ci/etudespublications/179/situation-de-l-emploi-en-ca-te-d-ivoire-en-2012.

Akinkugbe, O. and Wohlmuth, K. (2016), Africa's middle class, Africa's entrepreneurs and the 'missing middle', in Melber, H. (ed), The Rise of Africa's Middle Class, ZedBooks, London, pp.69-94

Akindès, F. (2002), Inégalités sociales et régulation politique en Côte d'Ivoire. La paupérisation en Côte d'Ivoire est-elle réversible ?, Politique africaine 2000/2, 78, p. 126-141.

Amin, S. (1967), Le développement du capitalisme en Côte d'Ivoire, Editions de Minuit, Paris, 520 p.

Badev, A., Beck, T., Vado, L. et Walley, S. (2014). "Housing finance across countries, new data and analysis", Policy Research Working Paper, Banque Mondiale, Washington, DC.

Balac, R. (2016), Stratégies des jeunes dans une économie de plantation de l'Ouest ivoirien. Étude d'un terroir, Afrique contemporaine, 2016/3 (N²59), p. 41-58.

Bamba, A.B. (2016), African Miracle, African Mirage. Transnational Politics and the Paradox of Modernization in Ivory Coast, OHIO University Press, Athens, Ohio.

Banerjee, A., Duflo, E. (2008), "What is middle class about the middle classes around the world?”, Journal of Economic Perspectives, 22(2): 3-28.

Banque Mondiale (2015a), «Performances Économiques Ivoiriennes depuis la Fin de la Crise Post-Électorale », Rapport sur la situation Économique récente en côte d’ivoire, n 95601 , World Bank, Washington. 
Banque Mondiale (2015b), «La Force de l'Eléphant. Pour que sa croissance génère plus d'emploi de qualité ». Rapport Situation Economique Côte d'Ivoire, Décembre 2015.

Banque Mondiale (2017), «Le défi des compétences. Pourquoi la Côte d'Ivoire doit réformer son système éducatif ? ", Rapport Situation Economique en Côte d'Ivoire, 4ème édition, Banque Mondiale.

Birdsall, N., Lustig, N., and Meyer, C. J. (2014). The Strugglers: The New Poor in Latin America? World Development, 60, 132-146.

Berg, A., J. Ostry and J. Zettelmeyer (2012), What Makes Growth Sustained?, Journal of Development Economics, 98, pp. 149-166.

Berrou, J.-P. (2014), Les entrepreneurs du secteur informel, in: M. Grossetti, P.-P. Zalio et P.-M. Chauvin (eds) Dictionnaire sociologique de l'entrepreneuriat, Presses de Sciences Po, Paris.

Berrou, J.-P. et Eekhout, T. (2018), L'économie informelle : un défi au rêve d'émergence de l'Afrique ?, in La sécurité africaine à l'heure de l'émergence: nouveaux enjeux pour les Relations Internationales, Durand de Sanctis, J. et Abourabi, Y. (eds), Karthala, à paraitre.

Berrou, J.-P., Combarnous, F. et Eekhout, T. (2017), «Les TIC : une réponse au défi du développement des micro et petites entreprises informelles en Afrique sub-saharienne ? ", LAMORANGE. http://recherche.orange.com/wpcontent/uploads/2017/02/2017 01 LAM ORANGE TICInformel EtatDeLArt.pdf

Berrou, J.-P. et Mellet, K. (2017), "Les petites entreprises informelles africaines au défi des technologies numériques ", Blog de la Recherche - Orange, https://recherche.orange.com/lespetites-entreprises-informelles-africaines-au-defi-des-technologies-numeriques /

Berthélemy, J.-C. and F. Bourguignon (1996), Growth and crisis in Cote d'Ivoire. Washington, D.C., World Bank, 240 p.

Bonnefond, C., Clément, M., Combarnous, F. (2015), "In search of the elusive Chinese urban middle class: an exploratory analysis“, Post-Communist Economies, 27:1, pp.41-59.

Bossuroy, T. and Cogneau, D. (2013), Social Mobility in Five African Countries, The Review of Income and Wealth, 59 (Special Issue), pp.84-110.

Bouquet, C. (2011), Côte d'Ivoire. Le désespoir de Kourouma, Armand Colin, $3^{\text {ème }}$ Edition, Paris

Bouquet, C. et Kassi-Djodjo, I. (2014), « « Déguerpir » pour reconquérir l'espace public à Abidjan. », L'Espace Politique, 22 (1).

Boussou, M. (2017), De l'efficacité des mots et concepts dans la définition des politiques économiques : étude du cas de la Côte d'Ivoire à travers une analyse des discours, Thèse de Doctorat, Université Paris-Saclay. 
Brunet-Jailly J. (1992), «L'ajustement macro-économique et le secteur de la santé : deux exemples africains, le Mali et la Côte d'Ivoire », in Crises et ajustements en Côte d'Ivoire, les dimensions sociales et culturelles, Actes de la Tables Ronde de Bingerville (30 novembre - 2 décembre 1992), p. $27-47$

Brunke, B., Van Dongen, B., \& Downey, W. (2013), “Global topics 8 billion consumers - How to reach emerging market consumers with new strategies". Munich, Germany: Roland Berger Strategy Consultants.

CAPEC (2014), «Étude sur l'effet de la croissance économique en Côte d'Ivoire sur les conditions de vie des populations, mars $2014 »$.

CFAO (2015), «Les classes moyennes en Afrique, quelle réalité ? quels enjeux ?», White Paper, CFAO-IPSOS-Bearing Point.

Chauveau, J.-P. et Dozon, J.-P. (1988), Ethnies et État en Côte-d'Ivoire, Revue française de science politique, 38 (5), pp. $732-747$.

Cogneau, D. (2007), L'Afrique des inégalités : Où conduit l'histoire, Collection CEPREMAP, Editions rue d'ULM.

Cogneau, D. and Czajka, L. (2016), "Inequalities in Africa: Measurement and Patterns », International Workshop on Inequality and Middle Class Development in Africa, GIGA, Cape Town, 05-06 May 2016

Cogneau, D. et Mesplé-Somps (2002), «L'économie ivoirienne, la fin du mirage ? », DIAL, DT/2002/18.

Cogneau, D., Bossuroy, T., De Vreyer, P., Guenard, C., Hiller, V., Leite, P., Mesplé-Somps, S., Pasquier-Doumer, L. et Torelli, C. (2006), «Inégalités et équités en Afrique », DT/2002-11, DIAL.

Cogneau, D., Houngbedji, K. and Mesplé-Somps, S. (2016), "The Fall of the Elephant: two decades of poverty increase in Côte d'Ivoire (1988-2008)", in Arndt, C., A. McKay and F. Tarp. (eds) Growth and Poverty in Sub-Sabaran Africa. Oxford University Press

Cohen, M.A. (1974), Urban Policy and political conflict in Africa: a study of the Ivory Coast, University of Chicago Press.

Colin, J.P. et Tarouth, H. G (2017), Les élites urbaines comme nouveaux acteurs du marché foncier en Côte d’Ivoire, Géographie, économie, société, $2017 / 3$ (Vol. 19), p. 331-355.

Contamin, B. (1997), "Entreprises publiques et désengagement de l'Etat en Côte d'Ivoire », in Contamin, B. et Memel F. H. (eds), Le modèle ivoirien en questions. Crise, ajustements, recompositions. Paris: Karthala-ORSTOM, pp. 89-107.

Conte, B. (2004), Côte d'Ivoire : clientélisme, ajustement et conflit, CED Working paper, nº 101. 
Conte, B. et Sindzingre, A. (2002) "Reforms as a domestic and international process. Liberalisation and industry in Côte d'Ivoire," in Helve, H. and Nordhaug, K. (eds), Public Policy in the Age of Globalization: Responses to Environmental and Economic Crises, London, Palgrave.

Czajka, L. (2017), “Income Inequality in Côte d’Ivoire: 1985-2014”, WID.world Working paper series $\mathrm{N}^{\circ} 2017 / 8$.

Darbon D., 2012, «Classe(s) moyenne(s) : une revue de la littérature. Un concept utile pour suivre les dynamiques de l'Afrique », Afrique contemporaine ; 4, 244.

Darbon D. (2018), Turning the poor into something more inspiring: The creation of the African Middle Class controversy (à paraître).

Dozon, J.-P. (2011), Les clefs de la crise ivoirienne, Karthala, Paris.

Dubresson, Alain (1989), Villes et industrie en Côte d'Ivoire : pour une géographie de l'accumulation urbaine, Paris, Karthala.

Durand-Lasserve A., El Kadi G., Montenegro, M. A., Tribillon J.-F. (1985), « Paraitre et gérer. La résistible ascension des classes moyennes urbaines dans les pays du Tiers Monde ", Tiers-Monde, XXVI,101. pp. 41-53.

Durand-Lasserve, A. (1986), L'exclusion des pauvres dans les villes du tiers-monde, Paris, L'harmattan.

Easterly, W. 2001, The middle class consensus and economic development, Journal of Economic Growth, 6(4): 317-335.

Ehrhart, H. (2015), «Les enjeux de la nouvelle croissance ivoirienne », AFD - Macrodev n²0, Octobre 2015

Enel, C., Desgrées du Loû, A., N’Dri Yoman, T. (2015), «Les hépatites virales B et C en Côte d'Ivoire : l'urgence d'une dynamisation de la lutte », J Afr Hepato Gastroenterol, 9: 94-98

ENSETE (2013), "Enquête nationale sur la situation de l'emploi et du travail des enfants », AGEPE - Ministère de Plan et du Développement.

ENV (2015), Enquête sur les conditions de vie des ménages en Côte d'Ivoire - 2015, INS, Abidjan, Côte d'Ivoire.

ENV (2008), Enquête sur les conditions de vie des ménages en Côte d'Ivoire - 2008, INS, Abidjan, Côte d'Ivoire.

Faure, Y.-A. et Labazée, P. (eds) (2000), Petits patrons africains : entre l'assistance et le marché, Karthala, Paris.

Fauré, Y.-A. et Médard J.-F. (eds) (1982), Etat et bourgeoisie en Côte d'Ivoire, Karthala, Paris. 
FMI (2017), Perspectives Economiques Régionales - Afrique Subsaharienne : Faire redémarrer la croissance, Avril 2017, IMF, Washington.

Gastellu, J.-M. et Affou, Y. S. (1982), «Un mythe à décomposer : la Bourgeoisie de planteurs », in Fauré, Y.-A. et Médard J.-F. (1982), Etat et bourgeoisie en Côte d'Ivoire, Karthala, Paris, pp. 149-179.

Giesbert, L. and Schotte, S. (2016), Africa's New Middle Class: Fact and Fiction of Its Transformative Power, GIGA Focus | Africa | Number 1 | May 2016 | ISSN 1862-3603

Grimm, M. (2004), «A decomposition of inequality and poverty changes in the context of macroeconomic adjustment : a microsimulation study for Côte d'Ivoire », in A. F. Shorrocks et R. van der Hoeven (éd.), Growth, Inequality and Poverty. Prospects for Pro-Poor Development, Oxford, Oxford University Press, pp.197-221.

Grimm, M., C. Guénard et S. Mesplé-Somps (2002), What has happened to the Urban Population in Côte d'Ivoire Since the 1980s? An Analysis of Monetary Poverty and Deprivation Over 15 Years of Household Data, World Development, vol. 30, nº 6, p.1073-1095.

Guengant, J-P. (2014), Comment bénéficier du dividende démographique? Replacer la population au centre des trajectoires de développement de la Côte d'Ivoire, AFD-IRD-DS.

Huntington, S. (1968), Political order in changing societies, Yale University Press.

Kodila-Tedika, O., Asongu, S.A. and Kayembe, J.M. (2014), Middle class in Africa: Determinants and consequences, AGDI Working Paper, WP/14/026.

Kouassi, K. S. (2014), «Regard rétro-prospectif sur les crises ivoiriennes de 1993 à la fin de la crise postélectrorale de $2010 »$, in Viti, F. (ed), La Côte d'Ivoire, d'une crise à l'autre. Paris: L'Harmattan.

Kroeker, L. (2016), The Kenyan Middle Class and Responses to Social Security, in BoanadaFuchs, A., Gez, Y. and Waldmüller, J.M. (eds) (2016), (Re)Searching Nairobi's Middle Class, Kompreno Research Report, K0001, Kompreno, Geneva, pp.33-44.

Le Pape, M. (1997), L'énergie sociale à Abidjan: économie politique de la ville en Afrique noire, 1930-1945, Karthala, Paris.

Le Quentrec, E. (2017), Le digital au service de l'emploi informel en Afrique, Blog de la Recherche - Orange, https://recherche.orange.com/le-digital-au-service-de-lemploi-informel-enafrique/

Lezou, C.P. (2006), Le système ivoirien de protection sociale et la réalité du droit à la santé, Mémoire de maîtrise, CERAP

Lipset, S.M. (1959), Some social requisites of democracy: Economic development and political legitimacy, American Political Science Review, 53(1): 69-105. 
Loayza, N., Rigolini, J., Llorente, G. (2012), Do middle classes bring institutional reforms?, Economics Letters, 116(3): 440-444.

Loucou, J.-N. (2012), La Côte d'Ivoire Coloniale, Editions FHB \& Editions du CERAP, Abidjan (réédition 2016).

Losch, B. (1997), "A la recherche du chaînon manquant. Pour une lecture renouvelée de l'économie de plantation ivoirienne ", in in Contamin, B. et Memel F. H. (eds), Le modèle ivoirien en questions. Crise, ajustements, recompositions. Paris: Karthala-ORSTOM, pp. 205-230.

Marie, A. (1988), Politiques urbaines et sociétés civiles : le cas africain, Revue Tiers-Monde, XXIX, 116.

Melber, H. (2015), Where and What (for) is the Middle ? African and the Middle Class(es), European Journal of Development Research, 27, pp.246-254.

Melber, H. (ed) (2016), The Rise of Africa's Middle Class, Zed Books, London, 288p.

MSHP (2008), Plan stratégique de Développement des Ressources Humaines du secteur de la santé en Côte d'Ivoire 2009-2013, 55p.

MSHP (2016), Plan National de Développement sanitaire 2016-2020, (Draft consolidé), 88p.

MSLS, (2012), Stratégie nationale de financement de la santé vers la couverture universelle, 70p.

MSLS/CPS (2008), Etude sur l'utilisation des services de santé en Côte d'Ivoire.

Ncube, M. and Lufumpa, C.L. (eds) (2015), The emerging middle class in Africa, Routledge, London.

Oga A, Samba, M, N’Guessan, B.T. et Kouadio L.P. (2011), «Réflexions sur la gratuité des soins en Côte d'Ivoire en $2011 »$, Cabiers de santé publique, 10(2) : 162 -170 D 2016-2020

OCDE (2016a), Examen multidimensionnel de la Côte d'Ivoire. Volume 1 : Évaluation initiale, Série Les voies de développement, Éditions OCDE, Paris.

OCDE (2016b), Examen multidimensionnel de la Côte d'Ivoire. Volume 2: Analyse approfondie et recommandations, Série Les voies de développement, Éditions OCDE, Paris.

OCDE (2016c), Examen multidimensionnel de la Côte d'Tvoire: Volume 3: De l'analyse à l'action, Série Les voies de développement, Éditions OCDE, Paris.

Plane, P. (1997), «La privatisation de l'électricité en Côte d'Ivoire : évaluation et interprétation des premiers résultats », Tiers Monde, Tome 38, n¹52, 1997, pp862-863

Prahalad, C.K. (2004), The Fortune at the Bottom of the Pyramid: Eradicating Poverty Through Profits, Financial Times/ Prentice Hall. 
PWC (2015), Into Africa: the continent's cities of opportunity, PwC Report, Africa Business Group, Pwc.

RGPH (2014), Recensement Général de la Population et de l'Habitat - 2014, INS, Abidjan, Côte d'Ivoire

Rousset, M. et Zoro, N. (2017), Offrir des services financiers digitaux qui ont du sens pour les utilisateurs. Etudes des pratiques et instruments de gestion financières en Côte d'Ivoire, MicroSave.

Resnick, D. (2015). The Middle Class and Democratic Consolidation in Zambia, Journal of International Development, 27(5), 693-715.

Rodrik, D. (2003), In Search of Prosperity: Analytic Country Studies on Growth, Princeton, New Jersey: Princeton University Press.

Roubaud, F. (2003), "La crise vue d'en bas à Abidjan : ethnicité, gouvernance et démocratie" Afrique contemporaine, Eté 2003, n 206.

Savina, A.M., Antoine, P., Dubresson A., Yapi D. A. (1985), « Les en-haut des en-bas et les enbas des en-haut ». Classes moyennes et urbanisation à Abidjan (Côte d'Ivoire), Tiers-Monde, XXVI, 101, 1985, pp. 55-68

Schotte, S. (2017), The Anxious and the Climbers: Ambivalent Attitudes towards Democracy among South Africa’s Middle Class, GIGA Working Paper n³04.

SICOGI (2013), « Stratégies de Production de logement pour le Grand Nombre: Expériences pratiques », présentation effectuée par CAMARA Loukimane (Directeur Général de la SICOGI) au Colloque SHELTER AFRIQUE à N'DJAMENA (TCHAD) 10-13 juin 2013, disponible à http://www.shelterafrique.org/wp-content/uploads/2013/07/Cote-divoire-presentationfrench.pdf, page consultée le 13/11/2017

Soko, C. (2011), Les entrepreneurs informels en Côte d'Ivoire. Entre l'Etat, le Marché et les circuits de financement, L'Harmattan.

Strode, M., Crawfurd, L., Dettling, S. and Schmieding, F. (2015), Jobs and the Labor Market, in Ncube, M. and Lufumpa, C.L. (eds), The Emerging Middle Class in Africa, Routledge, London, pp.82-101.

Tarouth, H. G. et Colin, J.P. (2016), Les acquisitions de terres rurales par les « cadres » en Côte d'Ivoire : premiers enseignements, Cabiers Agricultures, 2016, 25, 15005.

Thurlow, J., Resnick, D. and Ubogu, D. (2015), Matching concept with measurement: Who belongs to Africa's middle class? Journal of International Development, 27, pp.588-608.

Touré, A. (1981), La civilisation quotidienne en Côte d'Ivoire. Procès d'occidentalisation, Karthala, Paris. 
UCT-Unilever Institute of Strategic Marketing - IPSOS (2017), The African Middle Class : Who are they?, Report

Vidal, C. (1997), "Du rêve au réalisme : des citadins sans illusions, 1970-1994", dans B. Contamin et H. Memel-Fotê (dir.), Le modèle ivoirien en questions, Karthala et ORSTOM, Paris, p. 655-668.

Vidal, Claudine (1991), Sociologie des passions, Paris, Karthala.

Villegas.C.M (2012), Revolution from the Middle: Class Power, Democracy and Middle Class Narratives in the Philippines, Venezuela and Ecuador, Ph.D in sociology, Brown University, May.

Visagie, J., and Posel, D. (2013), A Reconsideration of What and Who is Middle Class in South Africa, Development Southern Africa, 30(2), 149-167.

Wiemann, J. (2015). Sharing Global Responsibility: The Role of the Middle Classes on the Way to a just and Sustainable Global Economy. European Journal of Development Research, 27(2), 205-2016.

Wietzke, B., and Sumner, A. (2014), The Political and Social Implications of the "New Middle Classes" in Developing Countries: A Literature Review and Avenues for Future Research. International Development Institute Working Paper, 2014-03. 


\section{Annexe}

Tableau $n^{\circ}$ A.1. - Principales caractéristiques des individus interviewés lors des entretiens qualitatifs « ménages »

\begin{tabular}{|c|c|c|c|c|c|c|c|c|c|}
\hline $\mathbf{N}^{\circ}$ Entretiens & Genre & Age & Lieu & $\begin{array}{c}\text { Statut } \\
\text { matrimonial }\end{array}$ & $\begin{array}{l}\text { Taille du } \\
\text { ménage }\end{array}$ & Education & CSP & $\begin{array}{c}\text { Statut dans } \\
\text { l'emploi }\end{array}$ & Groupe de classe moyenne \\
\hline Entretien $\mathbf{N}^{\circ} 1$ & M & 44 & Abidjan & Marié & 05 & Secondaire & Agriculteur & Salarié-employé & Agriculteurs informels \\
\hline Entretien $\mathbf{N}^{\circ} 2$ & M & 40 & Abidjan & Marié & 06 & Supérieure & $\begin{array}{c}\text { Employés et } \\
\text { travailleurs des }\end{array}$ & Salarié-employé & Intermédiaire privée formelle \\
\hline Entretien $\mathbf{N}^{\circ} 3$ & M & 46 & Abidjan & Marié & 08 & Supérieure & $\begin{array}{l}\text { Employés et } \\
\text { travailleurs des }\end{array}$ & Salarié-employé & Intermédiaire privée formelle \\
\hline Entretien $\mathbf{N}^{\circ} 4$ & M & 40 & Abidjan & Marié & 06 & Secondaire & $\begin{array}{l}\text { Employés et } \\
\text { travailleurs des }\end{array}$ & Salarié-employé & Intermédiaire privée formelle \\
\hline Entretien $\mathrm{N}^{\circ} 5$ & M & 49 & Abidjan & Marié & 07 & Supérieure & $\begin{array}{l}\text { Employés et } \\
\text { travailleurs des }\end{array}$ & Salarié-employé & $\begin{array}{l}\text { Dirigeants cadres et professionnels } \\
\text { intermédiaires du secteur public }\end{array}$ \\
\hline Entretien $\mathbf{N}^{\circ} 6$ & $\mathrm{~F}$ & 63 & Abidjan & Marié & 05 & Supérieure & Retraité & $\begin{array}{l}\text { Inactifs et } \\
\text { chômeurs }\end{array}$ & Retraités et inactifs \\
\hline Entretien $\mathbf{N}^{\circ} 7$ & M & 47 & Abidjan & Marié & 12 & Supérieure & Agriculteur & Salarié-employé & Agriculteurs informels \\
\hline Entretien $\mathrm{N}^{\circ} 8$ & M & 38 & Abidjan & Marié & 02 & Supérieure & $\begin{array}{l}\text { Professions } \\
\text { intermédiaires }\end{array}$ & Salarié-employé & $\begin{array}{l}\text { Dirigeants cadres et professionnels } \\
\text { intermédiaires du secteur public }\end{array}$ \\
\hline Entretien $\mathbf{N}^{\circ} 9$ & M & 32 & Abidjan & Marié & 05 & Supérieure & $\begin{array}{c}\text { Professions } \\
\text { Intermédiaires }\end{array}$ & Salarié-employé & $\begin{array}{l}\text { Dirigeants cadres et professionnels } \\
\text { intermédiaires du secteur public }\end{array}$ \\
\hline Entretien $\mathrm{N}^{\circ} 10$ & M & 40 & Abidjan & Célibataire & 05 & Supérieure & $\begin{array}{l}\text { Employés et } \\
\text { travailleurs des }\end{array}$ & Salarié-employé & $\begin{array}{l}\text { Dirigeants cadres et professionnels } \\
\text { intermédiaires du secteur public }\end{array}$ \\
\hline Entretien $N^{\circ} 11$ & M & 47 & Abidjan & Marié & 15 & Primaire & $\begin{array}{l}\text { Employés et } \\
\text { travailleurs des }\end{array}$ & Indépendant & Travailleurs de l'économie informelle \\
\hline Entretien $\mathrm{N}^{\circ} 12$ & M & 43 & Abidjan & Marié & 04 & Secondaire & $\begin{array}{l}\text { Professions } \\
\text { intermédiaires }\end{array}$ & Indépendant & Travailleurs de l'économie informelle \\
\hline Entretien $N^{\circ} 13$ & M & 45 & Abidjan & Marié & 05 & Secondaire & $\begin{array}{l}\text { Professions } \\
\text { intermédiaires }\end{array}$ & Indépendant & Travailleurs de l'économie informelle \\
\hline Entretien $N^{\circ} 14$ & M & 30 & Abidjan & Marié & 03 & Secondaire & Commerçant & Indépendant & Travailleurs de l'économie informelle \\
\hline
\end{tabular}




\begin{tabular}{|c|c|c|c|c|c|c|c|c|c|}
\hline Entretien $\mathrm{N}^{\circ} 15$ & $\mathrm{~F}$ & 63 & Abidjan & Marié & 09 & Supérieure & Retraité & $\begin{array}{l}\text { Inactifs et } \\
\text { chômeurs }\end{array}$ & Retraités et inactifs \\
\hline Entretien $N^{\circ} 16$ & $\mathrm{~F}$ & 62 & Abidjan & Divorce & 08 & Supérieure & $\begin{array}{l}\text { Employés et } \\
\text { travailleurs des }\end{array}$ & $\begin{array}{l}\text { Inactifs et } \\
\text { chômeurs }\end{array}$ & Retraités et inactifs \\
\hline Entretien $N^{\circ} 17$ & $\mathrm{M}$ & 53 & Soubré & Marié & 12 & Secondaire & Agriculteur & Indépendant & Agriculteurs informels \\
\hline Entretien $N^{\circ} 18$ & $\mathrm{M}$ & 67 & Soubré & Marié & 21 & Primaire & Agriculteur & Indépendant & Agriculteurs informels \\
\hline Entretien $\mathbf{N}^{\circ} 19$ & $\mathrm{M}$ & 31 & Soubré & Marié & 05 & Secondaire & Ouvrier & $\begin{array}{l}\text { Travailleur non } \\
\text { rémunéré }\end{array}$ & Agriculteurs informels \\
\hline Entretien $\mathrm{N}^{\circ} 20$ & M & 53 & Soubré & Marié & 20 & $\begin{array}{c}\text { Sans } \\
\text { Education } \\
\end{array}$ & Agriculteur & Indépendant & Agriculteurs informels \\
\hline Entretien $N^{\circ} 21$ & $\mathrm{M}$ & 39 & Soubré & Marié & 05 & Primaire & Ouvrier & Salarié-employé & Travailleurs de l'économie informelle \\
\hline Entretien $\mathrm{N}^{\circ} 22$ & M & 40 & Soubré & Célibataire & 01 & Supérieure & $\begin{array}{c}\text { Employés et } \\
\text { travailleurs des }\end{array}$ & Salarié-employé & Travailleurs de l'économie informelle \\
\hline Entretien $N^{\circ} 23$ & $\mathrm{M}$ & 74 & Soubré & Marié & 04 & Secondaire & Retraité & Inactif et chômeur & Retraités et inactifs \\
\hline Entretien $N^{\circ} 24$ & M & 41 & Soubré & Marié & 07 & Supérieure & $\begin{array}{c}\text { Employés et } \\
\text { travailleurs des }\end{array}$ & Indépendant & $\begin{array}{l}\text { Dirigeants cadres et professionnels } \\
\text { intermédiaires du secteur public }\end{array}$ \\
\hline Entretien $N^{\circ} 25$ & $\mathrm{M}$ & 61 & Soubré & Marié & 10 & $\begin{array}{c}\text { Sans } \\
\text { Education } \\
\end{array}$ & Agriculteur & Indépendant & Agriculteurs informels \\
\hline Entretien $N^{\circ} 26$ & $\mathrm{M}$ & 47 & Soubré & Marié & 6 & Secondaire & Agriculteur & Indépendant & Agriculteurs informels \\
\hline Entretien $\mathbf{N}^{\circ} 27$ & $\mathrm{M}$ & 41 & Abidjan & Marié & 7 & Supérieure & $\begin{array}{l}\text { Employés et } \\
\text { travailleurs des }\end{array}$ & Salarié-employé & Intermédiaire privée formelle \\
\hline Entretien $\mathrm{N}^{\circ} 28$ & $\mathrm{~F}$ & 38 & Abidjan & Célibataire & 2 & Primaire & $\begin{array}{c}\text { Employés et } \\
\text { travailleurs des }\end{array}$ & Indépendant & Travailleurs de l'économie informelle \\
\hline Entretien $N^{\circ} 29$ & $\mathrm{~F}$ & 37 & Abidjan & Marié & 4 & Supérieure & $\begin{array}{c}\text { Profession } \\
\text { intermédiaire }\end{array}$ & Salarié-employé & $\begin{array}{l}\text { Dirigeants cadres et professionnels } \\
\text { intermédiaires du secteur public }\end{array}$ \\
\hline Entretien $\mathrm{N}^{\circ} 30$ & $\mathrm{M}$ & 35 & Abidjan & Marié & 2 & Supérieure & $\begin{array}{l}\text { Employés et } \\
\text { travailleurs des }\end{array}$ & Salarié-employé & Intermédiaire privée formelle \\
\hline Entretien $\mathbf{N}^{\circ} 31$ & M & 64 & Abidjan & Marié & 6 & Supérieure & Retraité & Inactif et chômeur & Retraités et inactifs \\
\hline
\end{tabular}


Demières parutions de la collection Papiers de Recherche de l'AFD

Les Papiers de Recherche de l'AFDsont disponibles sur: https://www.afd .fr/fr/ressources

\# 2017-61 BOVARI Mc ISAAC, F. (2017), "Testing Goodwin with a Stochastic Differential Approach-The United States (1948-2017)", AFD Research Papers Series, No. 2017-61, November.

\# 2017-62 Mc ISAAC, F., D. BASTIDAS (2017), "An Input-Output Analysis: What Would a Low-Carbon Economy for Brazil Mean?", AFD Research Papers Series, No. 2017-62, December.

\# 2017-63 DAVID, A., GUILBERT, N., HINO, H., LEIBBRANDT, M., POTGIEIER, E. and M. SHIFA (2018), "Social Cohesion and inequality in South Africa", AFD Research Papers Series, No. 2018-63, J a nuary.

\# 2018-64 DE BERCEGOL, R., J. CAVÉ and A. NGUYEN THAI HUYEN (2018), "Informal Recycling vs municipal Waste Service in Asian cities: Opposition or Integration?", AFD Research Papers Series, No. 2018-64, January.

\# 2018-65 BOVARI, E., G. GIRAUD and F. Mc ISAAC (2018), "Carbon Pricing and Global Warming: A Stock-flow Consistent Macro-dynamic Approach", AFD Research Papers Series, No. 2018-65, J a nuary.

\# 2018-66 DAVID, A., N. GUILBERT, N. HAMAGUCHI, Y. HIGASH, H. HINO, M. LEIBBRANDT and M. SHIFA (2018), "Spa tial poverty and inequality in South Africa: A municipality level analysis", AFD Research Papers Series, No. 2018-66, J a nuary.

\# 2018-67 CLÉMENT, M., Y.-A. Faure, J.-P. BERROU, F. COMBARNOUS, D. DARBON et E. ROUGIER (2018), “Anatomie de la classe moyenne brésilienne: identification, caractérisation et implications pour les politiques publiques", Pa piers de recherche AFD, $n^{\circ}$ 2018-67, J uin.

\# 2018-68 MAUREL, M. (2018), “Régionalisme en Afrique : coûts de transport «soft » and «hard »", Papiers de Recherche AFD, n 2018-68, J uin.

\# 2018-69 MILUSHEVA, S., E. zU ERBACH-SCHO ENBERG, L. BENGTSSON, E. WETTER and A. TATEM (2017), "Understanding the Relationship between Short and Long Term Mobility", AFD Research Papers Series, No. 2018-69, June.

\# 2018-70 DE NADAI, M., E. LETOUŹ, M.C. GONZÁLEZ and B. LEPRI (2017), "Characterizing and analyzing urban dynamics in Bogota", AFD Research Paper Series, No. 2018-70, J une. 